\title{
NUMERICAL ANALYSIS OF A REYNOLDS STRESS MODEL FOR TURBULENT MIXING: THE ONE-DIMENSIONAL CASE
}

\author{
Xavier Blanc $^{1} \oplus$, Charles Colavolpe ${ }^{2}$, Roland Duclous $^{2, *} \odot$, Jérôme Griffond ${ }^{2}$ \\ AND OLIVIER SOULARD ${ }^{2}$
}

\begin{abstract}
A mixed hyperbolic-parabolic, non conservative, Reynolds Stress Model (RSM), is studied. It is based on an underlying set of Langevin equations, and allows to describe turbulent mixing, including transient demixing effects as well as incomplete mixing. Its mathematical structure is analysed, and specific regimes, related to acoustic-like, Riemann-type, or self-similar solutions, are identified. A second-order accurate numerical scheme is proposed in arbitrary curvilinear geometry. Its accuracy and convergence behaviour are tested by comparison with analytical solutions in the different regimes. The numerical scheme can be generalized to multi-dimensional configurations, with potentially cylindrical symmetry, on unstructured meshes.
\end{abstract}

Mathematics Subject Classification. 65M12,65M22, 76F25.

Received May 9, 2020. Accepted July 13, 2021.

\section{INTRODUCTION}

Turbulent mixing at fluid interfaces plays an important role in a wide variety of domains, ranging from the study of astrophysical objects like supernovae, to engineering applications like Inertial Confinement Fusion (ICF). Depending on the configuration, turbulent mixing can be triggered by different instabilities, among which the Rayleigh-Taylor and Richtmyer-Meshkov instabilities. Reynolds Stress Models (RSMs), presented in Section 2, are common tools to predict the main effects and evolution of such fully developed turbulence. RSMs are one-point statistical models, which rely on a local-in-space decomposition of instantaneous fields into a mean and a turbulent fluctuating field. Still, they do not compute the fluctuating fields, but rather compute their double correlations. Among existing RSMs, the BHR [2] and GSG [19, 20] models are widely used for engineering purposes related to variable-density turbulence and mixing at interfaces. They rely on a set of evolution equations for the covariances of velocity and specific volume (or other equivalent covariances, in the case of the BHR model). However, the mass fraction fluxes, which are defined as the covariances between velocity and mass fractions, are algebraically closed, and expressed in terms of the evolved variables of the model. Hence a first gradient closure is used, which implies that the material mass fractions evolve according to a nonlinear parabolic equation. This modelling is firmly established in the self-similar regime, assuming turbulent spectra

Keywords and phrases. Turbulence, Reynolds Stress Model, incomplete mixing, demixing, hyperbolic system.

1 Université de Paris, Sorbonne Université, CNRS, Laboratoire Jacques Louis Lions, F-75013 Paris, France.

2 CEA, DAM, DIF, F-91297 Arpajon, France.

*Corresponding authour: roland.duclous@cea.fr

(C) The authors. Published by EDP Sciences, SMAI 2021

This is an Open Access article distributed under the terms of the Creative Commons Attribution License (https://creativecommons.org/licenses/by/4.0), which permits unrestricted use, distribution, and reproduction in any medium, provided the original work is properly cited. 
at equilibrium, but it lacks some important phenomenology, as mentioned in [37,38]. More precisely, countergradient turbulent transport, with transient demixing effects, and incomplete mixing, with segregation effects, are not described. Yet, they are likely to appear in many configurations of interest, for instance just after a shock wave crosses a Turbulent Mixing Zone (TMZ) from the heavy-fluid side to the light-fluid side [8]. In order to take into account these effects, the closure on mass fraction fluxes should be postponed at a higher level by adding evolution equations for the mass fraction fluxes and covariances. This yields a coherent second-order model (in a sense that will be precised in Sect. 2).

In doing so, the nature of the model changes. The conservative parabolic equation for the material mass fractions is superseded by a non-conservative, mixed hyperbolic-parabolic subsystem. Physically relevant solutions rely on both hyperbolic and parabolic parts of the model, with comparable magnitude (see the analytical solutions of Sect. 3.2.2), at least when the characteristic time of the external forcing by the mean flow is not too small compared to the turbulent one. However, the regularizing closure may become unphysical when strong accelerations occur, for instance when a shock wave crosses a TMZ. In that case, the parabolic terms imply a supersonic transport of information from the shocked flow to the flow upstream from the shock front. This violation of a basic physical principle leads to a "modelling" instability as shown in [36]. Such failures of the closures must be cured through limitations which reduce or even discard the regularizing terms.

The purpose of this work is to propose a numerical scheme that can be applied to the hyperbolic subsystem, appart from the regularizing terms. The proposed scheme falls in the class of Godunov-type methods, that rely on a solution of the Riemann problem. While they seem appropriate in our case, at first sight (an exact solution of the Riemann problem is known), they should be employed with care and adapted. The proposed adaptation is rather specific to our turbulence RSM, in the sense that some non-conservative products, which are not defined, even in the weak sense, for the Riemann problem, require an appropriate treatment. Other adapations were introduced to enforce discrete invariant domains (realizability), entropy stability and stationary states, in 1D cuvilinear coordinates. These latter accuracy and stability requirements are used by a large class of numerical schemes devoted to non-conservative hyperbolic systems [7,41]. In the context of other turbulence RSM, discrete entropy and realizability have already been discussed $[5,6]$, but from a different point of view (with emphasis on non-conservative products in presence of shocks). Our approximate Godunov scheme is extended to second-order accuracy, and can be generalized to multi-dimensional and non-planar configurations, on unstructured meshes. Little will be said here on the numerical treatment of parabolic operators, having a compact diffusion support, since the turbulent diffusion terms do not exhibit any particular stiffness. This is not the main concern of this work.

The article is organized as follows. In Section 2, the RSM is presented, its origins are detailed. In Section 3, a mathematical analysis of the hyperbolic-parabolic subsystem is proposed. Filiation with other RSMs is pointed out. The mathematical analysis supports a numerical analysis in 1D curvilinear coordinates, in Section 4. A first-order scheme is proposed, which constitutes a basis for a subsequent second-order extension in space and time. A special attention is paid to the simplicity of the algorithm and to stability at each step, having in view the generalization to an unstructured multi-D scheme. In Section 5, we investigate, in a series of numerical tests, the response of the proposed scheme with respect to the variety of possible regimes allowed by the RSM.

\section{Model}

The model studied in the present article is a building block of a larger-purpose turbulence model intended to predict turbulent mixing in multi-species compressible flows subject to strong accelerations or decelerations and shock crossing as encountered in ICF applications [24] or shock tube experiments [8,29]. In its previous form $[20,21]$, this model focuses on the effect of pressure and velocity gradients but employs a simple approach for the species mass fractions. Its present extension introduces a more general treatment of concentrations leading to a more complex system of equations. Since the analysis of the full coupled model is a difficult task, this article makes a first step by considering the simplified but physically meaningful situations of freely evolving turbulent mixing zones where the mean pressure and velocity gradients can be discarded. 
To be more specific, the model under consideration here pertains to the class of the Reynolds-averaged Navier-Stokes (RANS) turbulence models. RANS models rely on the decomposition of each flow variable $q$ into a mean $\bar{q}$ and a fluctuating part $q^{\prime}=q-\bar{q}$ with respect to a well-suited Reynolds average ${ }^{1}$, for instance an ensemble average (as defined in [16], Appendix 1) as in the present case. Writing the equations for the mean values $\bar{q}$ of the instantaneous variables involves unknown correlations which require to be either modelled from known quantities or evolved from their own equation which anew bring forth correlations of even larger order. This decomposition procedure can be illustrated with the instantaneous equation for the conservation of the mass of a material of index $\alpha$. The latter reads $\frac{\partial}{\partial t}\left(\rho Y_{\alpha}\right)+\nabla \cdot\left(\rho Y_{\alpha} \mathbf{U}\right)=0$ with $\rho$ the density, $Y_{\alpha}$ the mass fraction (i.e. mass of material $\alpha$ per unit mass of the mixture, made of $N_{m}$ materials, such that $\sum_{\alpha=1}^{N_{m}} Y_{\alpha}=1$ ) and $\mathbf{U}$ the velocity. When working with conservation equations, it is convenient to use Favre (i.e. mass-weighted) averages together with Reynolds averages. For any quantity $q$, the Favre average is denoted $\widetilde{q}$ and is related to the Reynolds average, $\bar{q}$, according to $\widetilde{q}=\overline{\rho q} / \bar{\rho}$, with $\rho$ the instantaneous density. The Favre fluctuation is then denoted with $q^{\prime \prime}=q-\widetilde{q}$. Summing over the $\alpha$ indices, then averaging the mass conservation equation leads to $\frac{\partial \bar{\rho}}{\partial t}+\nabla \cdot(\bar{\rho} \tilde{\mathbf{U}})=0$ and does not involve any unclosed correlation, when the Favre average of the velocity $\tilde{\mathbf{U}}$ is used instead of its Reynolds averaged counter-part. On the opposite, the RANS procedure leads to the following unclosed equation for the averaged mass fraction $\bar{\rho} \frac{\partial \tilde{Y}_{\alpha}}{\partial t}+\bar{\rho} \tilde{\mathbf{U}} \cdot \nabla \tilde{Y}_{\alpha}+\nabla \cdot\left(\bar{\rho} \widetilde{Y_{\alpha}^{\prime \prime} \mathbf{u}^{\prime \prime}}\right)=0$ where the turbulent scalar flux $\widetilde{Y_{\alpha}^{\prime \prime} \mathbf{u}^{\prime \prime}}$ is an unknown quantity. A simple approach consists in writing a closure based on phenomenological considerations $\widehat{Y_{\alpha}^{\prime \prime} \mathbf{u}^{\prime \prime}}=-\nu_{T} \nabla \tilde{Y}_{\alpha}$ where the so-called turbulent diffusivity $\nu_{T} \geq 0$ has to be expressed from known correlations. Such a formula, reminiscent of the Fickian approximation for diffusion processes, is referred to as a first gradient closure. A more general approach consists in writing an equation for the flux $\overline{Y_{\alpha}^{\prime \prime} \mathbf{u}^{\prime \prime}}$ by combining equations for $Y_{\alpha}^{\prime \prime}$ and for $\mathbf{u}^{\prime \prime}$ but this implies that the unknown third order correlation $Y_{\alpha}^{\prime \prime} \mathbf{u}^{\prime \prime} \otimes \mathbf{u}^{\prime \prime}$ requires a closure. Assumptions and approximations allowing to retrieve the gradient closure of correlations from their unclosed equations can be found in [35], which serves as a guide for defining the turbulent diffusivity and having an idea of the domain of validity of the simple closure. RANS models are called first-order turbulence models when closures are applied to second-order correlations, whereas they are second-order turbulence models when only third-order correlations are closed, while second-order correlations are kept as unknown of the system. The second-order correlation tensor of the fluctuating velocity, $\widetilde{\mathbf{u}^{\prime \prime} \otimes \mathbf{u}^{\prime \prime}}$, is an important variable of RANS models. It is referred to as the Reynolds stress tensor. Its half trace $\tilde{k}=\frac{1}{2} \operatorname{tr}\left(\widetilde{\mathbf{u}^{\prime \prime} \otimes \mathbf{u}^{\prime \prime}}\right)$ is the turbulent kinetic energy. The rate of molecular destruction of $\tilde{k}$ is described by the variable $\tilde{\varepsilon}$. The ratio $\tilde{\omega}=\tilde{\varepsilon} / \tilde{k}$ is a characteristic turbulent frequency. Many models decompose the Reynolds stress tensor into an isotropic and a deviatoric part and use a closure for the latter, thereby needing only one equation for $\tilde{k}$, rather than equations for each component of the tensor. On the opposite, models which evolve the full Reynolds stress tensor are called Reynolds stress models (RSM). The present model is of that kind.

Some RSMs can be obtained following two distinct but coherent derivation routes. The first route is determined by the procedure described above. It starts from the hydrodynamics equations for the instantaneous fields, introduces a decomposition into a mean and a turbulent fluctuating component, isolates and averages the equations for the products of fluctuations and finally models the unclosed correlations at second order. The second route relies on an underlying model, which directly computes the Probability Density Function (PDF) of the fluctuating fields [31]. This ensures that the tensor built from the double correlations of the fluctuating fields is semi definite positive. Hence, the resulting RSM stands as realizable. It is this second route that is followed to present the RSM under study.

In order to give more insights into the origins of the RSM of interest here, we introduce its underlying PDF model. The RSM shares some important features with RSM equations presented in [39] (see the turbulent flux Eq. (223), terms I to IV), while the underlying PDF model is adapted from [30,32]. It is able to account for a number of important identified mechanisms that occur, for instance, but not only, in turbulence induced by the Richtmyer-Meshkov instability. For the sake of simplicity, the underlying PDF model will be described for

\footnotetext{
${ }^{1}$ A Reynolds average obeys a set of rules named by Hinze [23] after the pioneer of turbulence studies O. Reynolds.
} 
this particular type of flows. Thus, before presenting the PDF model, we will first give a few details about Richtmyer-Meshkov turbulent mixing zones. They result from the impulsional energy deposit by a shock wave crossing an interface between two fluids. The unavoidable small scale perturbations of this interface grow in time, proportionally to the Atwood number, $A_{t}=\frac{\rho_{h}-\rho_{l}}{\rho_{h}+\rho_{l}}$, which compares the densities, $\rho_{l}$ and $\rho_{h}$, of the lighter and heavier fluids, respectively. The instability ultimately reaches a self-similar, fully turbulent state. Before reaching such a state, compressibility effects progressively fade: the shock gets far from the mixing zone and the Boussinesq approximation becomes valid $[37,38,44]$. The mean acceleration, mean pressure gradient, $\nabla \bar{P}$, and mean velocity gradient, $\nabla \widetilde{\mathbf{U}}$, become negligible. The only turbulence production comes from the mean mass fraction gradients, which drive, through the fluctuating fields, both turbulent mass fluxes and a modification of the mixing heterogeneity. This low compressibility limit regime embeds a variety of non-trivial aspects of fully developed turbulence: fluctuating velocity field showing incomplete return to isotropy, transient demixing effects, and incomplete mixing (defined by $\widehat{Y_{\alpha}^{\prime \prime 2}} \neq 0$ ) between materials $[37,38]$.

To model this type of flows, it has been shown that the Langevin model got satisfactory results [30, 32], notwithstanding the issue of anisotropy, that will be left aside, for the sake of simplicity. To deal with mixing, we also propose to use Langevin models, and their corresponding PDF model. Namely, a Langevin model inspired from the Interaction Exchange with the Mean (IEM) model for the instantaneous mass fractions [14], together with a Simplified Langevin Model (SLM) [31] for the fluctuating velocities, with only dissipation and complete redistribution (i.e. complete return to isotropy) terms. The IEM model has been enriched with a Brownian noise, in order to include, by construction, a class of self-similar solutions in the subsequent RSM. Self-similarity is indeed an important, generic feature of a wide class of fully developed turbulent regimes, that cannot be disregarded. Even though it does not allow the respect of the mass fraction boundedness, this model is only meant to be used as a basis for a RSM. For this purpose, this inherent defect of the Langevin model applied to a turbulent field is not prohibitive. This bare Langevin model can be enriched with mean acceleration and compressibility effects, for instance to account for the large scale interaction with a shock [20]. Such an extension will not be considered in this work and we only focus on these reduced Langevin models.

The flow under consideration involves $N_{m}$ materials, also referred to as "species". For each material $\alpha \in\left[1, N_{m}\right]$ composing the fluid, the mass fraction is defined as $Y_{\alpha}=\rho_{\alpha} / \sum_{\beta=1}^{N_{m}} \rho_{\beta}$, where $\rho_{\alpha}$ is the local mass of material $\alpha$ per unit volume, so that $\rho=\sum_{\beta=1}^{N_{m}} \rho_{\beta}$ is the fluid density. Each material is itself composed of different constituents among the $N_{c}$ ones over the whole set of materials. The mass fraction of constituent $i$, in material $\alpha$, is defined as $Y_{\alpha, i}=\rho_{\alpha, i} / \rho$, where $\rho_{\alpha, i}$ is the local mass of constituent $i$ belonging to the material $\alpha$, per unit volume. As an illustration, ICF capsules [24] involve materials such as the filling gas and solid shells, whereas the deuterium and tritium ions which should fusion are constituents that can be present in different materials. Mixing between materials can be triggered by molecular or turbulent diffusion. The turbulent material mass fraction flux of each material $\alpha, \widehat{Y_{\alpha}^{\prime \prime} \mathbf{u}^{\prime \prime}}$, is a cornerstone to the turbulent mixing modeling. It can be obtained by integration of the Favre PDF, $\widetilde{f}$, over the fluctuating field $\mathbf{q}^{\prime \prime}=\left(\mathbf{u}^{\prime \prime}, Y_{\alpha}^{\prime \prime}, Y_{\beta}^{\prime \prime}, \cdots\right)$ : $q_{i}^{\prime \prime} q_{j}^{\prime \prime}=\int \mathrm{d} \mathbf{q}^{\prime \prime} q_{i}^{\prime \prime} q_{j}^{\prime \prime} \widetilde{f}\left(\mathbf{q}^{\prime \prime}\right)=\mathbb{X}_{i j}$. The so-called correlation tensor, $\mathbb{X}$, writes

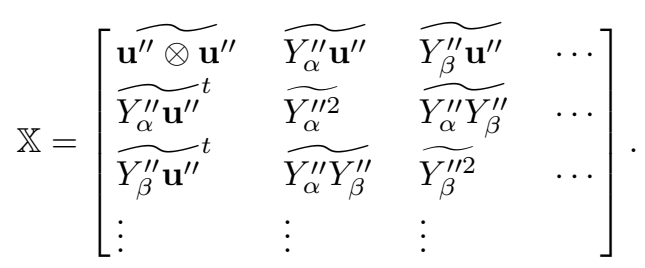

The Favre PDF satisfies the following equation

$$
\begin{aligned}
\frac{\partial}{\partial t}\left[\bar{\rho} \widetilde{f}\left(\mathbf{q}^{\prime \prime}\right)\right]+\nabla \cdot\left[\bar{\rho}\left(\widetilde{\mathbf{U}}+\mathbf{u}^{\prime \prime}\right) \widetilde{f}\left(\mathbf{q}^{\prime \prime}\right)\right]+\nabla_{q^{\prime \prime}} \cdot\left[\widetilde{f}\left(\mathbf{q}^{\prime \prime}\right) \nabla \cdot\left(\bar{\rho} \mathbf{u}^{\prime \prime} \otimes \mathbf{q}^{\prime \prime}\right)\right]= & -\nabla_{q^{\prime \prime}} \cdot\left[\bar{\rho} \widetilde{f}\left(\mathbf{q}^{\prime \prime}\right) \mathbb{G} \mathbf{q}^{\prime \prime}\right] \\
& +\frac{1}{2} \mathbb{H} \mathbb{H} \mathbb{H}^{t}: \nabla_{q^{\prime \prime}}^{2}\left[\bar{\rho} \widetilde{f}\left(\mathbf{q}^{\prime \prime}\right)\right] .
\end{aligned}
$$


The left-hand side gathers terms related to turbulent transport, that arise from the decomposition of the NavierStokes equations into the mean and fluctuating components. The procedure that leads to these terms is described in [32], for instance. The right-hand side is a Fokker-Planck model. It is commonly used in turbulence modelling [32], as it can handle with Gaussian PDF with appropriate mean and variance of fluctuating quantities. The coefficients $\mathbb{G}$ and $\mathbb{H}$ are defined by

$$
\mathbb{G} \equiv\left[\begin{array}{ll}
G_{u u} \mathbb{I}_{3} & \mathbf{0} \\
G_{p} & G_{d} \mathbb{I}_{N_{m}}
\end{array}\right], \quad \mathbb{H} \equiv\left[\begin{array}{ll}
H_{u} \mathbb{I}_{3} & \mathbf{0} \\
\mathbf{0} & \mathbb{H}_{b}
\end{array}\right]
$$

where the diagonal components of $\mathbb{G}$, that are $G_{u u}$ and $G_{d}$, model the fluctuation dissipation - or return to the mean -, while the contribution $\mathbb{H} \mathbb{H}^{t}$ accounts for the dispersion from the mean due to the turbulent motion. $\mathbb{I}_{n}$ is the identity matrix of dimension $n$. The coefficients that govern the fluctuating velocity dissipation and dispersion are $G_{u u}=-\frac{1}{2} C_{1} \widetilde{\omega}$ and $H_{u}^{2}=\frac{2}{3}\left(C_{1}-1\right) \widetilde{\omega} \widetilde{k}$, respectively. Here, $C_{1}>1$ is a positive constant related to the redistribution processes in the fluctuating velocity components, and determines the isotropization rate of the Reynolds stress tensor. On the other hand, the coefficients that govern the fluctuating material mass fraction dissipation and dispersion are $G_{d} \equiv-\frac{C_{\tau}}{2} \widetilde{\omega}$ and $\mathbb{H}_{b}$, respectively. The off-diagonal coefficient $G_{p} \equiv\left(\begin{array}{c}-\left[\nabla \tilde{Y}_{\alpha}\right]^{t} \\ -\left[\nabla \widetilde{Y}_{\beta}\right]^{t} \\ \vdots\end{array}\right)$ accounts for turbulence production by the material mass fraction gradients. The tensorial

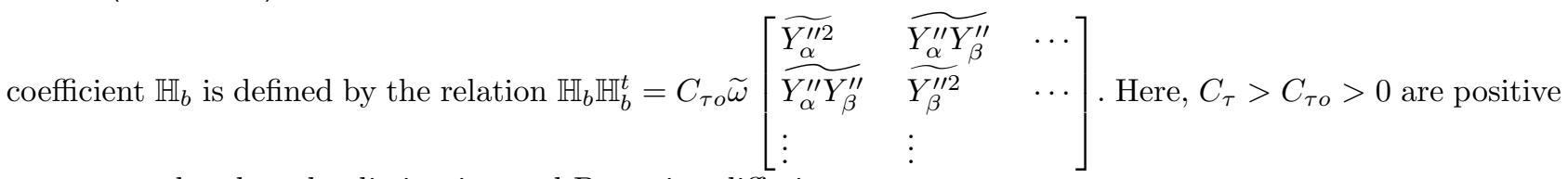
constants related to the dissipation and Brownian diffusion processes.

Remark 2.1. Consider the mass fraction and velocity fluctuations, $Y_{\alpha}^{\prime \prime}$ and $\mathbf{u}^{\prime \prime}=\left(u_{x}^{\prime \prime}, u_{y}^{\prime \prime}, u_{z}^{\prime \prime}\right)^{t}$, be solution of

$$
\begin{aligned}
\mathrm{D}_{\mathrm{t}} Y_{\alpha}^{\prime \prime} & =-\mathbf{u}^{\prime \prime} \cdot \nabla \widetilde{Y}_{\alpha}+\frac{1}{\bar{\rho}} \nabla \cdot\left(\widetilde{\rho} \widetilde{Y_{\alpha}^{\prime \prime} \mathbf{u}^{\prime \prime}}\right)-\frac{C_{\tau}}{2} \widetilde{\omega} Y_{\alpha}^{\prime \prime}+\sqrt{C_{\tau o} \widetilde{\omega}} \sum_{\beta=1}^{N_{m}} H_{\alpha \beta} \dot{W}_{\beta}, \\
\mathrm{D}_{t} \mathbf{u}^{\prime \prime} & =\frac{1}{\bar{\rho}} \nabla \cdot\left(\bar{\rho} \widetilde{\mathbf{u}^{\prime \prime} \otimes \mathbf{u}^{\prime \prime}}\right)+G_{u u} \mathbf{u}^{\prime \prime}+\sqrt{H_{u}^{2}} \dot{\mathbf{W}}_{u}, \\
\mathrm{D}_{t} \mathbf{x} & =\widetilde{\mathbf{U}}+\mathbf{u}^{\prime \prime},
\end{aligned}
$$

where $\mathbf{x}$ is the position of a Lagrangian fluid particle. Let $f$ be the corresponding Eulerian PDF, as defined in $[30,32]$. Then, from $[30,32]$, it can be shown formally that $f$ is solution to the Fokker-Planck equation (2.1). Here, $W_{\beta}$ and $\mathbf{W}_{u}$ are independent Brownian noises, and $H_{\alpha \beta}$ is implicitely defined by the relation $\sum_{\gamma=1}^{N_{m}} H_{\alpha \gamma} H_{\beta \gamma}=\widetilde{Y_{\alpha}^{\prime \prime} Y_{\beta}^{\prime \prime}}, D_{t}$ is the Lagrangian time derivative, $\mathbf{x}$ the position of a fluid particle. The Langevin equations (2.3), for the Favre velocity fluctuations, $\mathbf{u}^{\prime \prime}$, is the SLM (12.109) from [32]. The first term in the right-hand side is a turbulent pressure contribution, that arises from the decomposition between the mean and fluctuating components of the Navier-Stokes equations. Equation (2.3) can also be viewed as a simplification of equation (B3) of [20], for the limited scope of the present framework without pressure nor velocity gradients. The Langevin equation (2.2) is a multi-species generalization of the Langevin model (5.52) of [30] (note that Eq. (2.2) is expressed as a Langevin equation for a fluctuating quantity, while Eq. (5.52) of [30] is expressed as a Langevin equation for an instantaneous quantity. The passage from one to another can be made using the equation for the averaged material mass fraction, $\tilde{Y}_{\alpha}$ ). It satisfies the mass conservation property $\sum_{\alpha=1}^{N_{m}} Y_{\alpha}^{\prime \prime}=0$. The two first terms in the right-hand side of equation (2.2) also arise from the decomposition between the mean and fluctuating components of the Navier-Stokes equations. 
To many uses, the instantaneous mass fractions $Y_{\alpha}$ are attached to a flow made of well-mixed constituents. In this work, it is assumed that there is no separation effect between constituents inside a material. Thus, the instantaneous mass fractions of the material constituents satisfy the relation

$$
Y_{\alpha, i}=\frac{\widetilde{Y}_{\alpha, i}}{\widetilde{Y}_{\alpha}} Y_{\alpha}, \quad \forall \alpha \in\left[1, N_{m}\right], \forall i \in\left[1, N_{c}\right]
$$

This amounts to a so-called "carrying material" approximation.

The equations for the second-order correlations can be obtained by integrating the PDF equation (2.1). A compact tensorial formulation of the evolution equations for the second-order correlations can be written as

$$
\frac{\mathrm{d}}{\mathrm{d} t} \mathbb{X}-\nabla \cdot \mathcal{F}^{c}=\mathbb{G} \mathbb{X}+\mathbb{X} \mathbb{G}^{t}+\mathbb{H} \mathbb{H}^{t},
$$

where $\frac{\mathrm{d}}{\mathrm{d} t}[\cdot] \equiv \frac{\partial}{\partial t}[\cdot]+\widetilde{\mathbf{U}} \cdot \nabla[\cdot]$. The triple correlation flux, yet to be closed, is denoted by $\mathcal{F}^{c}$.

The latter tensorial expression may also be developed component-wise, say for a material with index $\alpha$, having a constituent with index $i$, in presence of another material with index $\beta$. Also, the equations for the mean material mass fractions and their constituent mass fractions can be deduced from the Favre average of the instantaneous equation $\frac{\partial}{\partial t}\left(\rho Y_{\alpha}\right)+\nabla \cdot\left(\rho Y_{\alpha} \mathbf{U}\right)=0$, and from equation (2.5). This leads to the following system

$$
\begin{aligned}
& \frac{\mathrm{d}}{\mathrm{d} t} \bar{\rho}=0, \\
& \bar{\rho} \frac{\mathrm{d}}{\mathrm{d} t} \widetilde{Y}_{\alpha}+\nabla \cdot\left(\widetilde{\rho} \widetilde{Y_{\alpha}^{\prime \prime} \mathbf{u}^{\prime \prime}}\right)=0, \\
& \bar{\rho} \frac{\mathrm{d}}{\mathrm{d} t} \widetilde{Y}_{\alpha, i}+\nabla \cdot\left(\widetilde{\rho} \widetilde{Y_{\alpha}^{\prime \prime} \mathbf{u}^{\prime \prime}} \frac{\widetilde{Y}_{\alpha, i}}{\widetilde{Y}_{\alpha}}\right)=0, \\
& \frac{\mathrm{d}}{\mathrm{d} t} \widetilde{Y_{\alpha}^{\prime \prime} \mathbf{u}^{\prime \prime}}+\widetilde{\mathbf{u}^{\prime \prime} \otimes \mathbf{u}^{\prime \prime}} \nabla \widetilde{Y}_{\alpha}+\frac{1}{\bar{\rho}} \nabla \cdot\left(\bar{\rho} Y_{\alpha}^{\prime \prime} \widetilde{\mathbf{u}^{\prime \prime} \otimes} \mathbf{u}^{\prime \prime}\right)^{c}=-\frac{1}{2}\left(C_{\tau}+C_{1}\right) \widetilde{\omega} \widetilde{Y_{\alpha}^{\prime \prime} \mathbf{u}^{\prime \prime}},
\end{aligned}
$$

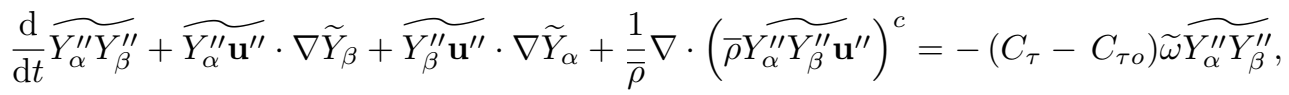

$$
\begin{aligned}
& \frac{\mathrm{d}}{\mathrm{d} t} \widetilde{\mathbf{u}^{\prime \prime} \otimes \mathbf{u}^{\prime \prime}}+\frac{1}{\bar{\rho}} \nabla \cdot\left(\bar{\rho} \mathbf{u}^{\prime \prime} \otimes \widetilde{\mathbf{u}^{\prime \prime} \otimes} \mathbf{u}^{\prime \prime}\right)^{c}=-\frac{2}{3} \widetilde{\omega} \widetilde{k} \mathbb{I}-C_{1} \widetilde{\omega}\left[\widetilde{\mathbf{u}^{\prime \prime} \otimes \mathbf{u}^{\prime \prime}}-\frac{2}{3} \widetilde{k} \mathbb{I}\right] .
\end{aligned}
$$

Remark 2.2. In these RSM equations, the material time derivative, $\frac{\mathrm{d}}{\mathrm{d} t}[\cdot] \equiv \frac{\partial}{\partial t}[\cdot]+\widetilde{\mathbf{U}} \cdot \nabla[\cdot]$, has been used. In an Arbitrary Lagrangian Eulerian (ALE) formulation, a moving volume control of arbitrary velocity $\dot{\mathbf{X}}$ is introduced. The time derivative should then be rewritten as $\frac{\mathrm{d}}{\mathrm{d} t}[\cdot]=\frac{\partial}{\partial t}[\cdot]+(\widetilde{\mathbf{U}}-\dot{\mathbf{X}}) \cdot \nabla[\cdot]$, different from the material derivative.

Finally, an equation for the turbulent mean dissipation rate, $\widetilde{\varepsilon}$, completes the system [15]

$$
\bar{\rho} \frac{\mathrm{d}}{\mathrm{d} t} \widetilde{\varepsilon}+\nabla \cdot\left(\bar{\rho} \widetilde{\varepsilon \mathbf{u}^{\prime \prime}}\right)^{c}=-C_{\varepsilon 2} \bar{\rho} \widetilde{\omega} \widetilde{\varepsilon},
$$

where $C_{\varepsilon 2}>1$ is introduced as a dissipation constant.

The last term in the right-hand side of equation (2.12) is a redistribution term among the components of the Reynolds stress tensor, which reflects its isotropization. All other terms in the right-hand side of equations (2.10)-(2.13) are interpreted as dissipation terms, as they carry a minus sign.

In order to complete the modelling at second-order, a closure on the triple correlations needs to be introduced. These are closed with a first gradient model. Note that such modelling relies on a presumed asymptotic regime 
where external sollicitations can be discarded, which is valid at long time scales. Such physical considerations hold for both first-order and second-order RSMs [34]. The second-order closure reads

$$
\begin{aligned}
& \left(\bar{\rho} Y_{\alpha}^{\prime \prime} \widetilde{\mathbf{u}^{\prime \prime} \otimes} \mathbf{u}^{\prime \prime}\right)^{c}=-C_{d} \bar{\rho} \frac{\widetilde{\mathbf{u}^{\prime \prime} \otimes \mathbf{u}^{\prime \prime}}}{\widetilde{\omega}} \nabla \widetilde{Y_{\alpha}^{\prime \prime} \mathbf{u}^{\prime \prime}}, \\
& \left(\bar{\rho} \widetilde{Y_{\alpha}^{\prime \prime} Y_{\beta}^{\prime \prime} \mathbf{u}^{\prime \prime}}\right)^{c}=-C_{d} \bar{\rho} \frac{\widetilde{\mathbf{u}^{\prime \prime} \otimes \mathbf{u}^{\prime \prime}}}{\widetilde{\omega}} \nabla \widetilde{Y_{\alpha}^{\prime \prime} Y_{\beta}^{\prime \prime}}, \\
& \left(\bar{\rho} \mathbf{u}^{\prime \prime} \widetilde{\otimes \mathbf{u}^{\prime \prime} \otimes} \mathbf{u}^{\prime \prime}\right)^{c}=-C_{d} \bar{\rho} \frac{\widetilde{\mathbf{u}^{\prime \prime} \otimes \mathbf{u}^{\prime \prime}}}{\widetilde{\omega}} \nabla \widetilde{\mathbf{u}^{\prime \prime} \otimes \mathbf{u}^{\prime \prime}}, \\
& \left(\bar{\rho} \widetilde{\mathbf{u}^{\prime \prime}}\right)^{c}=-C_{\varepsilon} \bar{\rho} \frac{\widetilde{\mathbf{u}^{\prime \prime} \otimes \mathbf{u}^{\prime \prime}}}{\widetilde{\omega}} \nabla \widetilde{\varepsilon},
\end{aligned}
$$

where $C_{d}>0$ and $C_{\varepsilon}>0$ are positive diffusion constants related to the transport processes.

The model (2.7)-(2.13), supplemented with these second-order closures, may be viewed either as an asymptotic limit, in the so-called Boussinesq regime, with constant-in-time mean density $\bar{\rho}$ (Eq. (2.7)), or simply as a sub-system of larger purpose RSMs dedicated to time-dependent, variable-density, compressible flows (by considering a general Reynolds averaged Navier-Stokes equations, that embeds the present RSM, without Eq. (2.7)). It is intended to cure several flaws of these RSMs $[2,19,20,28]$. First, the model becomes fully second-order. This ensures a better consistency between the various fields, since only triple correlations are closed. In particular, the turbulent material mass fraction fluxes, $\widehat{Y_{\alpha}^{\prime \prime} \mathbf{u}^{\prime \prime}}$, are now computed by equation (2.10), instead of being closed, in equation (2.8), with the first-order closure

$$
\widetilde{\rho} \widetilde{Y_{\alpha}^{\prime \prime} \mathbf{u}^{\prime \prime}}=-C_{d} \bar{\rho} \frac{\widetilde{\mathbf{u}^{\prime \prime} \otimes \mathbf{u}^{\prime \prime}}}{\widetilde{\omega}} \nabla \widetilde{Y}_{\alpha},
$$

as was classically done in $[2,19]$. Hence, demixing and counter-gradient fluxes (i.e. fluxes that are not oriented according to the direction imposed by the minus sign in Eq. (2.14)), with coherent material mass fraction covariances, are now allowed by the modelling, owing to equations (2.10) and (2.11). Moreover, the first-order model is an asymptotic approximation of the second-order model in the absence of an external sollicitation, and at long time scales, which is detailed in [35]. In this limit regime, the first-order and second-order models share common self-similar solutions (see Sects. 3.2.2 and 3.2.3). The closure modification amounts to introduce terms with a finite relaxation time which affects the transitory behaviour toward self-similar solutions. In situations where short time scales are at stake, this transient modelling is indeed crucial for reproducing physical phenomena under study. Second, the material mass fraction covariances, $\widehat{Y_{\alpha}^{\prime \prime} Y_{\beta}^{\prime \prime}}$, are also part of the evolved-intime correlations of the model. It is an important data as it can serve as a measure of the heterogeneity of the TMZ [33]. When turbulent combustion modelling comes into consideration [5,39], the model may thus provide with a modification of the combustion rates by the heterogeneity inside the TMZ [33]. Similarly, radiation transfer across a TMZ is modified by the heterogeneity level [11].

\section{Analysis of the COntinuous 1D MODEL}

In this Section, the continuous model is analysed, its important properties highlighted, having in view its discretization in the remainder of this article.

\subsection{The hyperbolic sub-system}

In this section, the closure with diffusion terms, having a superscript $c$, are dropped in equation (2.6) and system (2.10)-(2.13). 


\subsubsection{Realizability}

Proposition 3.1. Let $\mathcal{F}^{c}=0$ in equation (2.6). Consider $\mathbb{X}(t)$ a solution of this system, and assume that $\widetilde{\mathbf{U}}, \mathbb{X}, \mathbb{G}, \mathbb{H}$ are smooth enough so that all terms have classical meaning. Then, if $\mathbb{X}(0) \geq 0$, in the sense of symmetric matrices, $\mathbb{X}(t) \geq 0, \forall t>0$.

Proof. We first consider the case $\widetilde{\mathbf{U}}=0$. Then, defining

$$
\mathbb{K}(\mathbf{x}, t, s)=\exp \left(\int_{s}^{t} \mathrm{~d} r \mathbb{G}(\mathbf{x}, r)\right),
$$

one easily checks that

$$
\left\{\begin{array}{l}
\partial_{t} \mathbb{K}(\mathbf{x}, t, s)=\mathbb{G}(\mathbf{x}, t) \mathbb{K}(\mathbf{x}, t, s) \\
\mathbb{K}(\mathbf{x}, s, s)=\mathbb{I}
\end{array}\right.
$$

where $\mathbb{I}$ is the identity matrix. Therefore, the solution $\mathbb{X}$ of $(2.6)$ reads

$$
\mathbb{X}(\mathbf{x}, t)=\mathbb{K}(\mathbf{x}, t, 0) \mathbb{X}(\mathbf{x}, 0) \mathbb{K}^{t}(\mathbf{x}, t, 0)+\int_{0}^{t} \mathrm{~d} s \mathbb{K}(\mathbf{x}, t, s) \mathbb{H}(\mathbf{x}, s) \mathbb{H}^{t}(\mathbf{x}, s) \mathbb{K}^{t}(\mathbf{x}, t, s) .
$$

In the above sum, both terms are semi-definite positive, hence $\mathbb{X}(\mathbf{x}, t) \geq 0$.

Next, we turn to the case where $\widetilde{\mathbf{U}} \neq 0$. Equation (3.1) is then valid if one follows the characteristics of the field $\widetilde{\mathbf{U}}$. More precisely, let us define $\mathcal{C}=\mathcal{C}(\mathbf{x}, t)$ the solution to

$$
\left\{\begin{array}{l}
\partial_{t} \mathcal{C}(\mathbf{x}, t)=\widetilde{\mathbf{U}}(\mathcal{C}, t) \\
\mathcal{C}(\mathbf{x}, 0)=\mathbf{x}
\end{array}\right.
$$

and

$$
\mathbb{K}(\mathbf{x}, t, s)=\exp \left(\int_{s}^{t} \mathrm{~d} r \mathbb{G}(\mathcal{C}(\mathbf{x}, r), r)\right)
$$

Then we have

$$
\left\{\begin{array}{l}
\partial_{t} \mathbb{K}(\mathbf{x}, t, s)=\mathbb{G}(\mathcal{C}(\mathbf{x}, t), t) \mathbb{K}(\mathbf{x}, t, s) \\
\mathbb{K}(\mathbf{x}, s, s)=\mathbb{I}
\end{array}\right.
$$

and

$$
\mathbb{X}(\mathcal{C}(\mathbf{x}, t), t)=\mathbb{K}(\mathbf{x}, t, 0) \mathbb{X}(\mathbf{x}, 0) \mathbb{K}^{t}(\mathbf{x}, t, 0)+\int_{0}^{t} \mathrm{~d} s \mathbb{K}(\mathbf{x}, t, s) \mathbb{H}(\mathcal{C}(\mathbf{x}, s), s) \mathbb{H}^{t}(\mathcal{C}(\mathbf{x}, s), s) \mathbb{K}^{t}(\mathbf{x}, t, s)
$$

Therefore, we deduce that $\mathbb{X}(\mathcal{C}(\mathbf{x}, t), t) \geq 0$. In order to infer that $\mathbb{X}(\mathbf{x}, t) \geq 0$ for all $\mathbf{x}$, we only need to check that any $\mathbf{x}$ satisfies $\mathbf{x}=\mathcal{C}\left(\mathbf{x}_{0}, t\right)$, for some $\mathbf{x}_{0}$. In order to prove this, we consider the backward characteristics

$$
\left\{\begin{array}{l}
\partial_{s} \mathcal{D}(\mathbf{x}, s)=\widetilde{\mathbf{U}}(\mathcal{D}, s) \\
\mathcal{D}(\mathbf{x}, t)=\mathbf{x}
\end{array}\right.
$$

Since $\widetilde{\boldsymbol{U}}$ is smooth enough, the map $s \mapsto \mathcal{D}(\mathbf{x}, s)$ is defined for all $s$. Setting $\mathbf{x}_{\mathbf{0}}=\mathcal{D}(\mathbf{x},-t)$ ensures that $\mathbf{x}=\mathcal{C}\left(\mathbf{x}_{0}, t\right)$, which concludes the proof.

Remark 3.2. The smoothness assumption in Proposition 3.1 is important for two things: first, smoothness on $\mathbb{X}, \mathbb{G}, \mathbb{H}$ allows to write down the equation in a strong form and use Duhamel formula (3.1). Second, smoothness of $\widetilde{\boldsymbol{U}}$ allows to follow the characteristics backward, since it exists for all time. We do not know how to prove such a result in a non-smooth case. However, as we will see below (Prop. 3.6), the particular solution of the Riemann problem we use to build the scheme does satisfy the realizability property. 


\subsubsection{Hyperbolicity}

Let us introduce $\mathcal{I}_{\alpha \pm}=\widetilde{Y}_{\alpha} \pm \widetilde{Y_{\alpha}^{\prime \prime} u_{x}^{\prime \prime}} / \sqrt{\widetilde{u_{x}^{\prime \prime 2}}}, \lambda_{ \pm}= \pm \bar{\rho} x^{d} \sqrt{\widetilde{u_{x}^{\prime \prime 2}}}$, and $\lambda_{\alpha}=\bar{\rho} x^{d} \widetilde{Y_{\alpha}^{\prime \prime} u_{x}^{\prime \prime}} / \widetilde{Y}_{\alpha}$, where $d=0,1,2$ in planar, cylindrical or spherical coordinates, respectively.

Proposition 3.3. Consider System $(2.7)-(2.13)$ with $\mathcal{F}^{c}=0$ and $\left(\bar{\rho} \widetilde{\mathbf{u}^{\prime \prime}}\right)^{c}=0$ everywhere in space and time. This multi-species system is hyperbolic if and only if the mono-species, quasi-linear system (3.16), (3.17) is hyperbolic. Moreover, if $\widetilde{\mathbf{u}^{\prime \prime} \otimes \mathbf{u}^{\prime \prime}}>0$ and $\widetilde{Y}_{\alpha}>0$, System (3.16), (3.17) is hyperbolic, and all its characteristic fields are linearly degenerated $(L D)$. Its eigenvalues are $\widetilde{\mathbf{U}}, \widetilde{\mathbf{U}}+\lambda_{ \pm}$, and $\widetilde{\mathbf{U}}+\lambda_{\alpha}$, while the corresponding set of Riemann invariants, $\mathcal{W}_{o}, \mathcal{W}_{ \pm}$, and $\mathcal{W}_{\alpha}$, can be expressed as

$$
\begin{aligned}
& \mathcal{W}_{0}=\left\{\widetilde{Y}_{\alpha, i} / \widetilde{Y}_{\alpha}, \frac{1}{2}\left(\mathcal{I}_{\alpha+}+\mathcal{I}_{\alpha-}\right), \frac{\lambda_{+}}{2}\left(\mathcal{I}_{\alpha+}-\mathcal{I}_{\alpha-}\right)\right\}, \\
& \mathcal{W}_{ \pm}=\left\{\widetilde{Y}_{\alpha, i} / \widetilde{Y}_{\alpha}, \mathcal{I}_{\alpha \mp}, \lambda_{+}, \widetilde{Y_{\alpha}^{\prime \prime} u_{\perp}^{\prime \prime}}+\frac{1}{2 \lambda_{\mp}}\left(\mathcal{I}_{\alpha+}+\mathcal{I}_{\alpha-}\right) \bar{\rho} x^{d} \widetilde{u_{x}^{\prime \prime} u_{\perp}^{\prime \prime}}\right\}, \\
& \mathcal{W}_{\alpha}=\left\{\mathcal{I}_{\alpha+}, \mathcal{I}_{\alpha-}, \widetilde{Y_{\alpha}^{\prime \prime} u_{\perp}^{\prime \prime}}, \lambda_{+}\right\} .
\end{aligned}
$$

Proof. We find it useful to write the 1D system in mass coordinates, using the variable change $\mathrm{d} m=\bar{\rho} x^{d} \mathrm{~d} x$, where $x$ is one cartesian coordinate, or the radial coordinate. Then the system writes

$$
\begin{aligned}
& \frac{\mathrm{d}}{\mathrm{d} t} \widetilde{Y}_{\alpha}+\partial_{m}\left(\bar{\rho} x^{d} \widetilde{Y_{\alpha}^{\prime \prime} u_{x}^{\prime \prime}}\right)=0 \\
& \frac{\mathrm{d}}{\mathrm{d} t} \widetilde{Y}_{\alpha, i}+\partial_{m}\left(\bar{\rho} x^{d} \widetilde{Y_{\alpha}^{\prime \prime} u_{x}^{\prime \prime}} \frac{\widetilde{Y}_{\alpha, i}}{\widetilde{Y}_{\alpha}}\right)=0 \\
& \frac{\mathrm{d}}{\mathrm{d} t} \widetilde{Y_{\alpha}^{\prime \prime} \mathbf{u}^{\prime \prime}}+\bar{\rho} x^{d} \widetilde{\mathbf{u}^{\prime \prime} u_{x}^{\prime \prime}} \partial_{m} \widetilde{Y}_{\alpha}=-\frac{1}{2}\left(C_{\tau}+C_{1}\right) \widetilde{\omega} \widetilde{Y_{\alpha}^{\prime \prime} \mathbf{u}^{\prime \prime}}, \\
& \frac{\mathrm{d}}{\mathrm{d} t} \widetilde{Y_{\alpha}^{\prime \prime} Y_{\beta}^{\prime \prime}}+\bar{\rho} x^{d} \widetilde{Y_{\alpha}^{\prime \prime} u_{x}^{\prime \prime}} \partial_{m} \widetilde{Y}_{\beta}+\bar{\rho} x^{d} \widetilde{Y_{\alpha}^{\prime \prime} u_{x}^{\prime \prime}} \partial_{m} \widetilde{Y}_{\alpha}=-\left(C_{\tau}-C_{\tau o}\right) \widetilde{\omega} \widetilde{Y_{\alpha}^{\prime \prime} Y_{\beta}^{\prime \prime}} \text {, } \\
& \frac{\mathrm{d}}{\mathrm{d} t} \widetilde{\mathbf{u}^{\prime \prime} \otimes \mathbf{u}^{\prime \prime}}=-\frac{2}{3} \widetilde{\omega} \tilde{k} \mathbb{I}-C_{1} \widetilde{\omega}\left[\widetilde{\mathbf{u}^{\prime \prime} \otimes \mathbf{u}^{\prime \prime}}-\frac{2}{3} \widetilde{k} \mathbb{I}\right], \\
& \frac{\mathrm{d}}{\mathrm{d} t} \widetilde{\varepsilon}=-C_{\varepsilon 2} \widetilde{\omega} \widetilde{\varepsilon} .
\end{aligned}
$$

We rewrite the system (3.3)-(3.8) under the following compact, quasi-linear formulation

$$
\begin{aligned}
& \frac{\mathrm{d}}{\mathrm{d} t} \mathcal{I}_{\alpha \pm}+\lambda_{ \pm} \partial_{m} \mathcal{I}_{\alpha \pm} \pm \frac{1}{2}\left(\mathcal{I}_{\alpha+}-\mathcal{I}_{\alpha-}\right) \partial_{m} \lambda_{ \pm}=\mp \frac{1}{2}\left(\frac{1}{2} C_{\tau}+\frac{1}{3}\left(C_{1}-1\right) \widetilde{k} / \widetilde{u_{x}^{\prime 2}}\right) \widetilde{\omega}\left(\mathcal{I}_{\alpha+}-\mathcal{I}_{\alpha-}\right), \\
& \frac{\mathrm{d}}{\mathrm{d} t} \widetilde{Y_{\alpha}^{\prime \prime} u_{\perp}^{\prime \prime}}+\frac{1}{2} \bar{\rho} x^{d} \widetilde{u_{x}^{\prime \prime} u_{\perp}^{\prime \prime}} \partial_{m}\left(\mathcal{I}_{\alpha+}+\mathcal{I}_{\alpha-}\right)=-\frac{1}{2}\left(C_{\tau}+C_{1}\right) \widetilde{\omega} \widetilde{Y_{\alpha}^{\prime \prime} u_{\perp}^{\prime \prime}},
\end{aligned}
$$

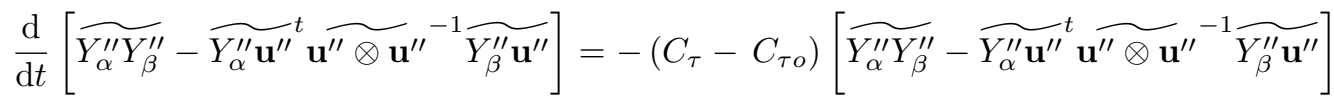

$$
\begin{aligned}
& +C_{\tau o}{\widetilde{Y_{\alpha}^{\prime \prime} \mathbf{u}^{\prime \prime}}}^{t}{\widetilde{\mathbf{u}^{\prime \prime} \otimes \mathbf{u}^{\prime \prime}}}^{-1} \widetilde{Y_{\beta}^{\prime \prime} \mathbf{u}^{\prime \prime}} \\
& +\frac{2}{3}\left(C_{1}-1\right) \widetilde{\omega} \widetilde{k}{\widetilde{Y_{\alpha}^{\prime \prime} \mathbf{u}^{\prime \prime}}}^{t}{\widetilde{\mathbf{u}^{\prime \prime} \otimes \mathbf{u}^{\prime \prime}}}^{-2} \widetilde{Y_{\beta}^{\prime \prime} \mathbf{u}^{\prime \prime}} \\
& \frac{\mathrm{d}}{\mathrm{d} t}\left(\frac{\widetilde{Y}_{\alpha, i}}{\widetilde{Y}_{\alpha}}\right)+\lambda_{\alpha} \partial_{m}\left(\frac{\widetilde{Y}_{\alpha, i}}{\widetilde{Y}_{\alpha}}\right)=0,
\end{aligned}
$$




$$
\begin{aligned}
\frac{\mathrm{d}}{\mathrm{d} t} \widetilde{\mathbf{u}^{\prime \prime} \otimes \mathbf{u}^{\prime \prime}} & =-\frac{2}{3} \widetilde{\omega} \widetilde{k} \mathbb{I}-C_{1} \widetilde{\omega}\left[\widetilde{\mathbf{u}^{\prime \prime} \otimes \mathbf{u}^{\prime \prime}}-\frac{2}{3} \widetilde{k} \mathbb{I}\right], \\
\frac{\mathrm{d}}{\mathrm{d} t} \widetilde{\varepsilon} & =-C_{\varepsilon 2} \widetilde{\omega} \widetilde{\varepsilon},
\end{aligned}
$$

from which we deduce straightforwardly the eigenvalues of the system (3.3)-(3.8): $\widetilde{\mathbf{U}}, \widetilde{\mathbf{U}}+\lambda_{ \pm}$, and $\widetilde{\mathbf{U}}+\lambda_{\alpha}$. Here, the subscript $\perp \in\{y, z\}$ refers to any transverse component of the mass fraction flux.

Since the model is realizable, all the eigenvalues are real-valued. The model reformulation (3.9)-(3.14) makes it also clear that hyperbolicity can be studied for each material independently, discarding equation (3.11). The latter can indeed be viewed as a diagnostic (one-way coupled) equation, whose eigenvalue is $\widetilde{\mathbf{U}}$. We can also isolate the components of the Reynolds tensor, in equation (3.13), that are independent from the other variables of the quasi-linear system (the eigenvalue associated to these components is also $\widetilde{\mathbf{U}}$ ). The equation for the remaining component, $\lambda_{+}=-\lambda_{-}$, can be written

$$
\frac{\mathrm{d}}{\mathrm{d} t} \lambda_{+}=0
$$

The dissipation rate of the turbulent kinetic energy, $\widetilde{\varepsilon}$, obtained from equation (3.14) of the quasi-linear system, is independent from the other variables of this quasi-linear system as well. The associated eigenvalue is also $\widetilde{\mathbf{U}}$. Therefore, hyperbolicity of the model (3.3)-(3.8) is equivalent to the hyperbolicity of the reduced, mono-species system of quasi-linear equations (3.9)-(3.10)-(3.12)-(3.15). The latter can be rewritten as

$$
\frac{\mathrm{d}}{\mathrm{d} t} \mathcal{Q}_{\alpha}+A \partial_{m} \mathcal{Q}_{\alpha}=0
$$

with $\mathcal{Q}_{\alpha}=\left(\mathcal{I}_{\alpha+}, \mathcal{I}_{\alpha-}, \widetilde{Y_{\alpha}^{\prime \prime} u_{\perp}^{\prime \prime}}, \lambda_{+}, \widetilde{Y}_{\alpha, i} / \widetilde{Y}_{\alpha}\right)^{t}$,

$$
A=\left(\begin{array}{ccccc}
\lambda_{+} & 0 & 0 & \frac{\mathcal{I}_{\alpha+}-\mathcal{I}_{\alpha-}}{2} & \mathbf{0}^{t} \\
0 & -\lambda_{+} & 0 & \frac{\mathcal{I}_{\alpha+}-\mathcal{I}_{\alpha-}}{2} & \mathbf{0}^{t} \\
\frac{1}{2} \bar{\rho} x^{d} \widetilde{u_{x}^{\prime \prime} u_{\perp}^{\prime \prime}} & \frac{1}{2} \bar{\rho} x^{d} \widetilde{u_{x}^{\prime \prime} u_{\perp}^{\prime \prime}} & 0 & 0 & \mathbf{0}^{t} \\
0 & 0 & 0 & 0 & \mathbf{0}^{t} \\
\mathbf{0} & \mathbf{0} & \mathbf{0} & \mathbf{0} & \lambda_{\alpha} \mathbf{I}_{\alpha}
\end{array}\right),
$$

where $\mathbf{I}_{\alpha}$ is the identity matrix whose size is equal to the number of constituents in the material $\alpha$.

The eigenvalues of the matrix $A$ are $\widetilde{\mathbf{U}}, \widetilde{\mathbf{U}}+\lambda_{ \pm}$, and $\widetilde{\mathbf{U}}+\lambda_{\alpha}$, with respective multiplicity equal to 2,1 , and to the number of material constituents in the material $\alpha$. Their right eigenvectors are

$$
\left\{\left(-\frac{\mathcal{I}_{\alpha+}-\mathcal{I}_{\alpha-}}{2 \lambda_{+}}, \frac{\mathcal{I}_{\alpha+}-\mathcal{I}_{\alpha-}}{2 \lambda_{+}}, 0,1, \mathbf{0}\right)^{t},(0,0,1,0, \mathbf{0})^{t}\right\},
$$

for the eigenvalue $\widetilde{\mathbf{U}}$,

$$
\left\{\left(\lambda_{+}, 0, \frac{1}{2} \bar{\rho} x^{d} \widetilde{u_{x}^{\prime \prime} u_{\perp}^{\prime \prime}}, 0, \mathbf{0}\right)^{t}\right\} \text { and }\left\{\left(0,-\lambda_{+}, \frac{1}{2} \bar{\rho} x^{d} \widetilde{u_{x}^{\prime \prime} u_{\perp}^{\prime \prime}}, 0, \mathbf{0}\right)^{t}\right\},
$$

respectively, for the eigenvalues $\widetilde{\mathbf{U}}+\lambda_{+}$and $\widetilde{\mathbf{U}}+\lambda_{-}$,

$$
\left\{\left(0,0,0,0, \mathbf{e}_{i}\right)^{t}, \forall i\right\},
$$


for the eigenvalue $\widetilde{\mathbf{U}}+\lambda_{\alpha}$, with $\mathbf{e}_{i}=\left(\delta_{1 i}, \delta_{2 i}, \cdots\right)^{t}$, using the Kronecker symbol $\delta$.

The determinant of the matrix composed by these right eigenvectors (the change-of-frame matrix to the eigenspace) can be computed, and is equal to $\lambda_{+} \lambda_{-}$. Therefore, this determinant is positive provided $\widetilde{u_{x}^{\prime \prime 2}}$ is non-null, that is inside the TMZ. This completes the proof of hyperbolicity.

Finally, the field associated to the eigenvalue $\widetilde{\mathbf{U}}+\lambda_{\alpha}$ is LD because it is a function of all $\mathcal{Q}_{\alpha}$ field but $\widetilde{Y}_{\alpha, i} / \widetilde{Y}_{\alpha}$, and due to the structure (non-null components) of the corresponding eigenvector. Moreover, the field associated to the eigenvalue $\widetilde{\mathbf{U}}+\lambda_{ \pm}$is LD since $\nabla_{\mathcal{Q}_{\alpha}}\left(\widetilde{\mathbf{U}}+\lambda_{ \pm}\right)=(0,0,0,1, \mathbf{0})^{t}$ is orthogonal to the eigenvector of $\widetilde{\mathbf{U}}+\lambda_{ \pm}$. Therefore all the fields of the system are LD.

\subsubsection{Entropies}

Proposition 3.4. Smooth enough solutions of system (3.3)-(3.8) satisfy the following couple of mathematical entropy relations

$$
\left\{\frac{\mathrm{d}}{\mathrm{d} t} \eta_{\alpha}^{a}+\partial_{m} \psi_{\alpha}=\mathcal{D}_{\alpha}^{a} \leq 0, \frac{\mathrm{d}}{\mathrm{d} t} \eta_{\alpha}^{b}+\partial_{m} \psi_{\alpha}=\mathcal{D}_{\alpha}^{b} \leq 0\right\}
$$

for each material, denoted by the subscript $\alpha$. The entropy expressions read

$$
\left\{\eta_{\alpha}^{a}=\eta^{a}\left(\widetilde{Y}_{\alpha}, \widetilde{Y_{\alpha}^{\prime \prime 2}}\right)=\frac{1}{2}\left(\widetilde{Y_{\alpha}^{\prime \prime 2}}+\widetilde{Y}_{\alpha}^{2}\right), \eta_{\alpha}^{b}=\eta^{b}\left(\widetilde{Y}_{\alpha}, \widetilde{Y_{\alpha}^{\prime \prime} \mathbf{u}^{\prime \prime}}, \widetilde{\mathbf{u}^{\prime \prime} \otimes \mathbf{u}^{\prime \prime}}\right)=\frac{1}{2}\left(\widetilde{Y}_{\alpha}^{2}+\widetilde{Y_{\alpha}^{\prime \prime} \mathbf{u}^{\prime \prime}} \stackrel{\mathbf{u}^{\prime \prime} \otimes \mathbf{u}^{\prime \prime}}{-1} \widetilde{Y_{\alpha}^{\prime \prime} \mathbf{u}^{\prime \prime}}\right)\right\}
$$

Both share the same entropy flux, $\psi_{\alpha}=\psi\left(\widetilde{Y}_{\alpha}, \widetilde{Y_{\alpha}^{\prime \prime} \mathbf{u}^{\prime \prime}}\right)=\bar{\rho} x^{d} \widetilde{Y}_{\alpha} \widetilde{Y_{\alpha}^{\prime \prime} \mathbf{u}^{\prime \prime}}$, while they have distinct negative entropy dissipation rates

$$
\left\{\mathcal{D}_{\alpha}^{a}=-\left(C_{\tau}-C_{\tau o}\right) \widetilde{\omega} \widetilde{Y_{\alpha}^{\prime \prime 2}}, \mathcal{D}_{\alpha}^{b}=-\frac{1}{3}\left(C_{1}-1\right) \widetilde{\omega} \widetilde{k Y_{\alpha}^{\prime \prime} \mathbf{u}^{\prime \prime}} t^{t}\left(\mathbf{u}^{\prime \prime} \otimes \mathbf{u}^{\prime \prime}\right)^{-2} \widetilde{Y_{\alpha}^{\prime \prime} \mathbf{u}^{\prime \prime}}-\frac{1}{2} C_{\tau} \widetilde{\omega} \widetilde{Y_{\alpha}^{\prime \prime} \mathbf{u}^{\prime \prime}}\left(\mathbf{u}^{\prime \prime} \otimes \mathbf{u}^{\prime \prime}\right)^{-1} \widetilde{Y_{\alpha}^{\prime \prime} \mathbf{u}^{\prime \prime}}\right\} .
$$

Proof. The Reynolds tensor, $\widetilde{\mathbf{u}^{\prime \prime} \otimes \mathbf{u}^{\prime \prime}}$, is nonsingular inside the TMZ. Moreover, its derivatives can be obtained with the relation

$$
\partial{\widetilde{\mathbf{u}^{\prime \prime} \otimes \mathbf{u}^{\prime \prime}}}^{-1}=-{\widetilde{\mathbf{u}^{\prime \prime} \otimes \mathbf{u}^{\prime \prime}}}^{-1} \partial{\widetilde{\mathbf{u}^{\prime \prime} \otimes \mathbf{u}^{\prime \prime} \mathbf{u}^{\prime \prime} \otimes \mathbf{u}^{\prime \prime}}}^{-1}
$$

provided it is smooth enough. Hence $\widetilde{\mathbf{u}^{\prime \prime} \otimes \mathbf{u}^{\prime \prime}}{ }^{-1}$ and its derivatives are well-defined quantities.

The next step is to prove that both functions $\eta_{\alpha}^{a}$ and $\eta_{\alpha}^{b}$ are convex functions.

Concerning $\eta_{\alpha}^{a}$, it is indeed convex, because it is the sum of two convex functions. As such, and also because $\mathcal{D}_{\alpha}^{a}$ is non-positive, it is an entropy for the system (3.3)-(3.8).

Next, the convexity of $\eta_{\alpha}^{b}$ can be deduced from the convexity of the following function:

$$
\begin{aligned}
\mathcal{N}: \mathbb{R}^{d} \times D P(d) & \rightarrow \mathbb{R} \\
(\mathbf{x}, \mathbf{M}) & \mapsto \mathbf{x}^{t} \mathbf{M}^{-1} \mathbf{x}
\end{aligned}
$$

where $D P(d)$ is the positive definite cone for matrices of size $d$. The proof starts by noticing that the following function

$$
\begin{aligned}
\eta: \mathbb{R} \times \mathbb{R}_{+}^{*} & \rightarrow \mathbb{R} \\
(x, m) & \mapsto x^{2} / m
\end{aligned}
$$

is convex because its Hessian matrix has only non-negative eigenvalues. Then, the spectral theorem can be used to show that, given $M, N \in D P(d)$, there exists a matrix $P$ which satisfies $M=P P^{t}$ and $N=P D P^{t}$, where $D=\operatorname{diag}\left(d_{1}, \cdots, d_{d}\right)$ is a diagonal matrix. Let $\mathbf{x}, \mathbf{y} \in \mathbb{R}^{d}$. We obtain

$$
\mathcal{N}(\mathbf{x}, \mathbf{M})=\|\hat{\mathbf{x}}\|^{2}=\sum_{i=1}^{d} \eta\left(\hat{x}_{i}, 1\right) \quad ; \quad \mathcal{N}(\mathbf{y}, \mathbf{N})=\left\|D^{-1} \hat{\mathbf{y}}\right\|^{2}=\sum_{i=1}^{d} \eta\left(\hat{y}_{i}, d_{i}\right)
$$


where $\hat{\mathbf{x}}=P^{-1} \mathbf{x}=\left(\hat{x}_{1}, \cdots, \hat{x}_{d}\right)$ and $\hat{\mathbf{y}}=P^{-1} \mathbf{y}=\left(\hat{y}_{1}, \cdots, \hat{y}_{d}\right)$. Introducing $\nu \in(0,1)$, we finally get

$$
\begin{aligned}
\mathcal{N}(\nu \mathbf{x}+(1-\nu) \mathbf{y}, \nu \mathbf{M}+(1-\nu) \mathbf{N}) & =(\nu \hat{\mathbf{x}}+(1-\nu) \hat{\mathbf{y}})^{t}\left[\nu \mathbb{I}_{d}+(1-\nu) D\right]^{-1}(\nu \hat{\mathbf{x}}+(1-\nu) \hat{\mathbf{y}}), \\
& =\sum_{i=1}^{d} \frac{\left[\nu \hat{x}_{i}+(1-\nu) \hat{y}_{i}\right]^{2}}{\nu+(1-\nu) d_{i}} \\
& =\sum_{i=1}^{d} \eta\left[\nu \hat{x}_{i}+(1-\nu) \hat{y}_{i}, \nu+(1-\nu) d_{i}\right] \\
& \leq \nu \mathcal{N}(\mathbf{x}, \mathbf{M})+(1-\nu) \mathcal{N}(\mathbf{y}, \mathbf{N})
\end{aligned}
$$

which has been obtained from the convexity of the function $\eta$ applied on each index $i$. Hence, the function $\mathcal{N}$ is convex, and so is $\eta_{\alpha}^{b}$. Since $\mathcal{D}_{\alpha}^{b} \leq 0,\left(\eta_{\alpha}^{b}, \psi_{\alpha}\right)$ is also a couple of entropy and entropy flux.

\subsubsection{The stationary states}

Proposition 3.5. If $C_{\varepsilon 2} \neq 1$, the stationary states of System (3.3)-(3.8) satisfy

$$
\begin{aligned}
\bar{\rho} x^{d} \widetilde{Y_{\alpha}^{\prime \prime} u_{x}^{\prime \prime}} & =\left[\bar{\rho} x^{d} \widetilde{Y_{\alpha}^{\prime \prime} u_{x}^{\prime \prime}}\right]^{o}, \\
\widetilde{Y}_{\alpha} & =\widetilde{Y}_{\alpha}^{o}, \\
\widetilde{Y}_{\alpha, i} & =\widetilde{Y}_{\alpha, i}^{o},
\end{aligned}
$$

where $\widetilde{Y}_{\alpha}^{o}, \widetilde{Y}_{\alpha, i}^{o}$ and $\left[\bar{\rho} x^{d} \widetilde{Y_{\alpha}^{\prime \prime} u_{x}^{\prime \prime}}\right]^{o}$ are constants with respect to the time and mass coordinates.

Proof. Combining equations (3.7) and (3.8), we obtain $\frac{\mathrm{d}}{\mathrm{d} t} \widetilde{\omega}=-\left(C_{\varepsilon 2}-1\right) \widetilde{\omega}^{2}$. Hence, stationary states of system system (3.3)-(3.8) must satisfy $\widetilde{\omega}=0$ if $C_{\varepsilon 2} \neq 1$.

\subsubsection{D Riemann problem in curvilinear coordinates: resolution and decomposition}

Proposition 3.6. Assume $d=0,1,2$ and consider System (2.7)-(2.8)-(2.10)-(2.11)-(2.12), where the righthand side and diffusion terms are set to zero. The system reduces to System (2.7)-(3.3)-(3.5)-(3.6)-(3.7), with null right-hand side, in mass coordinates. This system admits a four-state piecewise constant solution of a Riemann problem in the mass coordinates, that is:

$$
\left(\bar{\rho} x^{d}, \widetilde{Y}_{\alpha}, \bar{\rho} x^{d} \mathbb{X}\right)= \begin{cases}\left(\left[\bar{\rho} x^{d}\right]^{L},\left.\widetilde{Y}_{\alpha}\right|^{L},[\bar{\rho} x d \mathbb{X}]^{L}\right) & \text { if } m-m_{0}<-\left[\bar{\rho} x^{d} \sqrt{\widetilde{u_{x}^{\prime 2}}}\right]^{L} t, \\ \left(\left[\bar{\rho} x^{d}\right]^{L^{*}},\left.\tilde{Y}_{\alpha}\right|^{L^{*}},\left[\bar{\rho} x^{d} \mathbb{X}\right]^{L^{*}}\right) & \text { if }-\left[\bar{\rho} x^{d} \sqrt{\overline{u_{x}^{\prime \prime 2}}}\right]^{L} t<m-m_{0}<0, \\ \left(\left[\bar{\rho} x^{d}\right]^{R^{*}},\left.\widetilde{Y}_{\alpha}\right|^{R^{*}},\left[\bar{\rho} x^{d} \mathbb{X}\right]^{R^{*}}\right) & \text { if } 0<m-m_{0}<\left[\bar{\rho} x^{d} \sqrt{\widetilde{u_{x}^{\prime \prime 2}}}\right]^{R} t, \\ \left(\left[\bar{\rho} x^{d}\right]^{R},\left.\widetilde{Y}_{\alpha}\right|^{R},\left[\bar{\rho} x^{d} \mathbb{X}\right]^{R}\right) & \text { if } m-m_{0}>\left[\bar{\rho} x^{d} \sqrt{\overline{u_{x}^{\prime \prime 2}}}\right]^{R} t,\end{cases}
$$

where the intermediated states, $\left(\left.\bar{\rho}\right|^{L^{*}},\left.\widetilde{Y}_{\alpha}\right|^{L^{*}},\left[\bar{\rho} x^{d} \mathbb{X}\right]^{L^{*}}\right)$ and $\left(\left.\bar{\rho}\right|^{R^{*}},\left.\widetilde{Y}_{\alpha}\right|^{R^{*}},\left[\bar{\rho} x^{d} \mathbb{X}\right]^{R^{*}}\right)$ are defined as the unique solution of the algebraic system

$$
\begin{aligned}
& {\left[\bar{\rho} x^{d}\right]^{L^{*}}=\left[\bar{\rho} x^{d}\right]^{L},} \\
& {\left[\bar{\rho} x^{d}\right]^{R^{*}}=\left[\bar{\rho} x^{d}\right]^{R},}
\end{aligned}
$$




$$
\begin{aligned}
& {\left[\bar{\rho} x^{d} \sqrt{\widetilde{u_{x}^{\prime \prime 2}}}\right]^{*} R=\left[\bar{\rho} x^{d} \sqrt{\widetilde{u_{x}^{\prime \prime 2}}}\right]^{R}} \\
& {\left[\bar{\rho} x^{d} \sqrt{\widetilde{u_{x}^{\prime \prime 2}}}\right]^{*}=\left[\bar{\rho} x^{d} \sqrt{\widetilde{u_{x}^{\prime \prime 2}}}\right]^{L} \text {. }} \\
& \left.\tilde{Y}_{\alpha}\right|^{* L}=\left.\tilde{Y}_{\alpha}\right|^{*} R=\left.\tilde{Y}_{\alpha}\right|^{*}, \\
& {\left[\bar{\rho} x^{d} \widetilde{Y_{\alpha}^{\prime \prime} u_{x}^{\prime \prime}}\right]^{*} L=\left[\bar{\rho} x^{d} \widetilde{Y_{\alpha}^{\prime \prime} u_{x}^{\prime \prime}}\right]^{*}=\left[\bar{\rho} x^{d} \widetilde{Y_{\alpha}^{\prime \prime} u_{x}^{\prime \prime}}\right]^{*} \text {. }} \\
& {\left[\left.\bar{\rho} x^{d}{\sqrt{\widetilde{u_{x}^{\prime \prime 2}}}}^{*} R \widetilde{Y}_{\alpha}\right|^{*} R-\left[\bar{\rho} x^{d} \widetilde{Y_{\alpha}^{\prime \prime} u_{x}^{\prime \prime}}\right]^{*} R=\left.\left[\bar{\rho} x^{d} \sqrt{\widetilde{u_{x}^{\prime \prime 2}}}\right]^{R} \widetilde{Y}_{\alpha}\right|^{R}-\left[\bar{\rho} x^{d} \widetilde{Y_{\alpha}^{\prime \prime} u_{x}^{\prime \prime}}\right]^{R},\right.} \\
& {\left.\left[\bar{\rho} x^{d} \sqrt{\widetilde{u_{x}^{\prime \prime 2}}}\right]^{*} \tilde{Y}_{\alpha}\right|^{*}+\left[\bar{\rho} x^{d} \widetilde{Y_{\alpha}^{\prime \prime} u_{x}^{\prime \prime}}\right]^{*}=\left.\left[\bar{\rho} x^{d} \sqrt{\widetilde{u_{x}^{\prime \prime 2}}}\right]^{L} \widetilde{Y}_{\alpha}\right|^{L}+\left[\bar{\rho} x^{d} \widetilde{Y_{\alpha}^{\prime \prime} u_{x}^{\prime \prime}}\right]^{L},} \\
& {\left[\bar{\rho} x^{d} \widetilde{Y_{\alpha}^{\prime \prime} Y_{\beta}^{\prime \prime}}\right]^{*}-\left(\left[\bar{\rho} x^{d} \widetilde{Y_{\alpha}^{\prime \prime} u_{x}^{\prime \prime}}\right]^{t}\right)^{2} /\left[\bar{\rho} x^{d} \widetilde{u_{x}^{\prime \prime 2}}\right]^{*}=\left[\bar{\rho} x^{d} \widetilde{Y_{\alpha}^{\prime \prime} Y_{\beta}^{\prime \prime}}\right]^{L}-\left(\left[\bar{\rho} x^{d} \widetilde{Y_{\alpha}^{\prime \prime} u_{x}^{\prime \prime}}\right]^{L}\right)^{2} /\left[\bar{\rho} x^{d} \widetilde{u_{x}^{\prime \prime}}\right]^{L},} \\
& {\left[\bar{\rho} x^{d} \widetilde{Y_{\alpha}^{\prime \prime} Y_{\beta}^{\prime \prime}}\right]^{*}-\left(\left[\bar{\rho} x^{d} \widetilde{Y_{\alpha}^{\prime \prime} u_{x}^{\prime \prime}}\right]^{*}\right)^{2} /\left[\bar{\rho} x^{d} \widetilde{u_{x}^{\prime \prime}}\right]^{*}=\left[\bar{\rho} x^{d} \widetilde{Y_{\alpha}^{\prime \prime} Y_{\beta}^{\prime \prime}}\right]^{R}-\left(\left[\bar{\rho} x^{d} \widetilde{Y_{\alpha}^{\prime \prime} u_{x}^{\prime \prime}}\right]^{R}\right)^{2} /\left[\bar{\rho} x^{d} \widetilde{u_{x}^{\prime \prime}}\right]^{R}}
\end{aligned}
$$

with $\left[\bar{\rho} x^{d} \widetilde{u_{x}^{\prime \prime 2}}\right]^{S}=\left(\left[\bar{\rho} x^{d} \sqrt{\widetilde{u_{x}^{\prime \prime}}}\right]^{S}\right)^{2} /\left[\bar{\rho} x^{d}\right]^{S}$, and $S$ can be either $L, R, L^{*}$ or $R^{*}$.

This solution is realizable, in the sense that if $\left[\bar{\rho} x^{d} \mathbb{X}\right]^{L},\left[\bar{\rho} x^{d} \mathbb{X}\right]^{R} \geq 0$, then $\left[\bar{\rho} x^{d} \mathbb{X}\right]^{*},\left[\bar{\rho} x^{d} \mathbb{X}\right]^{*} R \geq 0$. It is also isentropic, in the sense that it is solution of system (3.20), with $\mathcal{D}_{\alpha}^{a}=\mathcal{D}_{\alpha}^{b}=0$.

Proof. In order to adress simultaneously configurations with $d=0,1,2$, System $(2.7)-(2.8)-(2.10)-(2.11)-(2.12)$ is considered in mass coordinates, without loss of generality. We are left with System (2.7)-(3.3)-(3.5)-(3.6)(3.7), where we set the right-hand side to zero. If the initial state is realizable, then the algebraic System (3.22)-(3.31) admits a unique solution. The positivity of $\left[\bar{\rho} x^{d} \mathbb{X}\right]^{*} L$ and $\left[\bar{\rho} x^{d} \mathbb{X}\right]^{*} R$ can be obtained using the decomposition (4.24) of correlation tensors. Equation (3.3) is satisfied in a weak sense by this particular solution, that is

$$
\int_{0}^{T} \mathrm{~d} t \int_{0}^{\infty} \mathrm{d} m\left[\partial_{t} \widetilde{Y}_{\alpha}+\partial_{m}\left[\bar{\rho} x^{d} \widetilde{Y_{\alpha}^{\prime \prime} u_{x}^{\prime \prime}}\right]\right] \varphi(t, m)=0,
$$

for any test function $\varphi$ smooth enough. Here, $T$ is a time such as $m_{0}-\left[\bar{\rho} x^{d \sqrt{u^{\prime \prime 2}}}\right]^{L} T>0$, where $m_{0}$ is the position that initially makes the separation between the $L$ and $R$ states. This can be shown classically by first integrating equation (3.32) by parts, then inserting the solution of System (3.22)-(3.31) and integrating the derivatives of the test function. Such procedure cannot be applied a priori for equation (3.5), because it has a non-conservative product. However, this difficulty can be circumvented, noticing that equation (3.5) with null right-hand side is satisfied in a strong sense, at $m=m_{0}$, and for all times, by the solution of System (3.22)(3.31). Therefore, it is sufficient to show that equation (3.5) is satisfied in a weak sense on $\left[0, m_{0}[\right.$ and $] m_{0}, \infty[$ separately, which writes

$$
\begin{gathered}
\int_{0}^{T} \mathrm{~d} t \int_{0}^{m_{0}} \mathrm{~d} m\left[\partial_{t} \widetilde{Y_{\alpha}^{\prime \prime} u_{x}^{\prime \prime}}+\left[\bar{\rho} x^{d} \widetilde{u_{x}^{\prime \prime 2}}\right] \partial_{m} \widetilde{Y}_{\alpha}\right] \varphi(t, m)=0, \\
\int_{0}^{T} \mathrm{~d} t \int_{m_{0}}^{\infty} \mathrm{d} m\left[\partial_{t} \widetilde{Y_{\alpha}^{\prime \prime} u_{x}^{\prime \prime}}+\left[\bar{\rho} x^{d} \widetilde{u_{x}^{\prime \prime 2}}\right] \partial_{m} \widetilde{Y}_{\alpha}\right] \varphi(t, m)=0,
\end{gathered}
$$




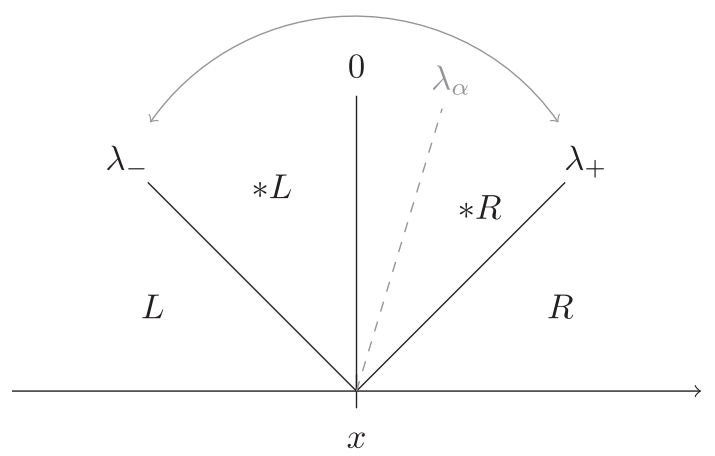

FiguRE 1. Riemann fan associated to the system (3.9)-(3.14), in a 1D geometry, with transverse symmetry in cartesian coordinates, assuming $\widetilde{\mathbf{U}}=0$.

for any test function $\varphi$ smooth enough. This is true because the integration by parts is allowed, even with the presence of a non-conservative product, because $\left[\bar{\rho} x^{d} \widehat{u_{x}^{\prime \prime 2}}\right]$ is smooth (constant) on both domains, owing to the solution of System (3.22)-(3.31). Next, equations (3.7) and (3.11), with null right-hand sides, are satisfied in a strong sense by the solution of System (3.22)-(3.31). Moreover, equation (3.6) with null right-hand side can be written as a combination of equations (3.5), (3.7) and (3.11) (with null right-hand sides), which are satisfied in a weak sense by the solution of System (3.22)-(3.31). Therefore, the solution of System (3.22)-(3.31) satisfies equation (3.6), with null right-hand side, in a weak sense.

As a prelude to the discretization step, several remarks can be done.

Remark 3.7. The particular solution of the Riemann problem that is exhibited in Proposition 3.6 can be built using the Linearly Degenerated (LD) stucture of the system of interest. The continuity of Riemann invariants is expressed across the waves having speed $\widetilde{\mathbf{U}}+\lambda_{ \pm}$and $\widetilde{\mathbf{U}}$, as illustrated in Figure 1. Even in this LD case, we cannot rely, to our knowledge, on a well-established theoretical result, that would grant a unique solution of the Riemann problem (for such a non-strictly hyperbolic, non-conservative system). However, it has been shown a posteriori that the solution of (3.22)-(3.31) is indeed a solution of the Riemann problem. Conversely, every four-state piecewise constant solution of the Riemann problem must satisfy the Riemann invariant continuity relations (3.22)-(3.31).

Remark 3.8. The algebraic system (3.22)-(3.31) can be solved in two steps. The first steps amounts to solve System (3.24)-(3.29), that can be put in terms of a two-equation system

$$
\begin{gathered}
{\left[\left.\left.\bar{\rho} x^{d} \sqrt{\widetilde{u_{x}^{\prime 2}}}\right|^{R} \tilde{Y}_{\alpha}\right|^{*}-\left[\bar{\rho} x^{d} \widetilde{Y_{\alpha}^{\prime \prime} u_{x}^{\prime \prime}}\right]^{*}=\left[\left.\left.\bar{\rho} x^{d} \sqrt{\widetilde{u_{x}^{\prime \prime 2}}}\right|^{R} \widetilde{Y}_{\alpha}\right|^{R}-\left[\bar{\rho} x^{d} \widetilde{Y_{\alpha}^{\prime \prime} u_{x}^{\prime \prime}}\right]^{R},\right.\right.} \\
{\left.\left[\bar{\rho} x^{d} \sqrt{\widetilde{u_{x}^{\prime \prime 2}}}\right]^{L} \tilde{Y}_{\alpha}\right|^{*}+\left[\bar{\rho} x^{d} \widetilde{Y_{\alpha}^{\prime \prime} u_{x}^{\prime \prime}}\right]^{*}=\left[\left.\left.\bar{\rho} x^{d} \sqrt{\widetilde{u_{x}^{\prime \prime 2}}}\right|^{L} \widetilde{Y}_{\alpha}\right|^{L}+\left[\bar{\rho} x^{d} \widetilde{Y_{\alpha}^{\prime \prime} u_{x}^{\prime \prime}}\right]^{L},\right.}
\end{gathered}
$$

and interpreted as an "acoustic" solver. The second step can be obtained by inserting the first step solution in equations (3.30) and (3.31). This decomposition reflects that equation (2.11) is a diagnostic (one-way coupled) equation of the system of interest.

Remark 3.9. The Riemann invariants have been expressed as functions of the stationary states. 


\subsubsection{Analysis of the non-conservative products}

Based on a particular solution of the Riemann problem, made explicit in Proposition 3.6, and taking advantage of the LD nature of the system, we can propose an analysis of the non-conservative products. First, from the following proposition, either $\widehat{\mathbf{u}^{\prime \prime} \otimes \mathbf{u}^{\prime \prime}}$ or $\widetilde{Y}_{\alpha}$ is smooth across each wave of the Riemann problem.

Proposition 3.10. $\forall \alpha \in N_{m}, \widetilde{Y}_{\alpha}$ is constant across the central wave having material speed $\widetilde{U}$. Moreover, the tensor Reynolds, $\widetilde{\mathbf{u}^{\prime \prime} \otimes \mathbf{u}^{\prime \prime}}$, is constant across the extremal waves having speed $\widetilde{U}+\lambda_{ \pm}$.

Owing to Proposition 3.10, the non-conservative product, $\widetilde{\mathbf{u}^{\prime \prime} \otimes \mathbf{u}^{\prime \prime}} \nabla \widetilde{Y}_{\alpha}$, in equation (2.10), is always defined in a weak sense for the Riemann problem. Therefore, it cannot be considered as a pathological non-conservative product. A similar argument does not hold for the other non-conservative product, $\widehat{Y_{\alpha}^{\prime \prime} \mathbf{u}^{\prime \prime}} \cdot \nabla \widetilde{Y}_{\beta}$, in equation (2.11), owing to Remark 3.11.

Remark 3.11. Both variables $\widetilde{Y_{\alpha}^{\prime \prime} \mathbf{u}^{\prime \prime}}$ and $\widetilde{Y}_{\beta}$ may be discontinuous fields across the extremal waves of the Riemann problem.

This apparent pathology can however be adressed, relying on the following proposition.

Proposition 3.12. Whatever the regularity of the variables, equations (2.10)-(2.12) can be combined as an Ordinary Differential Equation (ODE)

$$
\begin{aligned}
\frac{\mathrm{d}}{\mathrm{d} t}\left[\widetilde{Y_{\alpha}^{\prime \prime} Y_{\beta}^{\prime \prime}}-\widetilde{Y_{\alpha}^{\prime \prime} \mathbf{u}^{\prime \prime}} t \widetilde{\mathbf{u}^{\prime \prime} \otimes \mathbf{u}^{\prime \prime}}-1 \widetilde{Y_{\beta}^{\prime \prime} \mathbf{u}^{\prime \prime}}\right]= & -\left(C_{\tau}-C_{\tau o}\right) \widetilde{\omega}\left[\widetilde{Y_{\alpha}^{\prime \prime} Y_{\beta}^{\prime \prime}}-\widetilde{Y_{\alpha}^{\prime \prime} \mathbf{u}^{\prime \prime}} t \widetilde{\mathbf{u}^{\prime \prime} \otimes \mathbf{u}^{\prime \prime}}-1 \widetilde{Y_{\beta}^{\prime \prime} \mathbf{u}^{\prime \prime}}\right] \\
& +\frac{2}{3}\left(C_{1}-1\right) \widetilde{\omega} \widetilde{Y_{\alpha}^{\prime \prime} \mathbf{u}^{\prime \prime}} t \widetilde{\mathbf{u}^{\prime \prime} \otimes \mathbf{u}^{\prime \prime}}-2 \widetilde{Y_{\beta}^{\prime \prime} \mathbf{u}^{\prime \prime}} \\
& +C_{\tau o} \widetilde{\omega} \widetilde{Y_{\alpha}^{\prime \prime} \mathbf{u}^{\prime \prime}} t \widetilde{\mathbf{u}^{\prime \prime} \otimes \mathbf{u}^{\prime \prime}}-1 \widetilde{Y_{\beta}^{\prime \prime} \mathbf{u}^{\prime \prime}},
\end{aligned}
$$

The left-hand side of equation (3.37) is defined in a weak sense, provided the temporal derivative is kept Lagrangian. The hyperbolic system has been analysed through the prism of the Riemann problem, and the identified pathological non-conservative products can be recast under a well-posed formulation. The system can be viewed as "marginally non-conservative".

Remark 3.13. We do not have much theoretical result at disposal for discontinuous solutions: uniqueness of the solution, as well as realizability, have not been proven. Our analysis of non-conservative products is based on a particular realizable solution of the Riemann problem. Therefore, our approach will consists in encoding as much as possible the known theoretical results in the numerical scheme, at the discretization step, using a Godunov approach.

\subsection{The full hyperbolic-parabolic system}

In this section, we consider the additional contribution of the diffusion-like closures that were not considered in Section 3.1.

\subsubsection{Mass conservation}

Proposition 3.14. The model preserves the mass conservation relations

$$
\sum_{\alpha=1}^{N_{m}} \widetilde{Y}_{\alpha}=1, \sum_{\alpha=1}^{N_{m}} \widetilde{Y_{\alpha}^{\prime \prime} \mathbf{u}^{\prime \prime}}=0, \sum_{i=1}^{N_{c}} \widetilde{Y}_{\alpha, i}=\widetilde{Y}_{\alpha} .
$$

Proof. The mass conservation relations, $\sum_{\alpha=1}^{N_{m}} \widetilde{Y}_{\alpha}=1, \sum_{\alpha=1}^{N_{m}} \widetilde{Y_{\alpha}^{\prime \prime} \mathbf{u}^{\prime \prime}}=0$ are preserved by the equations for the material mass fractions and associated fluxes, owing to the linearity of the equations with respect to the summation over the material index $\alpha$. Similarly, the relation $\sum_{i=1}^{N_{c}} \widetilde{Y}_{\alpha, i}=\widetilde{Y}_{\alpha}$ is preserved by the equations for the mass fractions for the constituents, by summation over the material constituent index $i$. 


\subsubsection{A class of self-similar solutions, with the diffusion contribution}

Let us assume a fixed background fluid having zero mean velocity, in 1D geometry with transverse symmetry in cartesian coordinates, homogeneous turbulent frequency, homogeneous density, and isotropic Reynolds tensor across the TMZ. Under these asumptions, System (2.8)-(2.13) can be simplified. The evolution of the turbulent kinetic energy, $\widetilde{k}$, and its dissipation rate, $\widetilde{\varepsilon}$, reduce to the following $\widetilde{k}-\widetilde{\varepsilon}$ system [15]

$$
\begin{aligned}
\partial_{t} \widetilde{k}-\partial_{x}\left(C_{k} \frac{\widetilde{k}}{\widetilde{\omega}} \partial_{x} \widetilde{k}\right) & =-\widetilde{\varepsilon} \\
\partial_{t} \widetilde{\varepsilon}-\partial_{x}\left(C_{\epsilon} \frac{\widetilde{\omega}}{\widetilde{\omega}} \partial_{x} \widetilde{\varepsilon}\right) & =-C_{\varepsilon_{2}} \widetilde{\omega} \widetilde{\varepsilon}
\end{aligned}
$$

where $C_{k}=\frac{2}{3} C_{d}$. The remaining equations of our model become

$$
\begin{aligned}
& \partial_{t} \widetilde{Y}_{\alpha}-\partial_{x} \widetilde{Y_{\alpha}^{\prime \prime} u_{x}^{\prime \prime}}=0, \\
& \partial_{t} \widetilde{Y_{\alpha}^{\prime \prime} u_{x}^{\prime \prime}}+\frac{2}{3} \widetilde{k} \partial_{x} \widetilde{Y}_{\alpha}-\partial_{x}\left(C_{k} \frac{\widetilde{k}}{\widetilde{\omega}} \partial_{x} \widetilde{Y_{\alpha}^{\prime \prime} u_{x}^{\prime \prime}}\right)=-\frac{C_{\tau}}{2} \widetilde{\omega} \widetilde{Y_{\alpha}^{\prime \prime} u_{x}^{\prime \prime}} \\
& \partial_{t} \widetilde{Y_{\alpha}^{\prime \prime 2}}+2 \widetilde{Y_{\alpha}^{\prime \prime} u_{x}^{\prime \prime}} \partial_{x} \widetilde{Y}_{\alpha}-\partial_{x}\left(C_{k} \frac{\widetilde{k}}{\widetilde{\omega}} \widetilde{\partial_{x}} \widetilde{Y_{\alpha}^{\prime \prime 2}}\right)=-\left(C_{\tau}-C_{\tau o}\right) \widetilde{\omega} \widetilde{Y_{\alpha}^{\prime \prime 2}}
\end{aligned}
$$

The existence of self-similar solutions to the problem of freely developing TMZ is suggested by dimensional analysis, in the absence of external dimensioning parameters. The TMZ width $\Lambda(t)$ itself can then be taken as the scale length of the problem. It means that possible self-similar solutions may have the form $\widetilde{q}=t^{\gamma_{a}} \check{q}(x / \Lambda(t))$, where $\gamma_{q}$ is an integer and $\check{q}$ is an arbitrary function. Both the time exponent $\gamma_{q}$ and the shape function $\check{q}$ depend on the considered quantity, $q$. In addition, one can assume TMZ having compact support, meaning zero-valued turbulent quantities outside the TMZ of increasing width. Under these considerations, simple profiles can be assumed, namely parabolic and linear profiles, for the turbulent quantities and mass fractions, respectively. Following closely the computation of [10], we obtain some self-similar solutions for this system

$$
\begin{aligned}
\widetilde{k}(x, t) & =\widetilde{k}_{o}\left(\frac{\widetilde{\omega}}{\widetilde{\omega}_{o}}\right)^{2(1-\theta)} P(x, t), \\
\widetilde{\varepsilon}(x, t) & =\widetilde{\varepsilon}_{o}\left(\frac{\widetilde{\omega}}{\widetilde{\omega}_{o}}\right)^{3-2 \theta} P(x, t), \\
\widetilde{Y}_{\alpha}(x, t) & =\frac{1}{2}\left(1+\frac{x}{\Lambda(t)}\right), \\
\widetilde{Y_{\alpha}^{\prime \prime} u_{x}^{\prime \prime}}(x, t) & =\left.\widetilde{Y_{\alpha}^{\prime \prime} u_{x}^{\prime \prime}}\right|_{o}\left(\frac{\widetilde{\omega}}{\widetilde{\omega}_{o}}\right)^{1-\theta} P(x, t), \\
\widetilde{Y_{\alpha}^{\prime \prime 2}}(x, t) & =\left.\widetilde{Y_{\alpha}^{\prime \prime 2}}\right|_{o} P(x, t),
\end{aligned}
$$

where

$$
\begin{aligned}
\frac{\widetilde{\omega}}{\widetilde{\omega}_{o}} & =\frac{1}{1+C_{\omega} \widetilde{\omega}_{o} t}, \\
P(x, t) & =\max \left[1-\left(\frac{x}{\Lambda(t)}\right)^{2}, 0\right],
\end{aligned}
$$




$$
\Lambda(t)=\Lambda_{o}\left(\frac{\widetilde{\omega}}{\widetilde{\omega}_{o}}\right)^{-\theta}
$$

The following compatibility relations must be satisfied for this self-similar solution to hold

$$
\begin{gathered}
C_{\omega}=C_{\varepsilon_{2}}-1, \quad \theta=\frac{2 C_{\omega}-1}{3 C_{\omega}}, \quad C_{k}\left[\frac{C_{\tau}}{2}+C_{\omega}(2 \theta-1)\right]=\frac{2}{3}, \quad C_{\varepsilon}=C_{k}, \\
\frac{\widetilde{k}_{o}}{\widetilde{\omega}_{o}^{2} \Lambda_{o}^{2}}=\frac{C_{\omega} \theta}{2 C_{k}}, \quad \frac{\left.\widetilde{Y_{\alpha}^{\prime \prime} u_{x}^{\prime \prime}}\right|_{o}}{\widetilde{\omega}_{0} \Lambda_{0}}=-\frac{1}{4} C_{\omega} \theta,\left.\quad \widetilde{Y_{\alpha}^{\prime \prime 2}}\right|_{o}=\frac{C_{\omega} \theta}{4\left(C_{\tau}-C_{\tau o}+C_{\omega} \theta\right)} .
\end{gathered}
$$

Finally, all the above discussion may be summarized in the following result:

Proposition 3.15. The functions defined by (3.41)-(3.45) are self-similar solutions of the RSM (2.8)-(2.13).

Remark 3.16. Physically acceptable self-similar solutions should involve both decreasing turbulent kinetic energy, $\widetilde{k}$, and turbulent frequency, $\widetilde{\omega}$. Only positive values of $C_{\omega}$ are then allowed. Finally, $C_{\omega} \geq 0$ implies that $0 \leq \theta \leq 2 / 3$.

Remark 3.17. These self-similar solution are representative of the late-time evolution of a TMZ. Analogous solutions can be found in the literature $[3,10]$. The stability of these solutions, of great interest, has not been studied here, as it is beyond the scope of this work. See [18], for instance, for examples of such stability analysis led on simpler RSMs.

\subsubsection{Filiation with other RSMs}

Proposition 3.18. Let us assume that the material mass fraction satisfies the following equation

$$
\bar{\rho} \frac{\mathrm{d} \widetilde{Y}_{\alpha}}{\mathrm{d} t}-\nabla \cdot\left[C_{c} \bar{\rho} \frac{\widetilde{\mathbf{u}^{\prime \prime} \otimes \mathbf{u}^{\prime \prime}}}{\widetilde{\omega}} \nabla \widetilde{Y}_{\alpha}\right]=0 .
$$

If $C_{c}=C_{d}$, the system (3.39)-(3.40)-(3.46) admits the self-similar solutions (3.41)-(3.42)-(3.43).

Remark 3.19. Proposition 3.18 shows that these self-similar solutions establish a connection between the second-order RSM (2.8)-(2.13) and the GSG and BHR RSMs [2,19]. The latter indeed rely on equation (3.46), for which the material mass fraction flux, $\widetilde{Y_{\alpha}^{\prime \prime} \mathbf{u}^{\prime \prime}}$, is closed at first-order instead of being computed.

\section{NUMERICAL ANALYSIS IN 1D CURVILINEAR COORDINATES}

We present, in this section, a first-order numerical scheme that maintains, at the discrete level, the main properties of the model (3.3)-(3.8), namely mass conservation, realizability, preservation of stationary states, and non-increasing entropies. This scheme is also able to handle correctly the non-conservative products, because it relies on a reformulation of the hyperbolic model (3.3)-(3.8) with equation (3.37). The proposed scheme can be viewed as an approximate Godunov scheme. As a preambular statement, let us notice that schemes having collocated components of the correlation tensor are good potential candidates to obtain the discrete realizability. Moreover, locally non-increasing discrete entropies can be obtained from collocated scheme, see [9, 13, 25] for Euler hydrodynamics. 


\subsection{Preliminaries: notations at the discrete level}

Let us consider a space domain $\Omega=\cup_{j \in J} \Omega_{j}$, paved with cells. The volume of the $j$ th cell $\Omega_{j}$ of a given set of cells $J$, is denoted by $\left|\Omega_{j}\right|$. Given a $1 \mathrm{D}$ geometry along the unit vector $\mathbf{e}_{x}$, each cell $\Omega_{j}$ is an interval $\left[x_{j-1 / 2} ; x_{j+1 / 2}\right]$ where $x_{j-1 / 2}, x_{j+1 / 2}$ are the position of its nodes. The position of the cell centroid is denoted by $x_{j}$. We introduce the vectors $\mathbf{C}_{j r}=\partial_{x_{r}}\left|\Omega_{j}\right|=x_{r}^{d} \mathbf{n}_{j r}$, where $\mathbf{n}_{j r}=n_{j r} \mathbf{e}_{x}$ stands for the external normal of $\Omega_{j}$ at its boundary $x_{r}$, giving $n_{j r}=-1$ if $r=j-1 / 2$, and $n_{j r}=1$ if $r=j+1 / 2$. Thus we obtain

$$
\sum_{r \in N_{j}} \mathbf{n}_{j r}=0, \quad \sum_{j \in M_{r}} \mathbf{C}_{j r}=0,
$$

where $N_{j}=\left\{j-\frac{1}{2}, j+\frac{1}{2}\right\}$ is the set of node indexes that are located at the boundaries of $\Omega_{j}$, while $M_{r}$ is the set of cells sharing the node with index $r$.

\subsection{A splitting approach}

We propose a splitting scheme to solve equations (3.3)-(3.8), during $\Delta t=t^{(n+1)}-t^{(n)}$. The left-hand side of this system is first evolved from an initial time, labelled with $(n)$, to an intermediate diamond state, labelled with $\diamond$, during the time step $\Delta t$. The remaining contributions, namely the dissipation and redistribution contributions, are then evolved from the diamond state, to a final state, labelled with $(n+1)$, during the time step $\Delta t$. Note that stationary states do not prevent a priori this splitting approach, as they do not involve a balance between terms located from both sides of equations (3.3)-(3.8).

The discretization leading to the diamond state is described from Sections 4.3 to 4.10 , while the discretization leading to the dissipation and redistribution contributions is described in Section 4.11.

\subsection{An approximate Godunov scheme for the mass fractions and their fluxes}

The mass fractions and their fluxes evolve according to equations (2.8)-(2.10). The resulting sub-system stands as a non-conservative sub-system of our model, whose non-conservative product, $\widetilde{\mathbf{u}^{\prime \prime} \otimes \mathbf{u}^{\prime \prime} \nabla \widetilde{Y}_{\alpha}}$, can be analysed with the help of the Riemann invariants. Therefore, a Godunov-type scheme is a good candidate for its discretization. Having the knowledge of the stationary states of the model, we propose to introduce such a scheme for the variables $\mathcal{U}_{\alpha}=\left(\widetilde{Y}_{\alpha}, \bar{\rho} x^{d} \widetilde{Y_{\alpha} \mathbf{u}^{\prime \prime}}\right)^{t}$, which are exactly the variables that can become stationary. We will show later on that this choice does lead to the discrete preservation of stationary states. We also discard the dissipation terms in the mass fraction flux equation. We can do so safely because stationary states indeed occur for a null dissipation contribution. These dissipation terms will be reintroduced later on.

Let then $\mathcal{U}_{\alpha}^{\mathcal{R}}\left(m / t, \mathcal{U}_{\alpha}^{L}, \mathcal{U}_{\alpha}^{R}\right)$ be the solution of the Riemann problem in mass coordinates, following [26]. The weak solution of the Cauchy problem, built from the initial piecewise constant data $\mathcal{U}_{\alpha}^{h}(m, t)=\mathcal{U}_{\alpha}^{h}\left(m_{j}, t^{(n)}\right)=\left.\mathcal{U}_{\alpha}\right|_{j} ^{(n)}, \forall m \in\left[m_{j-1 / 2}, m_{j+1 / 2}\right]$, can be expressed as $\mathcal{U}_{\alpha}^{h}\left(m, t+t^{(n)}\right)=$ $\mathcal{U}_{\alpha}^{\mathcal{R}}\left(\left(m-m_{j+1 / 2}\right) / t,\left.\mathcal{U}_{\alpha}\right|_{j} ^{(n)},\left.\mathcal{U}_{\alpha}\right|_{j+1} ^{(n)}\right), \forall m \in\left[m_{j-1 / 2}, m_{j+1 / 2}\right]$. The projection of this solution on piecewise constant function writes

$$
\begin{aligned}
\left.\mathcal{U}_{\alpha}\right|_{j} ^{\diamond}= & \frac{1}{\Delta m_{j}} \int_{m_{j-1 / 2}}^{m_{j}} \mathcal{U}_{\alpha}^{\mathcal{R}}\left(\left(m-m_{j-1 / 2}\right) / \Delta t,\left.\mathcal{U}_{\alpha}\right|_{j-1} ^{(n)},\left.\mathcal{U}_{\alpha}\right|_{j} ^{(n)}\right) \mathrm{d} m \\
& +\frac{1}{\Delta m_{j}} \int_{m_{j}}^{m_{j+1 / 2}} \mathcal{U}_{\alpha}^{\mathcal{R}}\left(\left(m-m_{j+1 / 2}\right) / \Delta t,\left.\mathcal{U}_{\alpha}\right|_{j} ^{(n)},\left.\mathcal{U}_{\alpha}\right|_{j+1} ^{(n)}\right) \mathrm{d} m,
\end{aligned}
$$

with the choice $\Delta m_{j}=\bar{\rho}_{j}\left|\Omega_{j}\right|$. Inserting the solution of the Riemann problem and linearizing the characteristic curves as straight lines (required for $d>1$ ) yields

$$
\left.\mathcal{U}_{\alpha}\right|_{j} ^{\diamond}=\left.\mathcal{U}_{\alpha}\right|_{j} ^{(n)}\left(1-2 \lambda_{j}^{(n)} \frac{\Delta t}{\Delta m_{j}}\right)+\lambda_{j}^{(n)} \frac{\Delta t}{\Delta m_{j}}\left(\left.\mathcal{U}_{\alpha}\right|_{j+1 / 2} ^{* L}+\left.\mathcal{U}_{\alpha}\right|_{j-1 / 2} ^{*} R\right.
$$


where $\lambda_{j}^{(n)}=\bar{\rho}_{j}^{(n)} \bar{x}_{j}^{d} \sqrt{\left.\widetilde{u_{x}^{\prime \prime 2}}\right|_{j} ^{(n)}}$, and $\bar{x}_{j}^{d} \equiv \frac{\left|\Omega_{j}\right|}{\Delta x_{j}}$. We then obtain an approximate, first-order Godunov scheme. The explicit expressions of the intermediate states, $\left.\mathcal{U}_{\alpha}\right|_{j+1 / 2} ^{*} L$ and $\left.\mathcal{U}_{\alpha}\right|_{j-1 / 2} ^{*}$, can be obtained from the continuity of some well-chosen Riemann invariants across each wave exiting or entering the cell $\Omega_{j}$.

Lemma 4.1. Let us consider the Riemann problem at node having position $x_{j+1 / 2}$, located at a boundary of $\Omega_{j}$. Combining equations (3.25) and (3.29) gives

$$
\left.\lambda_{j}^{(n)} \widetilde{Y}_{\alpha}\right|_{j+1 / 2} ^{* L}+\left[\bar{\rho} x^{d} \widehat{Y_{\alpha}^{\prime \prime} u_{x}^{\prime \prime}}\right]_{j+1 / 2}^{* L}=\left.\lambda_{j}^{(n)} \widetilde{Y}_{\alpha}\right|_{j} ^{(n)}+\left[\bar{\rho} x^{d} \widehat{Y_{\alpha}^{\prime \prime} u_{x}^{\prime \prime}}\right]_{j}^{(n)} .
$$

Remark 4.2. Equation (4.3) can be obtained by expressing the continuity of Riemann invariants either across the wave entering $\Omega_{j}$, at the position $x_{j+1 / 2}$, or across the wave exiting $\Omega_{j}$, at the position $x_{j-1 / 2}$.

Lemma 4.3. Let us consider the Riemann problem at node having position $x_{j-1 / 2}$, located at a boundary of $\Omega_{j}$. Combining equations (3.24) and (3.28) gives

$$
\left.\lambda_{j}^{(n)} \widetilde{Y}_{\alpha}\right|_{j-1 / 2} ^{* R}-\left[\bar{\rho} x^{d} \widehat{Y_{\alpha}^{\prime \prime} u_{x}^{\prime \prime}}\right]_{j-1 / 2}^{* R}=\left.\lambda_{j}^{(n)} \widetilde{Y}_{\alpha}\right|_{j} ^{(n)}-\left[\bar{\rho} x^{d} \widehat{Y_{\alpha}^{\prime \prime} u_{x}^{\prime \prime}}\right]_{j}^{(n)} .
$$

Remark 4.4. Equation (4.4) can be obtained by expressing the continuity of Riemann invariants either across the wave entering $\Omega_{j}$, at the position $x_{j-1 / 2}$, or across the wave exiting $\Omega_{j}$, at the position $x_{j+1 / 2}$.

Lemma 4.5. Let us consider the Riemann problem at node having position $x_{j-1 / 2}$, located at the other boundary of $\Omega_{j}$. Equations (3.26) and (3.27) can be rewritten as

$$
\begin{aligned}
& {\left[\bar{\rho} x^{d} \widetilde{Y_{\alpha}^{\prime \prime} u_{x}^{\prime \prime}}\right]_{j-1 / 2}^{*} R }=\left[\bar{\rho} x^{d} \widetilde{Y_{\alpha}^{\prime \prime} u_{x}^{\prime \prime}}\right]_{j-1 / 2}^{*}=\left[\bar{\rho} x^{d} \widetilde{Y_{\alpha}^{\prime \prime} u_{x}^{\prime \prime}}\right]_{j-1 / 2}^{*}, \\
&\left.\tilde{Y}_{\alpha}\right|_{j-1 / 2} ^{*} R=\left.\widetilde{Y}_{\alpha}\right|_{j-1 / 2} ^{*}=\left.\widetilde{Y}_{\alpha}\right|_{j-1 / 2} ^{*} .
\end{aligned}
$$

The following proposition is then obtained from Lemmas 4.1, 4.3 and 4.5.

Proposition 4.6. System (4.4)-(4.6) can be written as a nodal solver. At node having index $j-1 / 2$, this yields

$$
\begin{aligned}
{\left[\bar{\rho} x d \widetilde{Y_{\alpha}^{\prime \prime} u_{x}^{\prime \prime}}\right]_{j-1 / 2}^{*} } & =\frac{\lambda_{j}^{(n)}\left[\bar{\rho} x^{d} \widetilde{Y_{\alpha}^{\prime \prime} u_{x}^{\prime \prime}}\right]_{j-1}^{(n)}+\lambda_{j-1}^{(n)}\left[\bar{\rho} x^{d} \widehat{Y_{\alpha}^{\prime \prime} u_{x}^{\prime \prime}}\right]_{j}^{(n)}-\lambda_{j}^{(n)} \lambda_{j-1}^{(n)} \frac{\left.\widetilde{Y}_{\alpha}\right|_{j} ^{(n)}-\left.\widetilde{Y}_{\alpha}\right|_{j-1} ^{(n)}}{\lambda_{j}^{(n)}+\lambda_{j-1}^{(n)}},}{\left.\widetilde{Y}_{\alpha}\right|_{j-1 / 2} ^{*}}=\frac{\left.\lambda_{j}^{(n)} \widetilde{Y}_{\alpha}\right|_{j} ^{(n)}+\left.\lambda_{j-1}^{(n)} \widetilde{Y}_{\alpha}\right|_{j-1} ^{(n)}}{\lambda_{j}^{(n)}+\lambda_{j-1}^{(n)}}-\frac{\left[\bar{\rho} x^{d} \widetilde{Y_{\alpha}^{\prime \prime} u_{x}^{\prime \prime}}\right]_{j}^{(n)}-\left[\bar{\rho} x^{d} \widetilde{Y_{\alpha}^{\prime \prime} u_{x}^{\prime \prime}}\right]_{j-1}^{(n)}}{\lambda_{j}^{(n)}+\lambda_{j-1}^{(n)}} .
\end{aligned}
$$

Finally, a quadrature formula is chosen to relate $\widetilde{Y_{\alpha}^{\prime \prime} u_{x}^{\prime \prime}}$ and $\bar{\rho} x^{d} \widetilde{Y_{\alpha}^{\prime \prime} u_{x}^{\prime \prime}}$, so as to deduce a discrete equation for $\widetilde{Y_{\alpha}^{\prime \prime} u_{x}^{\prime \prime}} \diamond$, based on equation $(4.2)$. We choose

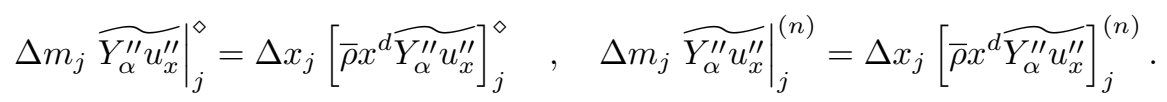

Remark 4.7. The resulting non-conservative, discrete equations for the Riemann invariants of the extremal waves are

$$
\begin{aligned}
& \bar{\rho}\left|\Omega_{j}\right|\left[\frac{\left.\mathcal{I}_{\alpha+}\right|_{j} ^{\diamond}-\left.\mathcal{I}_{\alpha+}\right|_{j} ^{(n)}}{\Delta t}\right]+\lambda_{j}^{(n)}\left(\left.\mathcal{I}_{\alpha+}\right|_{j} ^{(n)}-\left.\mathcal{I}_{\alpha+}\right|_{j-1} ^{(n)}\right)+\left(\lambda_{j}^{(n)}-\lambda_{j-1}^{(n)}\right) \frac{\lambda_{j}^{(n)}}{\lambda_{j}^{(n)}+\lambda_{j-1}^{(n)}}\left(\left.\mathcal{I}_{\alpha+}\right|_{j-1} ^{(n)}-\left.\mathcal{I}_{\alpha-}\right|_{j} ^{(n)}\right)=0, \\
& \bar{\rho}\left|\Omega_{j}\right|\left[\frac{\left.\mathcal{I}_{\alpha-\mid}\right|_{j} ^{\diamond}-\left.\mathcal{I}_{\alpha-}\right|_{j} ^{(n)}}{\Delta t}\right]-\lambda_{j}^{(n)}\left(\left.\mathcal{I}_{\alpha-}\right|_{j+1} ^{(n)}-\left.\mathcal{I}_{\alpha-}\right|_{j} ^{(n)}\right)+\left(\lambda_{j+1}^{(n)}-\lambda_{j}^{(n)}\right) \frac{\lambda_{j}^{(n)}}{\lambda_{j}^{(n)}+\lambda_{j+1}^{(n)}}\left(\left.\mathcal{I}_{\alpha+}\right|_{j} ^{(n)}-\left.\mathcal{I}_{\alpha-\mid}\right|_{j+1} ^{(n)}\right)=0 .
\end{aligned}
$$




\subsection{A scheme for the mass fraction covariances that handles with non-conservative products}

The discretization of the mass fraction covariance equation relies on the ODE (3.37). Discarding the dissipation terms in the right-hand side, that will be reintroduced later on, we propose the following discretization

$$
\left.\widetilde{Y_{\alpha}^{\prime \prime} Y_{\beta}^{\prime \prime}}\right|_{j} ^{\diamond}-\left.\left.\widetilde{Y_{\alpha}^{\prime \prime} \mathbf{u}^{\prime \prime}}\right|_{j} ^{\diamond t}\left(\left.\widetilde{\mathbf{u}^{\prime \prime} \otimes \mathbf{u}^{\prime \prime}}\right|_{j} ^{\diamond}\right)^{-1} \widetilde{Y_{\beta}^{\prime \prime} \mathbf{u}^{\prime \prime}}\right|_{j} ^{\diamond}=\left.\widetilde{Y_{\alpha}^{\prime \prime} Y_{\beta}^{\prime \prime}}\right|_{j} ^{(n)}-\left.\left.\widetilde{Y_{\alpha}^{\prime \prime} \mathbf{u}^{\prime \prime}}\right|_{j} ^{(n) t}\left(\left.\widetilde{\mathbf{u}^{\prime \prime} \otimes \mathbf{u}^{\prime \prime}}\right|_{j} ^{(n)}\right)^{-1} \widetilde{Y_{\beta}^{\prime \prime} \mathbf{u}^{\prime \prime}}\right|_{j} ^{(n)},
$$

Remark 4.8. Because both variables, $\widetilde{Y_{\alpha}^{\prime \prime} u_{x}^{\prime \prime}}$ and $\widetilde{Y}_{\beta}$, can jump across the extremal waves, the non-conservative product $\widehat{Y_{\alpha}^{\prime \prime} u_{x}^{\prime \prime}} \partial_{x} \widetilde{Y}_{\beta}$ is not straightforwardly defined in a weak sense as such. The mass fraction covariance equation expressed in the primary variables is ambiguous in this respect. The ODE (3.37) restores back a weak sense, and thus controls the non-conservative product, as explained in Section 3.1.6,

Remark 4.9. The discretization (4.10) can be reformulated in terms of discrete entropy variations, as

$$
\left.\eta_{\alpha}^{a}\right|_{j} ^{\diamond}-\left.\eta_{\alpha}^{a}\right|_{j} ^{(n)}=\left.\eta_{\alpha}^{b}\right|_{j} ^{\diamond}-\left.\eta_{\alpha}^{b}\right|_{j} ^{(n)}
$$

\subsection{A conservative, realizable and entropy scheme}

Proposition 4.10. The approximate Godunov scheme (4.2)-(4.9) gives a discrete equation for the material mass fractions that can be recast under its conservative form

$$
\left.\widetilde{Y}_{\alpha}\right|_{j} ^{\diamond}=\left.\widetilde{Y}_{\alpha}\right|_{j} ^{(n)}-\frac{\Delta t}{\bar{\rho}_{j}\left|\Omega_{j}\right|} \sum_{r \in N_{j}}\left[\bar{\rho} x^{d} \widetilde{Y_{\alpha}^{\prime \prime} u_{x}^{\prime \prime}}\right]_{r}^{*} n_{j r} .
$$

Proposition 4.11. The approximate Godunov scheme (4.2)-(4.10) satisfies unconditional discrete realizability.

Proof. The discrete equations for the correlation tensor components can be gathered in the tensorial expression

$$
\mathbb{X}_{j}^{\diamond}=\left(\mathbb{I}+\left.\Delta t \mathbb{G}_{p}\right|_{j} ^{(n)}\right) \mathbb{X}_{j}^{(n)}\left(\mathbb{I}+\left.\Delta t \mathbb{G}_{p}\right|_{j} ^{(n)}\right)^{t} \geq 0,
$$

where $\left.\mathbb{G}_{p}\right|_{j} ^{(n)} \equiv\left[\begin{array}{ll}\mathbf{0} & \mathbf{0} \\ \left.G_{p}\right|_{j} ^{(n)} & \mathbf{0}\end{array}\right]$, and $\left.G_{p}\right|_{j} ^{(n)} \equiv\left(\begin{array}{c}-\left.\frac{1}{\Delta x_{j}} \sum_{r \in N_{j}} \widetilde{Y}_{\alpha}\right|_{r} ^{*} n_{j r}^{t} \\ -\left.\frac{1}{\Delta x_{j}} \sum_{r \in N_{j}} \widetilde{Y}_{\beta}\right|_{r} ^{*} n_{j r}^{t} \\ \vdots\end{array}\right)$.

Remark 4.12. The discrete tensorial formulation (4.13) can be identified with the continuous tensorial equation (3.2), provided the resolvent matrix, $\mathbb{K}$, is approximated with a first-order Taylor expansion in the time variable: $\mathbb{K}=\mathbb{I}+\Delta t \mathbb{G}_{p}+\mathcal{O}\left(\Delta t^{2}\right)$. Our scheme simply brings an additional, unique prescription for a space discretization of the mass fraction gradients, $\left.G_{p}\right|_{j} ^{(n)}$, in the turbulent double correlation discrete equations.

Proposition 4.13. Under the stability condition $\Delta t \sqrt{\left.\widetilde{u_{x}^{\prime \prime 2}}\right|_{j} ^{(n)}} / \Delta x_{j} \leq 1$, the first-order scheme (4.2)-(4.10) satisfies the following discrete entropy inequalities

$$
\bar{\rho}_{j}\left|\Omega_{j}\right| \frac{\left.\eta_{\alpha}^{a, b}\right|_{j} ^{\diamond}-\left.\eta_{\alpha}^{a, b}\right|_{j} ^{(n)}}{\Delta t}+\left.\sum_{r \in N_{j}}\left[\bar{\rho} x^{d} \widehat{Y_{\alpha}^{\prime \prime} u_{x}^{\prime \prime}}\right]_{r}^{*} \widetilde{Y}_{\alpha}\right|_{r} ^{*} n_{j r} \leq 0
$$

where both discrete entropies have the same dissipation rate. 
Proof. Relying on equation (4.11), we can only focus on $\eta_{\alpha}^{b}$, and establish its discrete entropy inequality. Equation (4.12) can be combined with equations (4.4)-(4.6) (the discrete Riemann invariant relations), to give

$$
\begin{aligned}
\frac{\bar{\rho}_{j}\left|\Omega_{j}\right|}{2 \Delta t}\left[\left(\left.\widetilde{Y}_{\alpha}\right|_{j} ^{\diamond}\right)^{2}-\left(\left.\widetilde{Y}_{\alpha}\right|_{j} ^{(n)}\right)^{2}\right]= & -\left[\left.\sum_{r \in N_{j}} \widetilde{Y}_{\alpha}\right|_{j} ^{(n)}\left[\bar{\rho} x^{d} \widetilde{Y_{\alpha}^{\prime \prime} u_{x}^{\prime \prime}}\right]_{r}^{*} n_{j r}\right] \\
& +\frac{\bar{\rho}_{j}\left|\Omega_{j}\right|}{2 \Delta x_{j}} \frac{\left.\Delta t \widetilde{u_{x}^{\prime \prime 2}}\right|_{j} ^{(n)}}{\left|\Delta x_{j}\right|}\left[\sum_{r \in N_{j}}\left(\left.\widetilde{Y}_{\alpha}\right|_{r} ^{*}-\left.\widetilde{Y}_{\alpha}\right|_{j} ^{(n)}\right)\right]^{2} .
\end{aligned}
$$

Next, rewritting component-wise the tensorial equation (4.13), we obtain the discrete equation for $\widetilde{Y_{\alpha}^{\prime \prime} \mathbf{u}^{\prime \prime}}$. The latter can be rearranged as

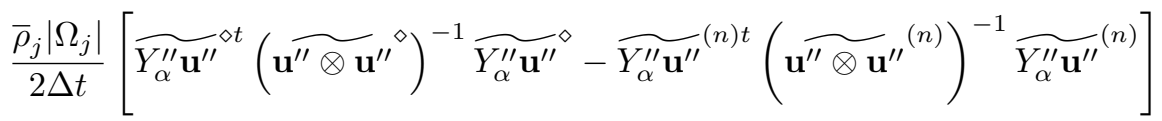

$$
\begin{aligned}
& =-\left[\left.\sum_{r \in N_{j}} \widetilde{Y}_{\alpha}\right|_{r} ^{*}\left[\bar{\rho} x^{d} \widetilde{Y_{\alpha}^{\prime \prime} u_{x}^{\prime \prime}}\right]_{j}^{(n)} n_{j r}\right]+\frac{\bar{\rho}_{j}\left|\Omega_{j}\right|}{2 \Delta x_{j}} \frac{\left.\Delta t \widetilde{u_{x}^{\prime \prime 2}}\right|_{j} ^{(n)}}{\left|\Delta x_{j}\right|}\left[\sum_{r \in N_{j}}\left(\left.\widetilde{Y}_{\alpha}\right|_{r} ^{*}-\left.\widetilde{Y}_{\alpha}\right|_{j} ^{(n)}\right) n_{j r}\right]^{2} .
\end{aligned}
$$

Summing equations (4.15) and (4.16), and noticing that

$$
\left[\sum_{r \in N_{j}}\left(\left.\tilde{Y}_{\alpha}\right|_{r} ^{*}-\left.\tilde{Y}_{\alpha}\right|_{j} ^{(n)}\right)\right]^{2}+\left[\sum_{r \in N_{j}}\left(\left.\tilde{Y}_{\alpha}\right|_{r} ^{*}-\left.\tilde{Y}_{\alpha}\right|_{j} ^{(n)}\right) n_{j r}\right]^{2}=2 \sum_{r \in N_{j}}\left(\left.\tilde{Y}_{\alpha}\right|_{r} ^{*}-\left.\tilde{Y}_{\alpha}\right|_{j} ^{(n)}\right)^{2},
$$

gives

$$
\begin{aligned}
\bar{\rho}_{j}\left|\Omega_{j}\right| \frac{\left.\eta_{\alpha}^{b}\right|_{j} ^{\diamond}-\left.\eta_{\alpha}^{b}\right|_{j} ^{(n)}}{\Delta t}= & \frac{\bar{\rho}_{j}\left|\Omega_{j}\right|}{\Delta x_{j}} \frac{\left.\Delta t \widetilde{u_{x}^{\prime \prime 2}}\right|_{j} ^{(n)}}{\left|\Delta x_{j}\right|} \sum_{r \in N_{j}}\left(\left.\widetilde{Y}_{\alpha}\right|_{r} ^{*}-\left.\widetilde{Y}_{\alpha}\right|_{j} ^{(n)}\right)^{2} \\
& -\left[\sum_{r \in N_{j}}\left(\left.\widetilde{Y_{\alpha}}\right|_{j} ^{(n)}-\left.\widetilde{Y}_{\alpha}\right|_{r} ^{*}\right)\left(\left[\bar{\rho} x^{d} \widetilde{Y_{\alpha}^{\prime \prime} u_{x}^{\prime \prime}}\right]_{r}^{*}-\left[\bar{\rho} x^{d} \widetilde{Y_{\alpha}^{\prime \prime} u_{x}^{\prime \prime}}\right]_{j}^{(n)}\right) n_{j r}\right] \\
& -\left.\sum_{r \in N_{j}}\left[\bar{\rho} x^{d} \widetilde{Y_{\alpha}^{\prime \prime} u_{x}^{\prime \prime}}\right]_{r}^{*} \widetilde{Y}_{\alpha}\right|_{r} ^{*} n_{j r} .
\end{aligned}
$$

The discrete Riemann invariant relations (4.4)-(4.6) can then be inserted in the second term of the right-hand side, which gives

$$
\bar{\rho}_{j}\left|\Omega_{j}\right| \frac{\left.\eta_{\alpha}^{a, b}\right|_{j} ^{\diamond}-\left.\eta_{\alpha}^{a, b}\right|_{j} ^{(n)}}{\Delta t}+\left.\sum_{r \in N_{j}}\left[\bar{\rho} x^{d} \widetilde{Y_{\alpha}^{\prime \prime} u_{x}^{\prime \prime}}\right]_{r}^{*} \widetilde{Y}_{\alpha}\right|_{r} ^{*} n_{j r}=\mathcal{D}_{\alpha, j}
$$

where

$$
\mathcal{D}_{\alpha, j}=\frac{\bar{\rho}_{j}\left|\Omega_{j}\right|}{\Delta x_{j}} \sqrt{\left.\widetilde{u_{x}^{\prime \prime 2}}\right|_{j} ^{(n)}}\left(\frac{\Delta t \sqrt{\left.\widetilde{u_{x}^{\prime \prime 2}}\right|_{j} ^{(n)}}}{\Delta x_{j}}-1\right) \sum_{r \in N_{j}}\left(\left.\widetilde{Y}_{\alpha}\right|_{r} ^{*}-\left.\widetilde{Y}_{\alpha}\right|_{j} ^{(n)}\right)^{2} .
$$

Enforcing inequality $\mathcal{D}_{\alpha, j} \leq 0$ yields the stability condition. 
Remark 4.14. We observe that the first-order scheme is isentropic at the discrete level, for both entropies, in the very special case of uniform mesh and constant in space Reynolds tensor, with $\Delta t=\Delta x /{\sqrt{u_{x}^{\prime \prime 2}(n)}}_{\text {. }}^{\text {. }}$

\subsection{A non Godunov-type scheme for the material constituents}

At the mixing zone boundary, there is at least one material mass fraction, denoted by index $\alpha$ for instance, that vanishes. This may lead to a divergent speed $\lambda_{\alpha}$, at the locations where the material mass fraction becomes close to zero. This speed is associated to the material constituents, while the speed associated to the material, $\lambda_{ \pm}$, do not diverge. The system partially degenerates at such boundary because the wave speed $\lambda_{\alpha}$ blows up. Thus it is no more hyperbolic, if the material constituent evolution is considered. We can nevertheless mitigate this point, because the Riemann invariant, $\widetilde{Y}_{\alpha, i} / \widetilde{Y}_{\alpha}$, associated to the wave speed $\lambda_{\alpha}$, has no physical significance (as it is not linked with any physical matter). Its transport at infinite speed cannot be forbidden, contrary to the transport of the constituent mass fraction $\widetilde{Y}_{\alpha, i}$. Therefore a Godunov scheme relying on the Riemann invariant $\widetilde{Y}_{\alpha, i} / \widetilde{Y}_{\alpha}$ is clearly not an appropriate choice, as the CFL condition would force the time step toward zero. We propose an alternative scheme, that can be written as

$$
\left.\widetilde{Y}_{\alpha, i}\right|_{j} ^{\diamond}=\left.\widetilde{Y}_{\alpha, i}\right|_{j} ^{(n)}-\left.\frac{\Delta t}{\bar{\rho}_{j}\left|\Omega_{j}\right|} \sum_{r \in N_{j}}\left[\bar{\rho} x^{d} \widetilde{Y_{\alpha}^{\prime \prime} u_{x}^{\prime \prime}}\right]_{r}^{*} \frac{\widetilde{Y}_{\alpha, i}}{\widetilde{Y}_{\alpha}}\right|_{r} n_{j r}
$$

where $\left.\frac{\widetilde{Y}_{\alpha, i}}{\widetilde{Y}_{\alpha}}\right|_{r}$ can be upwinded, depending on the sign of $\left.\lambda_{\alpha}\right|_{r} \equiv\left[\bar{\rho} x^{d} \widetilde{Y_{\alpha}^{\prime \prime} u_{x}^{\prime \prime}}\right]_{r}^{*} /\left.\widetilde{Y_{\alpha}}\right|_{r} ^{*}$.

Proposition 4.15. The discrete equation (4.19) is conservative. It is also entropy-stable, that is, it satisfies (4.17), (4.18).

Proof. The discrete equation (4.12) for the material mass fraction, $\widetilde{Y}_{\alpha}=\sum_{i=1}^{N_{c}} \widetilde{Y}_{\alpha, i}$, can be obtained from the discrete equation (4.19), by summing over the material constituents.

Remark 4.16. Initial uniform profiles of $\tilde{Y}_{\alpha, i} / \widetilde{Y}_{\alpha}$ remain uniform profiles, since this mass fraction ratio is a Riemann invariant. This stationarity property holds true at the discrete level also, owing to the discrete equations (4.12) and (4.19).

\subsection{Second-order extension in space}

We propose the following second-order extension in space. It relies on the reconstruction of a variable, $\varphi_{\alpha}$, which can be either $\widetilde{Y}_{\alpha}$ or $\bar{\rho} x^{d} \widehat{Y_{\alpha}^{\prime \prime} u_{x}^{\prime \prime}}$, at a node $r \in N_{j}$

$$
\left.\varphi_{\alpha}\left(x_{r}\right)\right|_{j}=\left.\varphi_{\alpha}\right|_{j}+\bar{\sigma}_{r} \sigma_{j}^{\varphi} \frac{\left.\varphi_{\alpha}\right|_{j+1}-\left.\varphi_{\alpha}\right|_{j-1}}{x_{j+1}-x_{j-1}}\left(x_{r}-x_{j}\right) \quad \forall r \in N_{j}
$$

where $\left.\sigma^{\varphi}\right|_{j}$ refers to an a priori limiter at cells, while $\bar{\sigma}_{r}$ refers to an a posteriori limiter at nodes. The a priori limiter is defined as

$$
\left.\sigma^{\varphi}\right|_{j}=\min _{\alpha}\left\{\left|\frac{x_{j+1}-x_{j-1}}{\left.\varphi_{\alpha}\right|_{j+1}-\left.\varphi_{\alpha}\right|_{j-1}}\right| \min \left\{\left|\frac{\left.\varphi_{\alpha}\right|_{j+1}-\left.\varphi_{\alpha}\right|_{j}}{x_{j+1}-x_{j}}\right| ;\left|\frac{\left.\varphi_{\alpha}\right|_{j-1}-\left.\varphi_{\alpha}\right|_{j}}{x_{j-1}-x_{j}}\right|\right\}\right\}
$$

if $\left(\left.\varphi_{\alpha}\right|_{j+1}-\left.\varphi_{\alpha}\right|_{j}\right)\left(\left.\varphi_{\alpha}\right|_{j-1}-\left.\varphi_{\alpha}\right|_{j}\right) \geq 0$, and $\left.\sigma^{\varphi}\right|_{j}=0$ otherwise.

The a posteriori limiter is set to its a priori value, $\bar{\sigma}_{r}=1$. We shall set $\bar{\sigma}_{r}=0$ only if an a posteriori entropy criterion, yet to be defined, would be violated. This reconstruction is then inserted in the expressions for the discrete Riemann invariant at nodes

$$
\left.\lambda_{j}^{(n)} \tilde{Y}_{\alpha}\right|_{j-1 / 2} ^{(*)}-\left[\bar{\rho} x^{d} \widetilde{Y_{\alpha}^{\prime \prime} u_{x}^{\prime \prime}}\right]_{j-1 / 2}^{(*)}=\left.\lambda_{j}^{(n)} \tilde{Y}_{\alpha}\left(x_{j-1 / 2}\right)\right|_{j} ^{(n)}-\left[\bar{\rho} x^{d} \widetilde{Y_{\alpha}^{\prime \prime} u_{x}^{\prime \prime}}\left(x_{j-1 / 2}\right)\right]_{j}^{(n)}
$$




$$
\left.\lambda_{j-1}^{(n)} \widetilde{Y}_{\alpha}\right|_{j-1 / 2} ^{(*)}+\left[\bar{\rho} x^{d} \widetilde{Y_{\alpha}^{\prime \prime} u_{x}^{\prime \prime}}\right]_{j-1 / 2}^{(*)}=\left.\lambda_{j-1}^{(n)} \widetilde{Y}_{\alpha}\left(x_{j-1 / 2}\right)\right|_{j-1} ^{(n)}+\left[\bar{\rho} x^{d} \widetilde{Y_{\alpha}^{\prime \prime} u_{x}^{\prime \prime}}\left(x_{j-1 / 2}\right)\right]_{j-1}^{(n)},
$$

which can be viewed as a nodal solver, whose solution is

$$
\begin{aligned}
& {\left[\bar{\rho} x^{d} \widetilde{Y_{\alpha}^{\prime \prime} u_{x}^{\prime \prime}}\right]_{j-1 / 2}^{*}=\frac{\lambda_{j}^{(n)}\left[\bar{\rho} x^{d} \widetilde{Y_{\alpha}^{\prime \prime} u_{x}^{\prime \prime}}\left(x_{j-1 / 2}\right)\right]_{j-1}^{(n)}+\lambda_{j-1}^{(n)}\left[\bar{\rho} x^{d} \widetilde{Y_{\alpha}^{\prime \prime} u_{x}^{\prime \prime}}\left(x_{j-1 / 2}\right)\right]_{j}^{(n)}}{\lambda_{j}^{(n)}+\lambda_{j-1}^{(n)}}} \\
& -\lambda_{j}^{(n)} \lambda_{j-1}^{(n)} \frac{\left.\widetilde{Y}_{\alpha}\left(x_{j-1 / 2}\right)\right|_{j} ^{(n)}-\left.\tilde{Y}_{\alpha}\left(x_{j-1 / 2}\right)\right|_{j-1} ^{(n)}}{\lambda_{j}^{(n)}+\lambda_{j-1}^{(n)}} \\
& \left.\widetilde{Y}_{\alpha}\right|_{j-1 / 2} ^{*}=\frac{\left.\lambda_{j}^{(n)} \tilde{Y}_{\alpha}\left(x_{j-1 / 2}\right)\right|_{j} ^{(n)}+\left.\lambda_{j-1}^{(n)} \tilde{Y}_{\alpha}\left(x_{j-1 / 2}\right)\right|_{j-1} ^{(n)}}{\lambda_{j}^{(n)}+\lambda_{j-1}^{(n)}} \\
& -\frac{\left[\bar{\rho} x^{d} \widetilde{Y_{\alpha}^{\prime \prime} u_{x}^{\prime \prime}}\left(x_{j-1 / 2}\right)\right]_{j}^{(n)}-\left[\bar{\rho} x^{d} \widetilde{Y_{\alpha}^{\prime \prime} u_{x}^{\prime \prime}}\left(x_{j-1 / 2}\right)\right]_{j-1}^{(n)}}{\lambda_{j}^{(n)}+\lambda_{j-1}^{(n)}} .
\end{aligned}
$$

These expressions can be inserted in the discrete equations (4.12), (4.13) and (4.19), that rely on a simple forward Euler time discretization. It could also be combined with a Runge-Kutta higher-order extension, as we shall show in the next Sections.

Proposition 4.17. The second-order a priori reconstruction preserves linear solutions, and degenerates at first-order at extrema, by construction.

Proposition 4.18. The second-order scheme preserves the stationary states and the mass conservation relations (3.38), at the discrete level, by construction.

In the following, we investigate the stability of this high-order in space extension, in relation with the time discretization.

\subsection{Runge-Kutta $L^{2}$ stabilization: a Von Neumann analysis}

The extension of the space discretization to second-order may affect stability. To evaluate its impact, a standard Von Neumann analysis is proposed for the discrete equations satisfied by the variable $\mathcal{U}_{\alpha}=\left(\widetilde{Y}_{\alpha}, \bar{\rho} x^{d} \widetilde{Y_{\alpha} \mathbf{u}^{\prime \prime}}\right)^{t}$. Strong hypotheses are made to do so. Hence, all subsequent stability constraints should be understood as conditions related to linear stability, distinct from nonlinear stability.

Proposition 4.19. Let the Reynolds tensor and mean density be constant in time and space, over an interval $\Omega=[0,2 \pi]$ in a $1 D$ geometry, with transverse symmetry in cartesian coordinates $(d=0)$. Let the dissipation and diffusion terms be discarded. Then the equation for $\mathcal{U}_{\alpha}$ reduces to the simple "acoustic" system

$$
\frac{\mathrm{d}}{\mathrm{d} t} \mathcal{U}_{\alpha}+\left(\begin{array}{cc}
0 & 1 \\
\lambda^{2} & 0
\end{array}\right) \partial_{x} \mathcal{U}_{\alpha}=0
$$

with sound speed $\lambda=\sqrt{\widetilde{u_{x}^{\prime \prime 2}}}$.

The system (4.23) admits exact solutions defined as the superposition of harmonic modes $e^{\hat{\imath}(k x-\omega t)}$, where $k$ is a wavenumber, $\omega$ is a frequency that satisfies the dispersion relation $\omega=\lambda k$, and $\hat{\imath}$ the imaginary unity, $\hat{\imath}^{2}=-1$. 
Remark 4.20. An interval of arbitrary length $\Omega=[0, L]$ can also be considered for this stability analysis. We restrict to $\Omega=[0,2 \pi]$, without loss of generality, for the sake of clarity.

Proposition 4.21. The proposed first-order discretization (4.2)-(4.10) of (4.23), as well as its unlimited extension to second-order in space (4.20)-(4.22) (limiters are set to 1), can be rewritten as $\left.\hat{\mathcal{U}}_{\alpha}\right|_{j} ^{\triangleright}=\left.A \hat{\mathcal{U}}_{\alpha}\right|_{j} ^{(n)}$, where $\hat{\mathcal{U}}_{\alpha}=\left(\begin{array}{ll}\lambda & 0 \\ 0 & 1\end{array}\right) \mathcal{U}_{\alpha}, A=I+\mu S$ is the so-called amplification matrix, $\mu=\lambda \Delta t / \Delta x$ is the Courant number,

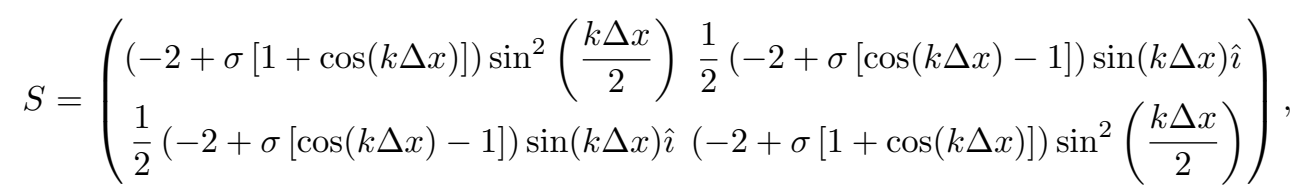

and $\sigma$ is set to 0 or 1 , respectively for the first-order or the second-order case. The spectral radius of $A$ is bounded by one, under the CFL criterion

$$
\begin{aligned}
& \left\{(2-\sigma[1+\cos (k \Delta x)]) \sin ^{2}\left(\frac{k \Delta x}{2}\right)+\frac{1}{4}\left(-2+\sigma[\cos (k \Delta x)-1]^{2}\right) \sin ^{2}(k \Delta x)\right\} \mu \\
& \leq 2(2-\sigma[1+\cos (k \Delta x)]) \sin ^{2}\left(\frac{k \Delta x}{2}\right)
\end{aligned}
$$

where

- the first-order discretization in space $(\sigma=0)$ yields: $\sin ^{2}\left(\frac{k \Delta x}{2}\right) \mu \leq \sin ^{2}\left(\frac{k \Delta x}{2}\right)$,

- the second-order discretization in space $(\sigma=1)$ yields: $[9-\cos (2 k \Delta x)] \sin ^{2}\left(\frac{k \Delta x}{2}\right) \mu \leq 8 \sin ^{4}\left(\frac{k \Delta x}{2}\right)$.

The scheme will be deemed as linearly stable, with respect to the exact harmonic solution, if all eigenvalues of $A$ are, in modulus, less than or equal to one. From Proposition 4.21, we observe that the constant mode, $k=0$, is unconditionally stable for both first-order and second-order discretization in space. Note also that the smallest wavelength, with wavenumber $k=\pi / \Delta x$, is stable under the condition $\mu \leq 1$ in either cases. The first-order scheme in space and time is $L^{2}$ stable if $\mu \leq 1$. On the contrary, the second-order in space, first-order in time scheme is unconditionally unstable. Figure 2 indeed shows the modulus of the eigenvalues (amplification factor) related to the first wavenumbers, against the Courant number. This suggests the existence, for each Courant number, of at least one wavenumber having an amplification factor greater than one.

Let us now investigate whether a higher-order time discretization might bring stabilization.

Remark 4.22. The amplification matrix $A$, if computed from the second-order, low storage Runge-Kutta time discretization of Appendix A, becomes

$$
A=I+\mu S+\frac{\mu^{2}}{2} S^{2} .
$$

Note that the Taylor series should be pursued for higher-order time discretization. At the third and fourth order, this series corresponds to the amplification matrices of the third and fourth order, low storage Runge-Kutta scheme of Appendix A.

Figure 3 shows the amplification factor of the first wavenumber, for the high-order time discretizations, up to fourth-order, against the Courant number. We observe that the stability of our scheme only depends on the stability of the $2 \Delta x$-wave, because the amplification factor from this particular wave dominates the other 


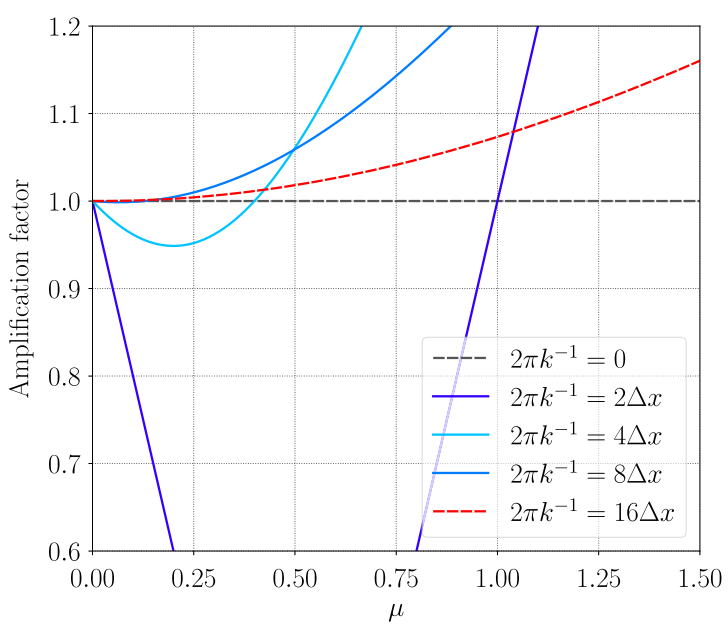

FiguRE 2. Amplification factor as a function of the Courant number, for the Euler explicit time discretization, combined with the second-order space discretization. A variety of wavenumbers is shown. The amplification factor for the null wavelength (black dashed line) is constant, while the one related to the $16 \Delta x$ wavelength (red dashed line) has a very narrow stability domain. The intermediate wavelength are represented by solid lines. Increasing the wavelength narrows the stability domains.
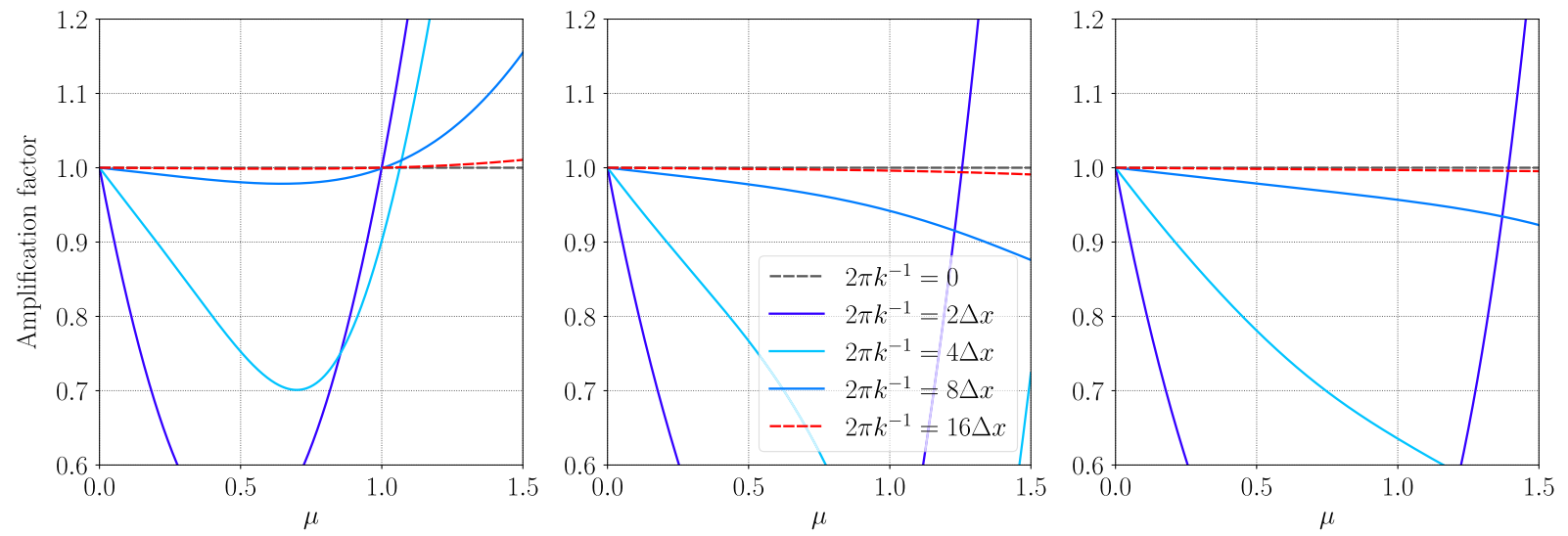

Figure 3. Same graph as in Figure 2, now related to the high order time discretization: second-order (left), third-order (center) and fourth-order (right). Increasing the wavelength now broadens the stability domains.

ones, as soon as one wave becomes unstable (its amplification factor exeeds unity). The CFL criteria are given analytically in Table 1 for the first and higher-order scheme.

As a conclusion, we have shown that a stabilization is required for the second-order in space discretization. If combined with a first-order forward Euler time discretization, it is indeed unconditionally unstable. We have also shown that high-order Runge-Kutta schemes provide the desired stabilizing effect, and that a second-order Runge-Kutta time discretization is sufficient. In the next section, we shall show how the second-order spatial discretization can be appropriately combined with such second-order Runge-Kutta time discretization, having in view an efficient and simple implementation. 
TABle 1. Maximal Courant number $\mu$ for the CFL-criterion. In this table, $\gamma=(4+\sqrt{17})^{1 / 3}$ and $\kappa=\left(\frac{43+9 \sqrt{29}}{2}\right)^{1 / 3}$.

\begin{tabular}{lllll}
\hline \hline Time order: & 1st & 2nd & 3rd & 4 th \\
\hline 1st order-space & 1 & 1 & $\left(\gamma^{2}+\gamma-1\right) / 2 \gamma$ & $\left(\kappa^{2}+2 \kappa-5\right) / 3 \kappa$ \\
2nd order-space & 0 & 1 & $\left(\gamma^{2}+\gamma-1\right) / 2 \gamma$ & $\left(\kappa^{2}+2 \kappa-5\right) / 3 \kappa$ \\
\hline
\end{tabular}

\subsection{A second proof of discrete realizability}

Lemma 4.23. Let $\mathcal{A}$ a be a symmetric matrix

$$
\mathcal{A}=\left(\begin{array}{ccccc}
a & b_{1} & b_{2} & \cdots & b_{N} \\
b_{1} & c_{11} & c_{12} & \cdots & c_{1 N} \\
b_{2} & c_{12} & c_{22} & \cdots & c_{2 N} \\
\vdots & \vdots & \vdots & & \vdots \\
b_{N} & c_{1 N} & c_{2 N} & \cdots & c_{N N}
\end{array}\right)
$$

and $D=\left(d_{i j}\right)_{1 \leq i, j \leq N}$ such as

$$
d_{i j}=\left|\begin{array}{cc}
a & b_{j} \\
b_{i} & c_{i j}
\end{array}\right|=a c_{i j}-b_{i} b_{j}=d_{j i}
$$

If $\mathcal{A}$ is Semi Definite Positive (SDP), then D is also SDP.

The proof of Lemma 4.23 is postponed to Appendix B, and can be easily extended to the case where $a$ is a matrix and $b_{i}$ a vector, using the Sylvester characterization for the SDP property.

Proposition 4.24. Let $\mathcal{U}_{\alpha}^{\diamond}$ be the intermediate state defined by equations (4.2)-(4.9). Assume that the corresponding mass fraction covariances are defined by equation (4.10). Then $\mathbb{X}^{\diamond} \geq 0$, in the sense of symmetric matrices.

Proof of Proposition 4.24. Dropping the cell index, the discrete correlation tensor

$$
\mathbb{X}^{\diamond}=\left(\begin{array}{cccc}
\widetilde{\mathbf{u}^{\prime \prime} \otimes \mathbf{u}^{\prime \prime}} & \widehat{Y_{\alpha}^{\prime \prime} \mathbf{u}^{\prime \prime}} & \widehat{Y_{\beta}^{\prime \prime} \mathbf{u}^{\prime \prime}} & \ldots \\
\widetilde{Y_{\alpha}^{\prime \prime} \mathbf{u}^{\prime \prime}} & \widehat{Y_{\alpha}^{\prime \prime 2}} & \widehat{Y_{\alpha}^{\prime \prime} Y_{\beta}^{\prime \prime}} & \ldots \\
\widetilde{Y_{\beta}^{\prime \prime} \mathbf{u}^{\prime \prime}} & \widehat{Y_{\alpha}^{\prime \prime} Y_{\beta}^{\prime \prime}} & \widehat{Y_{\beta}^{\prime \prime}}{ }^{\diamond} & \ldots \\
\vdots & \vdots & \vdots & \ddots
\end{array}\right) .
$$

can be written as

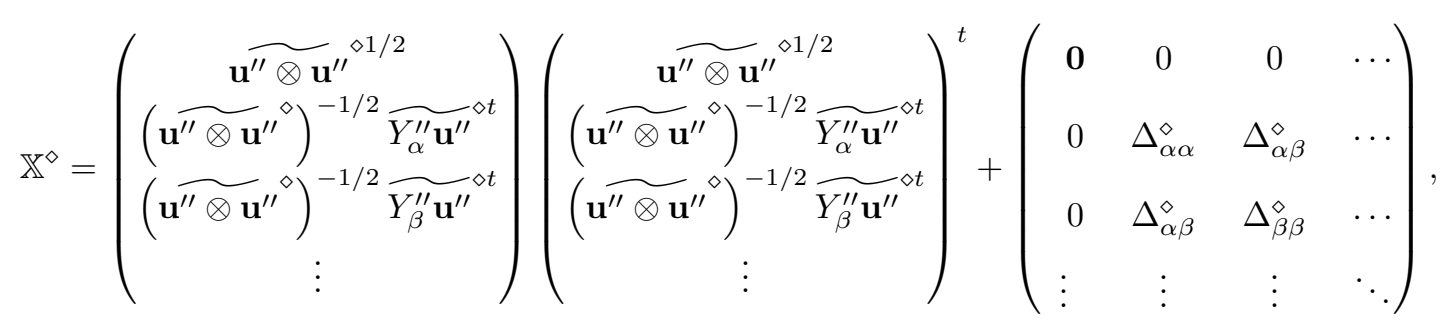


with

$$
\left.\Delta_{\alpha \beta}^{\diamond}=\widetilde{Y_{\alpha}^{\prime \prime} Y_{\beta}^{\prime \prime}}-\widetilde{Y_{\alpha}^{\prime \prime} \mathbf{u}^{\prime \prime}}{ }^{\diamond}\left(\widetilde{\mathbf{u}^{\prime \prime} \otimes \mathbf{u}^{\prime \prime}}\right)^{\diamond} \widetilde{Y_{\beta}^{\prime \prime} \mathbf{u}^{\prime \prime}}={\widetilde{Y_{\alpha}^{\prime \prime} Y_{\beta}^{\prime \prime}}}^{(n)}-{\widetilde{Y_{\alpha}^{\prime \prime} \mathbf{u}^{\prime \prime}}}^{(n)^{t}}\left(\widetilde{\mathbf{u}^{\prime \prime} \otimes \mathbf{u}^{\prime \prime}}\right)^{(n)}\right)^{-1}{\widetilde{Y_{\beta}^{\prime \prime} \mathbf{u}^{\prime \prime}}}^{(n)} .
$$

The first term of the previous equation for $\mathbb{X}^{\diamond}$ is clearly Semi Definite Positive (SDP). Therefore, requiring the second term to be SDP is a sufficient condition for $\mathbb{X}^{\diamond}$ to be also SDP. This is the case because the matrix $\Delta=\left(\Delta_{\alpha \beta}\right)$ is also SDP, according to Lemma 4.23.

In the following section, we rely on Proposition 4.24 to propose a second-order Runge-Kutta stabilization on $\mathcal{U}_{\alpha}$ only, followed by the discrete equation for the mass fraction covariances (4.10). This minimizes the required storage, while ensuring discrete realizability.

\subsection{An entropy-based a posteriori treatment}

We wish to obtain a second-order in space scheme for smooth solutions, while ensuring a nonlinear entropy stability. Recall that linear stability has already been obtained with a second-order in time Runge-Kutta scheme, which controls smooth solutions of the linearized hyperbolic system. Note that such entropy stabilization may also bring an additional control over spurious oscillations, for non-smooth solutions. To achieve so, let us investigate the modification of the first-order discrete entropy inequality (4.17), by the second-order scheme. Noticing that the discrete entropy dissipation rates are the same for both entropies $\eta^{a}$ and $\eta^{b}$, at first and also second-order, we drop $\eta^{a}$ safely and focus on $\eta^{b}$, which is related to the variable $\mathcal{U}_{\alpha}$. The explicit Euler stages, $k=1,2$, of our RK2 method, can be written

$$
\begin{array}{ll}
\left.\mathcal{U}_{\alpha}\right|_{j \in J} ^{(k=1)}=\mathcal{L}_{\mathcal{U}}\left(\left.\mathcal{U}_{\alpha}\right|_{j \in J} ^{(n)}, \Delta t\right), & \left.\widetilde{Y}_{\alpha, i}\right|_{j \in J} ^{(k=1)}=\mathcal{L} \mathcal{Y}\left(\left.\tilde{Y}_{\alpha, i}\right|_{j \in J} ^{(n)}, \Delta t\right), \\
\left.\mathcal{U}_{\alpha}\right|_{j \in J} ^{(k=2)}=\mathcal{L}_{\mathcal{U}}\left(\left.\mathcal{U}_{\alpha}\right|_{j \in J} ^{(1)}, \Delta t\right), & \left.\widetilde{Y}_{\alpha, i}\right|_{j \in J} ^{(k=2)}=\mathcal{L}_{\mathcal{Y}}\left(\left.\widetilde{Y}_{\alpha, i}\right|_{j \in J} ^{(k=1)}, \Delta t\right),
\end{array}
$$

where $\mathcal{L}_{\mathcal{U}}$ and $\mathcal{L}_{\mathcal{Y}}$ are symbolic operators refering to the first-order in time, second-order in space discretizations of Sections (4.3), (4.6) and (4.7). The final convex combination stage of our RK2 method writes, $\forall j \in J$,

$$
\begin{aligned}
\mathcal{U}_{j}^{\diamond} & =\frac{1}{2} \mathcal{U}_{j}^{(n)}+\frac{1}{2} \mathcal{U}_{j}^{(k=2)}, \\
\left.\widetilde{Y}_{\alpha, i}\right|_{j} ^{\diamond} & =\left.\frac{1}{2} \widetilde{Y}_{\alpha, i}\right|_{j} ^{(n)}+\left.\frac{1}{2} \widetilde{Y}_{\alpha, i}\right|_{j} ^{(k=2)} .
\end{aligned}
$$

This RK2 method brings the following discrete entropy increments

$$
\begin{array}{r}
\bar{\rho}_{j}\left|\Omega_{j}\right| \frac{\left.\eta_{\alpha}^{b}\right|_{j} ^{(k=1)}-\left.\eta_{\alpha}^{b}\right|_{j} ^{(n)}}{\Delta t}+\left.\sum_{r \in N_{j}}\left[\bar{\rho} x^{d} \widehat{Y_{\alpha}^{\prime \prime} u_{x}^{\prime \prime}}\right]_{r}^{(*, n)} \widetilde{Y}_{\alpha}\right|_{r} ^{(*, n)} n_{j r}=\mathcal{D}_{\alpha, j}^{(k=1)}, \\
\bar{\rho}_{j}\left|\Omega_{j}\right| \frac{\left.\eta_{\alpha}^{b}\right|_{j} ^{(k=2)}-\left.\eta_{\alpha}^{b}\right|_{j} ^{(k=1)}}{\Delta t}+\left.\sum_{r \in N_{j}}\left[\bar{\rho} x^{d} \widetilde{Y_{\alpha}^{\prime \prime} u_{x}^{\prime \prime}}\right]_{r}^{(*, k=1)} \widetilde{Y}_{\alpha}\right|_{r} ^{(*, k=1)} n_{j r}=\mathcal{D}_{\alpha, j}^{(k=2)},
\end{array}
$$

where the superscript $(*, k)$ denotes the second-order solution of the nodal solver at the Runge-Kutta stage having index $k$, and $\mathcal{D}_{\alpha, j}^{(k=1,2)}$ are the (unsigned) second-order entropy dissipation rates. The last Runge-Kutta stage to the diamond state is a convex combination stage.

Proposition 4.25. The diamond state computed from equations (4.25) to (4.27) satisfies

$$
\left.\eta_{\alpha}^{b}\right|_{j} ^{\diamond} \leq \frac{1}{2}\left(\left.\eta_{\alpha}^{b}\right|_{j} ^{(n)}+\left.\eta_{\alpha}^{b}\right|_{j} ^{(k=2)}\right)
$$


Proof.

$$
\begin{aligned}
\left.\eta_{\alpha}^{b}\right|_{j} ^{\diamond} & =\eta_{\alpha}^{b}\left(\left.\tilde{Y}_{\alpha}\right|_{j} ^{\diamond},\left.\widetilde{Y_{\alpha}^{\prime \prime} \mathbf{u}^{\prime \prime}}\right|_{j} ^{\diamond},\left.\widetilde{\mathbf{u}^{\prime \prime} \otimes \mathbf{u}^{\prime \prime}}\right|_{j} ^{\diamond}\right)=\eta_{\alpha}^{b}\left(\left.\widetilde{Y}_{\alpha}\right|_{j} ^{\diamond},\left.\widetilde{Y_{\alpha}^{\prime \prime} \mathbf{u}^{\prime \prime}}\right|_{j} ^{\diamond},\left.\widetilde{\mathbf{u}^{\prime \prime} \otimes \mathbf{u}^{\prime \prime}}\right|_{j} ^{(n)}\right) \\
& =\eta_{\alpha}^{b}\left(\left.\frac{1}{2} \tilde{Y}_{\alpha}\right|_{j} ^{(n)}+\left.\frac{1}{2} \widetilde{Y}_{\alpha}\right|_{j} ^{(k=2)},\left.\frac{1}{2} \widetilde{Y_{\alpha}^{\prime \prime} \mathbf{u}^{\prime \prime}}\right|_{j} ^{(n)}+\left.\frac{1}{2} \widetilde{Y_{\alpha}^{\prime \prime} \mathbf{u}^{\prime \prime}}\right|_{j} ^{(k=2)},\left.\widetilde{\mathbf{u}^{\prime \prime} \otimes \mathbf{u}^{\prime \prime}}\right|_{j} ^{(n)}\right) \\
& \leq \frac{1}{2} \eta_{\alpha}^{b}\left(\left.\widetilde{Y}_{\alpha}\right|_{j} ^{(n)},\left.\widetilde{Y_{\alpha}^{\prime \prime} \mathbf{u}^{\prime \prime}}\right|_{j} ^{(n)},\left.\widetilde{\mathbf{u}^{\prime \prime} \otimes \mathbf{u}^{\prime \prime}}\right|_{j} ^{(n)}\right)+\frac{1}{2} \eta_{\alpha}^{b}\left(\left.\widetilde{Y}_{\alpha}\right|_{j} ^{(k=2)},\left.\widetilde{Y_{\alpha}^{\prime \prime} \mathbf{u}^{\prime \prime}}\right|_{j} ^{(k=2)},\left.\widetilde{\mathbf{u}^{\prime \prime} \otimes \mathbf{u}^{\prime \prime}}\right|_{j} ^{(n)}\right)
\end{aligned}
$$

by convexity of the function $\eta_{\alpha}^{b}$.

From Proposition 4.25, we obtain straightforwardly $\left.\eta_{\alpha}^{b}\right|_{j} ^{\diamond}-\left.\eta_{\alpha}^{b}\right|_{j} ^{(k=2)} \leq \frac{1}{2}\left(\left.\eta_{\alpha}^{b}\right|_{j} ^{(n)}+\left.\eta_{\alpha}^{b}\right|_{j} ^{(k=2)}\right)-\left.\eta_{\alpha}^{b}\right|_{j} ^{(k=2)}$. Gathering all Runge-Kutta stages, and defining

$$
\mathcal{E}_{\alpha, l h s}^{2 \mathrm{nd}} \equiv \bar{\rho}_{j}\left|\Omega_{j}\right| \frac{\left.\eta_{\alpha}^{b}\right|_{j} ^{\diamond}-\left.\eta_{\alpha}^{b}\right|_{j} ^{(n)}}{\Delta t}+\frac{1}{2} \sum_{r \in N_{j}}\left(\left.\left[\bar{\rho} x^{d} \widehat{Y_{\alpha}^{\prime \prime} u_{x}^{\prime \prime}}\right]_{r}^{(*, n)} \widetilde{Y}_{\alpha}\right|_{r} ^{(*, n)}+\left.\left[\bar{\rho} x^{d} \widehat{Y_{\alpha}^{\prime \prime} u_{x}^{\prime \prime}}\right]_{r}^{(*, k=1)} \widetilde{Y}_{\alpha}\right|_{r} ^{(*, k=1)}\right) n_{j r}
$$

we finally obtain the second-order discrete entropy inequality

$$
\mathcal{E}_{\alpha, l h s}^{2 \mathrm{nd}} \leq \frac{1}{2}\left(\mathcal{D}_{\alpha, j}^{(k=1)}+\mathcal{D}_{\alpha, j}^{(k=2)}\right) .
$$

This latter expression gives a non-ambiguous discrete expression of the entropy flux through the Runge-Kutta stages. Now equipped with the first-order and second-order discrete entropy inequalities, respectively (4.17) and (4.29), we can step toward a discrete entropy-based a posteriori treatment for the high-order approximation of $\mathcal{U}_{\alpha}$. Relying on the isentropic nature of the Riemann problem in the absence of diffusion and dissipation, we impose, a posteriori, the left-hand side of the second-order entropy inequality $(4.29), \mathcal{E}_{\alpha, l h s}^{2 \text { nd }}$, to be bounded by the first-order discrete entropy dissipation rate, defined by equation (4.18), and denoted by $\mathcal{D}_{\alpha, j}^{(1 \mathrm{st})}$. Would this criteria be violated, then the scheme would locally reduce to first-order. The order degradation is performed following the e-MOOD approach [4], where the local reconstruction order is determined uniquely at each node, for the sake of coherence in the nodal solver, which gets the same reconstruction order from all the neighbour meshes. The reconstruction is therefore not unique for cells which have both "first-order" and "higher-order nodes". Let us define

- $J$ and $N$ the set of cell indices and nodes indices, respectively,

- $T_{j}=\left(\exists \alpha / \mathcal{E}_{\alpha, l h s}^{2 \text { nd }}>\left|\mathcal{D}_{\alpha, j}^{(1 \mathrm{st})}\right|\right)$ a boolean that locally points out the need for an order degradation,

- $D=\left\{j \in J / T_{j}\right.$ or $T_{j+1}$ or $\left.T_{j-1}\right\}$, the domain that requires degradation to first-order,

- RK2 the set of 3 stages of our second-order Runge-Kutta scheme. We have chosen a specific Runge-Kutta scheme, see Appendix A and references [17,43], so that each stage, having index $k$, and denoted by RK2 $(k)$, can be either an "Explicit Euler" stage, or a "Convex Combination" stage.

The algorithm that makes the transition from the initial state, $\left\{\left.\mathcal{U}_{\alpha}\right|_{j} ^{(n)},\left.\widetilde{Y}_{\alpha, i}\right|_{j} ^{(n)}\right\}_{j \in J}$, to the diamond state, $\left\{\left.\mathcal{U}_{\alpha}\right|_{j} ^{\diamond},\left.\widetilde{Y}_{\alpha, i}\right|_{j} ^{\diamond}\right\}_{j \in J}$, reads

(1) Initialisation:

- Constrain the time step according to the stability condition given by Proposition 4.13,

- $\forall r \in N, \forall \alpha \in N_{m}$ : compute $\left.\mathcal{U}_{\alpha}\right|_{r} ^{(*)}$, the first-order solution of the nodal solver, equations (4.7) and (4.8), - $D=\emptyset$. 
(2) High-order guess for $\left\{\left.\mathcal{U}_{\alpha}\right|_{j} ^{\diamond},\left.\widetilde{Y}_{\alpha, i}\right|_{j} ^{\diamond}\right\}_{j \in J}$ :

- Runge-Kutta stages: for increasing $k \in[1,3]$ :

If $R K 2(k)==$ "Explicit Euler":

$\forall r \in N:$

○ $\forall j \in M_{r}, \forall \alpha \in N_{m}$ : compute the nodal reconstructed state $\left.\mathcal{U}_{\alpha}\left(x_{r}\right)\right|_{j}$, according to equation (4.20),

○ $\forall \alpha \in N_{m}$ : compute $\left.\mathcal{U}_{\alpha}\right|_{r} ^{(*)}$, the second-order solution of the nodal solver, equations (4.21) and $(4.22)$,

$\forall j \in J, \forall \alpha \in N_{m}, \forall i \in N_{c}$ : apply an explicit Euler stage, with the discrete equations (4.2), (4.9) and (4.19), which gives the updated state $\left\{\left.\mathcal{U}_{\alpha}\right|_{j} ^{(k)},\left.\widetilde{Y}_{\alpha, i}\right|_{j} ^{(k)}\right\}$ from the previous Runge-Kutta state $\left\{\left.\mathcal{U}_{\alpha}\right|_{j^{\prime}} ^{(k-1)},\left.\widetilde{Y}_{\alpha, i}\right|_{j^{\prime}} ^{(k-1)}\right\}_{j^{\prime} \in J}$.

Else:

$\forall j \in J, \forall \alpha \in N_{m}, \forall i \in N_{c}$ : compute the updated state, $\left\{\left.\mathcal{U}_{\alpha}\right|_{j} ^{\diamond},\left.\widetilde{Y}_{\alpha, i}\right|_{j} ^{\diamond}\right\}$, as a convex combination between the initial state, $\left\{\left.\mathcal{U}_{\alpha}\right|_{j} ^{(n)},\left.\widetilde{Y}_{\alpha, i}\right|_{j} ^{(n)}\right\}$, and the current Runge-Kutta state, $\left\{\left.\mathcal{U}_{\alpha}\right|_{j} ^{(k)},\left.\widetilde{Y}_{\alpha, i}\right|_{j} ^{(k)}\right\}$

- $\forall j \in J$ : compute $T_{j}$. Then update $D$.

(3) A posteriori, correction loop:

- While $D \neq \emptyset$,

○ $\forall r \in N, \forall j \in M_{r}$, if $j \in D: \forall \alpha \in N_{m}$ : compute $\left.\mathcal{U}_{\alpha}\right|_{r} ^{(*)}$, the first-order solution of the nodal solver, equations (4.7) and (4.8),

$\circ \forall j \in D, \forall \alpha \in N_{m}, \forall i \in N_{c}$, apply an explicit Euler stage, with the discrete equations (4.2), (4.9) and (4.19), which gives the updated state $\left\{\left.\mathcal{U}_{\alpha}\right|_{j} ^{\diamond},\left.\widetilde{Y}_{\alpha, i}\right|_{j} ^{\diamond}\right\}$ from $\left\{\left.\mathcal{U}_{\alpha}\right|_{j^{\prime}} ^{(n)},\left.\widetilde{Y}_{\alpha, i}\right|_{j^{\prime}} ^{(n)}\right\}_{j^{\prime} \in J}$.

○ $D=\emptyset . \forall j \in J$ : compute $T_{j}, \forall j \in J$. Then update $D$.

(4) Update of the concentration covariances, $\left.\widetilde{Y_{\alpha}^{\prime \prime} Y_{\beta}^{\prime \prime}}\right|_{j \in J} ^{\diamond}$, by the discrete equation (4.10).

This algorithm always terminates. In principle, the scheme might be degraded to first order in all cells. In practice, we shall see that this situation does not occur, as the a posteriori corrections are barely activated. If nevertherless activated, they remain localized in space, for instance at discontinuities.

\subsection{A realizable discretization for the dissipation and redistribution terms}

According to the splitting of dissipation and redistribution terms, adopted in Section 4.2, the computation of the final state, at time $t^{(n+1)}$, is obtained by solving the system

$$
\begin{aligned}
& \frac{\mathrm{d}}{\mathrm{d} t} \widetilde{Y_{\alpha}^{\prime \prime} \mathbf{u}^{\prime \prime}}=-\frac{1}{2}\left(C_{\tau}+C_{1}\right) \widetilde{\omega} \widetilde{Y_{\alpha}^{\prime \prime} \mathbf{u}^{\prime \prime}}, \\
& \frac{\mathrm{d}}{\mathrm{d} t} \widetilde{Y_{\alpha}^{\prime \prime} Y_{\beta}^{\prime \prime}}=-\left(C_{\tau}-C_{\tau o}\right) \widetilde{\omega} \widetilde{Y_{\alpha}^{\prime \prime} Y_{\beta}^{\prime \prime}}, \\
& \frac{\mathrm{d}}{\mathrm{d} t} \widetilde{\mathbf{u}^{\prime \prime} \otimes \mathbf{u}^{\prime \prime}}=-\frac{2}{3} \widetilde{\omega} \widetilde{k} \mathbb{I}-C_{1} \widetilde{\omega}\left[\widetilde{\mathbf{u}^{\prime \prime} \otimes \mathbf{u}^{\prime \prime}}-\frac{2}{3} \widetilde{k} \mathbb{I}\right], \\
& \frac{\mathrm{d}}{\mathrm{d} t} \widetilde{\varepsilon}=-C_{\varepsilon 2} \widetilde{\omega} \widetilde{\varepsilon},
\end{aligned}
$$


from an intermediate diamond state, during $\Delta t$. A combination of the two last equations of this system yields $\frac{\mathrm{d}}{\mathrm{d} t} \widetilde{\omega}=-\left(C_{\varepsilon 2}-1\right) \widetilde{\omega}^{2}$, which can be solved analytically.

Proposition 4.26. The following semi-discrete scheme in time

$$
\begin{aligned}
& {\widetilde{Y_{\alpha}^{\prime \prime} \mathbf{u}^{\prime \prime}}}^{(n+1)}=\left(1+\frac{1}{2} C_{1} \widetilde{\omega}^{(n)} \Delta t\right)^{-1}\left(1+\frac{1}{2} C_{\tau} \widetilde{\omega}^{(n)} \Delta t\right)^{-1} \widetilde{Y_{\alpha}^{\prime \prime} \mathbf{u}^{\prime \prime}}, \\
& {\widetilde{Y_{\alpha}^{\prime \prime} Y_{\beta}^{\prime \prime}}}^{(n+1)}=\left(1+C_{\tau o} \widetilde{\omega}^{(n)} \Delta t\right)\left(1+\frac{1}{2} C_{\tau} \widetilde{\omega}^{(n)} \Delta t\right)^{-2} \widetilde{Y_{\alpha}^{\prime \prime} Y_{\beta}^{\prime \prime}}, \\
& {\widetilde{\mathbf{u}^{\prime \prime} \otimes \mathbf{u}^{\prime \prime}}}^{(n+1)}=\left(\frac{2}{3} \widetilde{\omega}^{(n)} \widetilde{k}^{(n)}\left(C_{1}-1\right) \Delta t \mathbb{I}+\widetilde{\mathbf{u}^{\prime \prime} \otimes \mathbf{u}^{\prime \prime}}{ }^{\diamond}\right)\left(1+\frac{1}{2} C_{1} \widetilde{\omega}^{(n)} \Delta t\right)^{-2}, \\
& \widetilde{\varepsilon}^{(n+1)}=\underbrace{\widetilde{\omega}^{(n)}\left(1+\left(C_{\varepsilon 2}-1\right) \widetilde{\omega}^{(n)} \Delta t\right)^{-1}}_{=\widetilde{\omega}^{(n+1)}} \widetilde{k}^{(n+1)},
\end{aligned}
$$

is realizable, in the sense that $\mathbb{X}^{(n+1)} \geq 0$ and $\widetilde{\varepsilon}^{(n+1)} \geq 0$ if $\mathbb{X}^{\diamond} \geq 0$ and $\widetilde{\varepsilon}^{\diamond} \geq 0$.

Proof. Let us introduce the diagonal tensor $\mathbb{G}_{o}=\left[\begin{array}{cc}G_{u u} \mathbb{I} & \mathbf{0} \\ \mathbf{0} & G_{d}\end{array}\right]$, which is obtained from the matrix $\mathbb{G}$, where production gradients have been discarded (following the splitting). In order to obtain the discrete realizability, the terms related to $\mathbb{G}_{O}$ have been considered implicit in equations (4.30)-(4.32), with a realizability correction in $\mathcal{O}\left(\Delta t^{2}\right)$. The semi-discretization in time can be put in the following tensorial form

$$
\left[\mathbb{I}-\Delta t \mathbb{G}_{o}^{(n)}\right] \mathbb{X}^{(n+1)}\left[\mathbb{I}-\Delta t \mathbb{G}_{o}^{(n)}\right]=\mathbb{X}^{\diamond}+\Delta t \mathbb{H}^{\diamond} \mathbb{H}^{\diamond t},
$$

where $\mathbb{G}_{o}^{(n)}=\left[\begin{array}{ll}G_{u u}^{(n)} \mathbb{I} & 0 \\ 0 & G_{d}^{(n)}\end{array}\right], G_{u u}^{(n)}=-\frac{C_{1}}{2} \widetilde{\omega}^{(n)}$, and $G_{d}^{(n)}=-\frac{C_{\tau}}{2} \widetilde{\omega}^{(n)}$. The tensor $\left[\mathbb{I}-\Delta t \mathbb{G}_{o}\right]$ is diagonal with strictly positive diagonal components, thus inversible. We obtain

$$
\mathbb{X}^{(n+1)}=\left[\mathbb{I}-\Delta t \mathbb{G}_{o}^{(n)}\right]^{-1}\left[\mathbb{X}^{\diamond}+\Delta t \mathbb{H}^{\diamond} \mathbb{H}^{\diamond t}\right]\left[\mathbb{I}-\Delta t \mathbb{G}_{o}^{(n)}\right]^{-1} \geq 0 .
$$

Remark 4.27. Discrete realizability can straightforwadly be deduced from Proposition 4.26, where cell indices have been dropped, for the sake of clarity. This proposition does not introduce additional CFL constraint. An accuracy constraint arises at all localizations in the resolution domain, which can be expressed as $\max _{j}\left(\widetilde{\omega}_{j}^{(n)} \Delta t\right) \ll 1$.

\subsection{Summary of the numerical analysis}

Under the following stability restriction on the time step, $\max _{j}\left(\sqrt{\left.\widetilde{u_{x}^{\prime \prime 2}}\right|_{j} ^{(n)}} \frac{\Delta t}{\Delta x_{j}}\right) \leq 1$, we propose a secondorder scheme in space for the hyperbolic part of the model (3.3)-(3.8). It is based on a splitting procedure, that isolates the dissipation and redistribution terms. Let us summarize the full algorithm.

The first step of the splitting procedure computes an intermediate diamond state. At first order, this intermediate state is computed with the discrete equations (4.12), (4.13) and (4.19), together with the first-order solution of the nodal solver, equations (4.7) and (4.8). At second order, the diamond state is computed according to the detailed algorithm presented in Section 4.10, steps (1) to (4). The latter algorithm relies on Proposition 4.24, which allow the separate computation of the diamond state, first for the variables $\mathcal{U}_{\alpha}$ and $\widetilde{Y}_{\alpha, i}$ (steps (1) to (3)), then for the covariance $\widetilde{Y_{\alpha}^{\prime \prime} Y_{\beta}^{\prime \prime}}$ (step (4)), while preserving discrete realizability. 
The second step of the splitting computes a final state, from the intermediate diamond state, owing to the discrete equations (4.30)-(4.33).

The numerical solution satisfies, for arbitrary geometry, the following properties, at the discrete level:

- entropy stability,

- preservation of stationary states,

- realizability,

- conservativity of the mass fraction and material constituent equations,

- mass conservation.

Moreover, the stability restriction on the time step is not stringent and ensures that the time step does not vanish.

\section{NumERICAL TESTS}

We propose four different test cases, three of them having analytical solutions. They highlight the variety of possible regimes that the RSM under study is able to describe. The first one focuses on wave-like behaviour in an acoustic-like regime, while the second focuses on transitory discontinuous solutions, occuring typically at the initialization stage of the model. The third test case deals with the important self-similar regime. The fourth test case deals with the transient demixing regime. In the first three cases, we investigate the convergence rate, showing the benefit of the second-order scheme. For the sake of clarity, a mixture of only two materials, having index $\alpha$ and $\beta$, will be considered in each test. The mass fractions and fluxes of material $\beta$ is easily deduced from that of material $\alpha$, hence we present results only on material $\alpha$. The mean velocity $\widetilde{\mathbf{U}}$ is assumed to be zero.

\subsection{Test case in the acoustic-like regime}

In the regime considered here, the settings are compatible with the Von Neumann analysis of Section 4.8, with periodic boundary conditions. The stability analysis is confirmed numerically for the variety of wavenumbers, and time discretizations shown in Figures 2 and 3. Let us now investigate the convergence properties and the behaviour of the a posteriori procedure. We recall that the diffusion and dissipation terms are discarded. The density is set to one, and the Reynolds tensor is chosen as a constant, in a planar geometry. Thus the "acoustic" speed reduces to $\lambda=\sqrt{u_{x}^{\prime \prime 2}}$. The initial mass fraction fluxes and covariances are set to zero, while the initial mass fraction is chosen as

$$
\widetilde{Y}_{\alpha}^{o}(x)=\frac{1}{2}\left[1+\sin \left(2 k \pi \frac{x}{|\Omega|}\right)\right] .
$$

Here, the spatial domain, $\Omega=[-10 ; 10]$, is chosen periodic, with length $|\Omega|=20$. It is splitted uniformly in $N$ cells, so that $\left|\Omega_{j}\right| \equiv \Delta x$. The wavenumber is set to $k=20$. The analytical solution reads

$$
\begin{aligned}
\tilde{Y}_{\alpha}(t, x) & =\frac{1}{2}\left[\widetilde{Y}_{\alpha}^{o}(x+\lambda t)+\widetilde{Y}_{\alpha}^{o}(x-\lambda t)\right], \\
\widetilde{Y_{\alpha}^{\prime \prime} u_{x}^{\prime \prime}}(t, x) & =\frac{1}{2} \lambda\left[\widetilde{Y}_{\alpha}^{o}(x+\lambda t)-\widetilde{Y}_{\alpha}^{o}(x-\lambda t)\right], \\
\widetilde{Y_{\alpha}^{\prime \prime 2}}(t, x) & =\widetilde{Y_{\alpha}^{\prime \prime} u_{x}^{\prime \prime}}(t, x)^{2} / \lambda^{2} .
\end{aligned}
$$

For the purpose of convergence study, the spatial resolution varies in the range $\Delta x \in\left[10^{-4} ;|\Omega|\right]$, while the time step is fixed at $\Delta t=10^{-4}$. Hence, the stability condition is satisfied for any value of $\Delta x$ considered.

We show in Figure 4, on the right-hand side, the convergence curves. The logarithm of the $L_{1}$-error is plotted against $\log N$, for each variables, and for both the first-order (solid curves) and second-order (dashed curves) schemes. The convergence rates are given in Table 2. As expected, the second-order scheme is second-order 

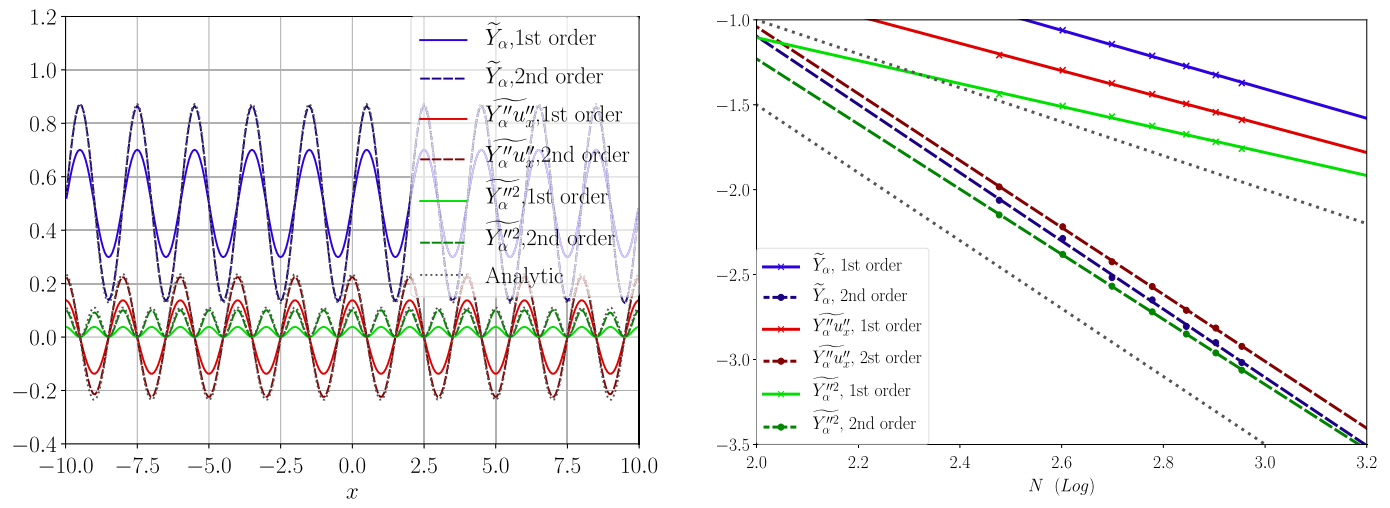

FiguRE 4. Left-hand panel: solution of the test case in the acoustic-like regime, at $t=2.5$ for $\Delta x=2 / 30$, for the first-order (solid curves) and the second-order (dashed curves) scheme. The analytical solution is represented by the dotted curves. The mass fractions (blue), mass fraction fluxes (red) and mass fraction covariances (green) are plotted. Right-hand panel: logarithm of the $L_{1}$-error against $\log N$. The dotted lines correspond to the targeted convergence rates, 1 and 2. The symbols correspond to the different mesh refinements. The associated best-fit lines are also shown.

TABLE 2. Test case in the acoustic-like regime: convergence rates.

\begin{tabular}{lll}
\hline \hline & 1st order & 2nd order \\
\hline$\widetilde{\tilde{Y}_{\alpha}}$ & 0.863 & 2.010 \\
$\widetilde{Y_{\alpha}^{\prime \prime} u_{x}^{\prime \prime}}$ & 0.804 & 1.971 \\
$\widetilde{Y_{\alpha}^{\prime \prime} Y_{\beta}^{\prime \prime}}$ & 0.677 & 1.917 \\
\hline
\end{tabular}

convergent. The convergence rate of the first-order scheme is slightly smaller for the mass fraction covariances. This is due to the fact that these variables depend nonlinearly on the numerical dissipation of both the mass fractions and their fluxes.

As an illustration, Figure 4 also shows, on the left-hand panel, the numerical and analytical solutions at time $t=2.5$ with $N=300$ and $\Delta t=10^{-4}$. We clearly observe that the second-order scheme is closer to the analytical solution, because it is less damped than the first-order scheme. For larger time steps, with the same spatial resolution, the improvement from the first-order to the second-order scheme can still be observed at $\mu=1 / 2$, where $\mu=\sqrt{u^{\prime 2}} \Delta t / \Delta x$. Above $\mu=0.7$, the second-order solution is degraded to first order, by the a posteriori entropy constraint, which we consider acceptable.

\subsection{Initialization test case: the Riemann problem}

Let us now investigate the behaviour of our scheme with respect to the discontinuous, analytical solutions of a Riemann problem. We wish, in particular, to evaluate the treatment of the non-conservative terms by our scheme. Diffusion and dissipation terms are again discarded, while the initial mass fractions are chosen as Heaviside functions. An initial constant heterogeneity level is prescribed via the mass fraction covariances. The analytical solution is resumed in Table 4, where the initial condition is chosen realizable. A mixing zone evolves from the initial location of the mass fraction discontinuity, and expands at a constant speed, equal to the diagonal elements of the Reynolds tensor. For each material, two constituents have been considered, having initial linear profiles. The constituent ratio was initially $99 / 1 \%$ at the domain edges, for the material with index $\alpha$. 
TABLE 3. Convergence rates corresponding to Figure 5, for the Riemann problem.

\begin{tabular}{lll}
\hline \hline & First-order scheme & Second-order scheme \\
\hline$\widetilde{Y}_{\alpha}$ & 0.497 & 0.685 \\
$\widetilde{Y_{\alpha} u_{x}^{\prime \prime}}$ & 0.497 & 0.685 \\
$\widetilde{Y_{\alpha}^{\prime \prime 2}}$ & 0.5 & 0.685 \\
\hline
\end{tabular}

TABLE 4. The Riemann problem: initial condition and analytical solution.

\begin{tabular}{lllllll}
\hline \hline & $\widetilde{Y}_{\alpha}$ & $\widetilde{Y_{\alpha}^{\prime \prime} u_{x}^{\prime \prime}}$ & $\widetilde{Y_{\alpha}^{\prime \prime 2}}$ & $\widetilde{u_{x}^{\prime \prime 2}}=\widetilde{u_{y}^{\prime \prime 2}}=\widetilde{u_{z}^{\prime \prime 2}}=\widetilde{k} / 3$ & $\bar{\rho}$ & $\widetilde{\varepsilon}$ \\
\hline$x<-\sqrt{\widetilde{u_{x}^{\prime \prime 2}}} t$ & 1 & 0 & 1.1 & 0.5 & 1 & 0 \\
$-\sqrt{\widetilde{u_{x}^{\prime \prime 2}} t}<x<\sqrt{\widetilde{u_{x}^{\prime \prime 2}} t}$ & 0.5 & $1 /(2 \sqrt{2})$ & 1.35 & 0.5 & 1 & 0 \\
$x>\sqrt{\widetilde{u_{x}^{\prime \prime 2}}} t$ & 0 & 0 & 1.1 & 0.5 & 1 & 0 \\
\hline
\end{tabular}

The constituent ratio for the material with index $\beta$ was initially chosen $70 / 30 \%$ at the domain edges. The resolution domain, $\Omega=[-10 ; 10]$, is splitted uniformly in $N$ cells, so that $\left|\Omega_{j}\right| \equiv \Delta x$. The spatial resolution varies in the range $\Delta x \in[0.02 ;|\Omega|]$, while the time step is uniquely set at $\Delta t=10^{-3}$, to satisfy the stability conditions at the highest spatial resolution, that corresponds to $\Delta x=0.02$.

Figure 5 (with Table 3), in the right-hand panel, and Figure 6, show good convergence results. The logarithm of the $L_{1}$-error is plotted against $\log N$, for each variable, for both the first-order (solid curves) and secondorder (dashed curves) scheme. As expected, see [12,27], the first-order scheme converges with a $1 / 2$ rate. The high-order scheme proves to converge at a $2 / 3$ rate. These convergence rates are maintained for the material constituents, despite the fact that the high-order correction of the scheme only occurs in the nodal solver.

On the left-hand panel of Figure 5, the numerical and analytical solutions are plotted at $t=10$ with a resolution of $N=500$ cells, with $\Delta t=10^{-3}$. We do not observe any pathology due to the non-conservative product, which would occur at the initial discontinuity position for a standard Godunov scheme applied for the mass fraction covariances. We again observe the improvement of the second-order over the first-order scheme.

Note that the second-order to first-order degradation by the a posteriori loop is activated at the discontinuities only, whatever the space and time resolution.

\subsection{Test case in the self-similar regime}

We finally propose a test case where diffusion and dissipation terms are considered. The dissipation and diffusion constants are given in Table 5 . We make use of the analytical self-similar solution of Section 3.2.2, with $\widetilde{k}_{o}=1$ and $\widetilde{\varepsilon}_{o}=1$, to obtain a reference solution.

At this point, let us give a few comments about the discretization of the tensorial diffusion operator, which can be written component-wise, under the generic form (in the present conditions, assuming constant density)

$$
\frac{\partial}{\partial t} \varphi-\partial_{x}\left(\nu_{T} \partial_{x} \varphi\right)=0
$$

where $\nu_{T}$ is the turbulent diffusivity. It is splitted from the hyperbolic part, with a Strang splitting, to get a global second-order time discretization if a second-order global accuracy is required. A centered discretization in space is used. The semi-discrete scheme is

$$
\left.\frac{\partial}{\partial t} \varphi\right|_{j}-\left(\left.\nu_{T}\right|_{j+1 / 2}\left(\varphi_{j+1}-\varphi_{j}\right) / \Delta x_{j+1 / 2}-\left.\nu_{T}\right|_{j-1 / 2}\left(\varphi_{j}-\varphi_{j-1}\right) / \Delta x_{j-1 / 2}\right) / \Delta x_{j}=0
$$



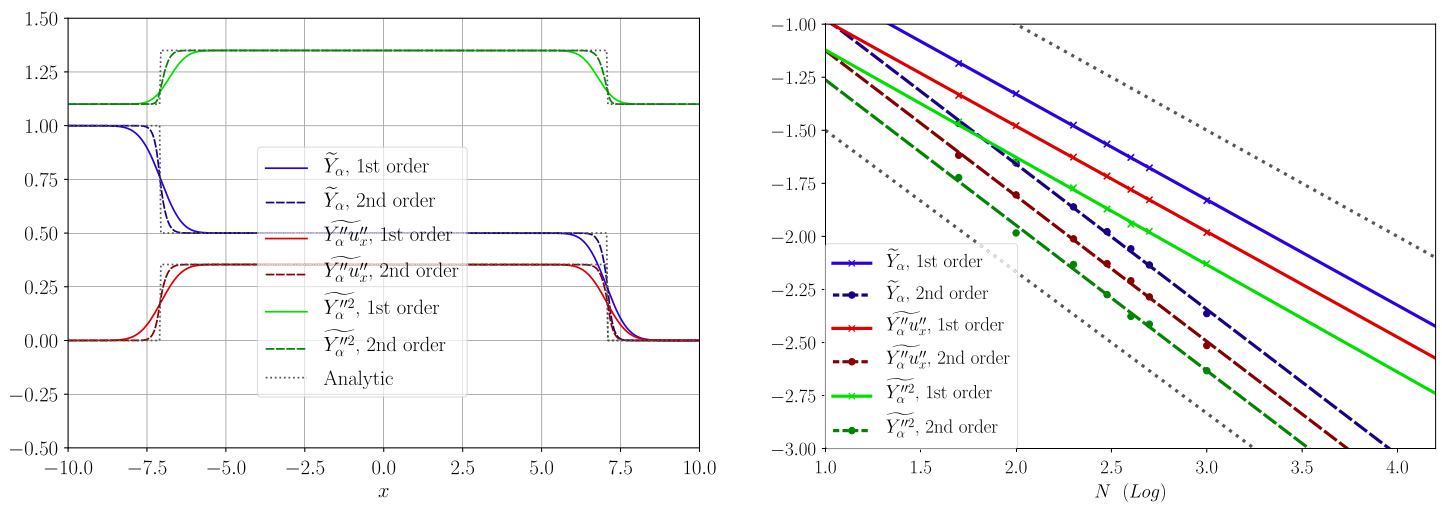

FiguRE 5. Left-hand panel: solution of the Riemann problem, as defined in Table 4 (dotted curves), and numerical solution from the first-order (solid curves) and second-order (dashed curves) schemes. The results for the mass fractions (blue), mass fraction fluxes (red) and mass fraction covariances (green) are plotted at $t=5$ for $N=500$ cells. Right-hand panel: logarithm of the $L_{1}$-error against $\log N$. The dotted lines correspond to the observed convergence rates, $1 / 2$ and $2 / 3$ (Table 3 ). The symbols correspond to the different mesh refinements. The associated best-fit lines are also shown.

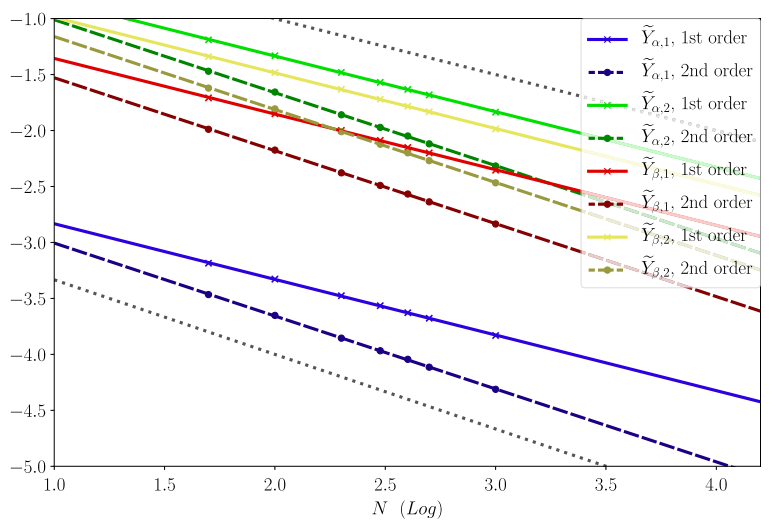

FiguRE 6. Riemann problem: convergence study for the material constituents. Logarithm of the $L_{1}$-error against $\log N$. The dotted lines correspond to a $1 / 2$ and $2 / 3$ slope, close to the observed convergence rates 0.497 and 0.65 , for the first-order and the second-order schemes, respectively. The symbols correspond to the different mesh refinements. The associated best-fit lines are also shown.

with the arithmetic average $\left.\nu_{T}\right|_{j+1 / 2}=\left(\left.\Delta x_{j+1} \nu_{T}\right|_{j}+\left.\Delta x_{j} \nu_{T}\right|_{j+1}\right) /\left(\Delta x_{j}+\Delta x_{j+1}\right)$, and the definition $\Delta x_{j+1 / 2}=$ $\left(\Delta x_{j}+\Delta x_{j+1}\right) / 2$. Using this arithmetic average makes the TMZ support (i.e. the domain of non-zero Reynolds tensor) able to expand, which is not the case if one uses harmonic averaging. The time discretization of the variable $\varphi$ is chosen implicit, while the discretization of the coefficients $\nu_{T}$ are considered explicit.

The resolution domain, $\Omega=[-10 ; 10]$, is splitted uniformly in $N$ cells, so that $\left|\Omega_{j}\right| \equiv \Delta x$. The spatial resolution varies in the range $\Delta x \in[20 / 510 ;|\Omega|]$, while the time step is fixed at $\Delta t=10^{-4}$, for the purpose of a convergence study. Hence, the stability condition is satisfied for any value of $\Delta x$ considered.

Figure 8 (with Table 6) shows the good behaviour of the scheme with respect to the convergence study. The logarithm of the $L_{1}$-error is plotted against $\log N$, for each variable, and for both the first-order (with solid lines) 
TABLE 5. Test case in the self-similar regime: the model constants.

\begin{tabular}{lllll}
\hline \hline$C_{\epsilon 2}$ & $C_{\tau o}$ & $C_{\tau}$ & $C_{1}$ & $C_{\epsilon}$ \\
\hline 1.92 & 1.2 & 1.5 & 0 & 2.5641 \\
\hline
\end{tabular}
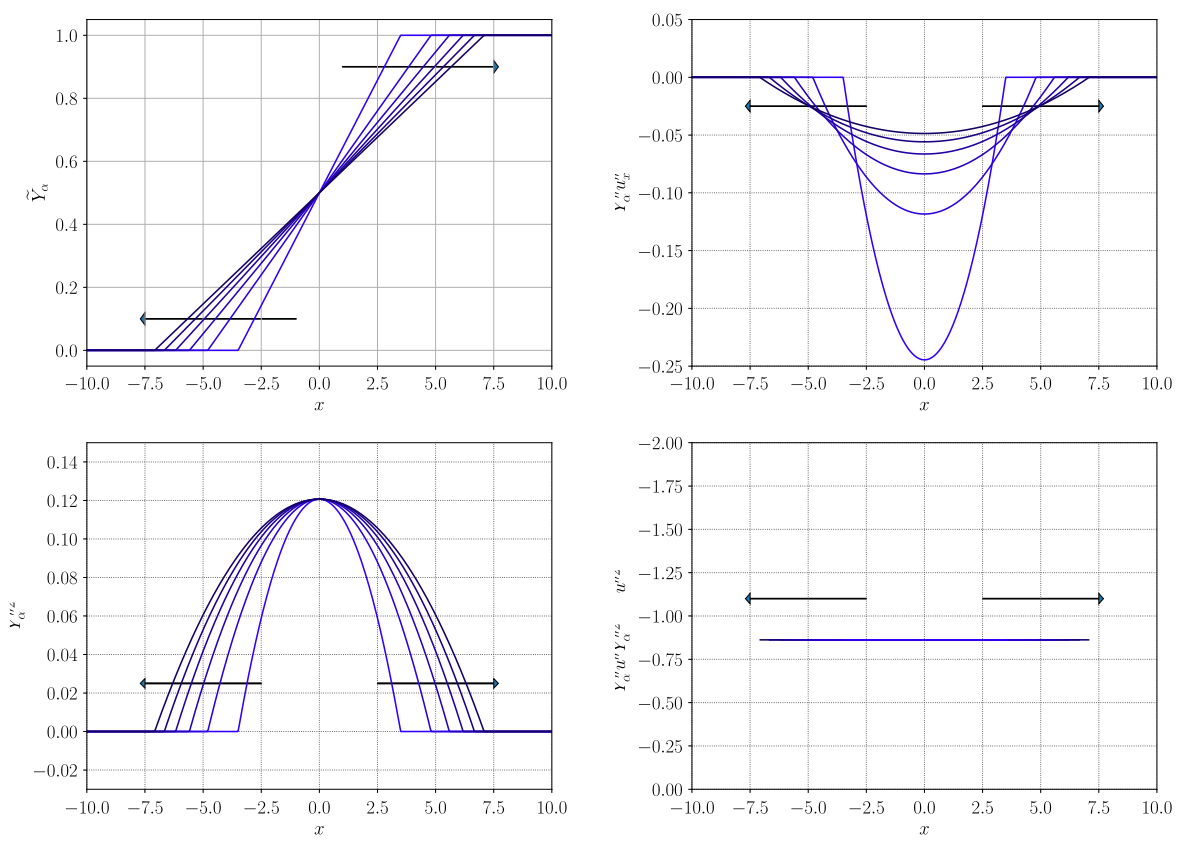

FiguRE 7. Analytical solutions for the self-similar test case. The mass fractions ( upper left-hand panel), mass fraction fluxes (upper right-hand panel), mass fraction covariances (lower left-hand panel) and the slow quantity (lower right-hand panel) are shown. They evolve in time according the arrow directions.

and second-order (with dashed lines) scheme. For the sake of illustration, Figure 7 shows the evolution of the analytical solution of several variables of interest, including the fast computed variables $\widetilde{Y}_{\alpha}, \widetilde{Y_{\alpha}^{\prime \prime} u_{x}^{\prime \prime}}$, and $\widetilde{Y_{\alpha}^{\prime \prime 2}}$, as well as the slow rebuilt variable, $\widetilde{Y_{\alpha}^{\prime \prime} u_{x}^{\prime \prime}} / \sqrt{\widetilde{Y_{\alpha}^{\prime \prime 2}} \widetilde{u_{x}^{\prime \prime 2}}}$. We observe that the second-order scheme brings a subsequent accuracy improvement for all variables, especially for the slow quantity, see Figure 9 for an illustration. This is remarquable, because the analytical reference remains constant across the expanding TMZ, and abruptly falls to zero at its edge, see Figures 7 and 9. We also observe that the a posteriori, second-order to first-order degradation, is not activated, whatever the spatial resolution, and time step ranging from $\Delta t=10^{-4}$ to the maximum stable time step, satisfying $\mu=\sqrt{u^{\prime \prime 2}} \Delta t / \Delta x=1$.

We finally investigate the behaviour of the proposed scheme on non-uniform meshes, again in the case of the self-similar solution. We introduce a geometrical progression across the TMZ, so that boundary cells are 100 times larger than the center cell. Figure 10 gives a comparison between the analytical and numerical solutions at $t=10$, for several variables of interest. As far as mass fractions are concerned, the first-order scheme generates an artificial inflexion point at the TMZ center, while the second-order scheme does not. This illustrates, once again, the beneficial effect of a second-order spatial discretization. The a posteriori correction never activates. We have checked so for time steps ranging from $\Delta t=10^{-4}$ to the maximum stable time step, satisfying $\mu=1$. 

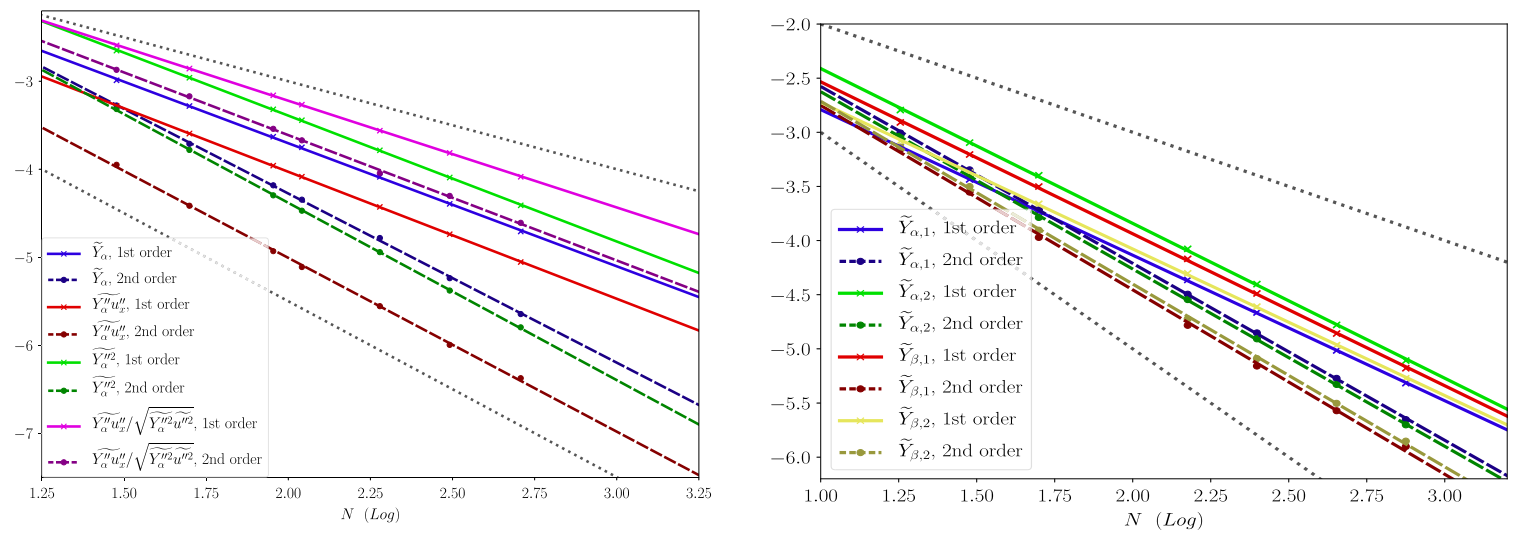

FiguRE 8. Self-similar test case. The logarithm of the $L_{2}$-error is plotted against $\log N$, for a uniform mesh, at $t=10$. Left-hand panel: error curves are shown for the mass fractions (blue), mass fraction fluxes (red), mass fraction covariances (green) and the slow variable (purple). Right-hand panel: error curves are shown for the mass fractions constituents (convergence rates are shown in Table 6). In both panels, the dotted lines correspond to the theoretical convergence rates, 1 and 2 . The symbols correspond to the different mesh refinements. The associated best-fit lines are also shown.

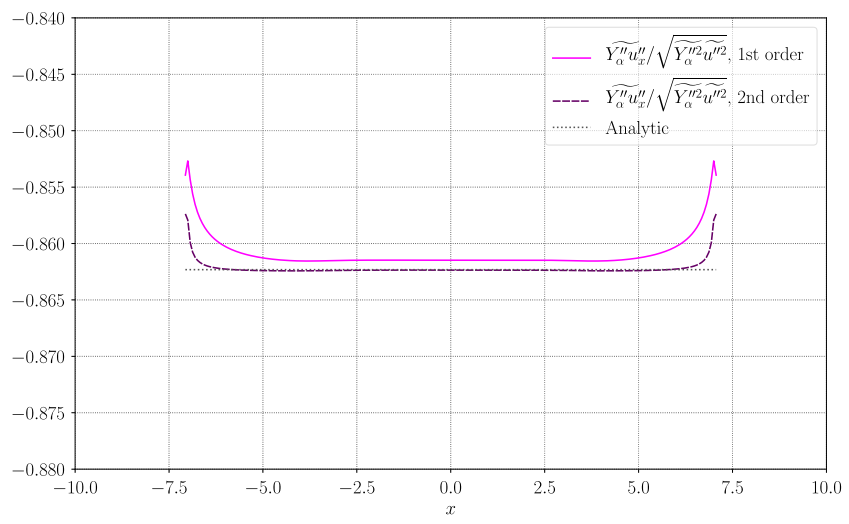

FiguRE 9. Self-similar test case: the analytical solution (dotted line) and the numerical solutions, namely the first-order (solid curve) and second-order (dashed curve) schemes, are shown, at $t=10$, for the slow variable, on a uniform mesh with $N=300$.

\subsection{Test case with transient demixing}

Shock tube experiments can induce transient regimes where demixing occurs, see Figures 4 and 5 in [22]. Indeed, after strong sudden accelerations or shock front crossing, the orientation of mass fraction fluxes can transiently be opposite to the diffusive gradient closed flux of equation (2.14). This so-called counter-gradient situation causes a decrease of the TMZ width, which can be measured as $\mathcal{W}=\int \mathrm{d} x \widetilde{Y}_{\alpha}\left(1-\widetilde{Y}_{\alpha}\right)$. In applications where the chronometry is important (e.g. ICF where only events occurring before maximum compression matter), this non-classical behaviour is important because it introduces a delay in the evolution of the TMZ.

In order to show that our model is able to handle transient demixing, we consider counter-gradient mass fraction flux as an initial condition, and analyse the evolution of $\mathcal{W}$ resulting from the full hyperbolic-parabolic 

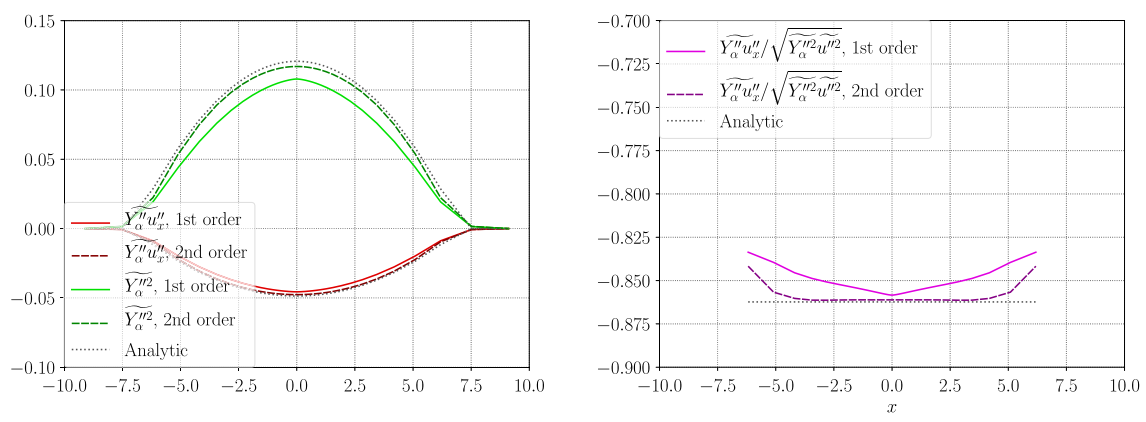

FiguRE 10. Self-similar test case: numerical and analytical solutions on a mesh having a geometric progression, at $t=10$, with $N=50$. The results from the first-order (solid curves), second-order (dashed curves) schemes and the analytical solution (dotted curves) are given for the mass fraction (blue), mass fraction fluxes (red), and mass fraction covariances (green), and the slow variable (purple).

TABLE 6. Convergence rates corresponding to Figure 8, for the self-similar problem.

\begin{tabular}{lll}
\hline \hline & First-order scheme & Second-order scheme \\
\hline$\widetilde{Y}_{\alpha}$ & 1.4 & 1.92 \\
$\widetilde{Y_{\alpha} u_{x}^{\prime \prime}}$ & 1.44 & 1.97 \\
$\widetilde{Y_{\alpha}^{\prime \prime 2}}$ & 1.43 & 2.01 \\
$\widetilde{Y_{\alpha} u_{x}^{\prime \prime}} / \sqrt{\widetilde{Y_{\alpha}^{\prime \prime 2}} \widetilde{u_{x}^{\prime 2}}}$ & 1.21 & 1.42 \\
$\widetilde{Y}_{\alpha, 1}$ & 1.34 & 1.63 \\
$\widetilde{Y}_{\alpha, 2}$ & 1.43 & 1.64 \\
$\widetilde{Y}_{\beta, 1}$ & 1.4 & 1.7 \\
$\widetilde{Y}_{\beta, 2}$ & 1.35 & 1.69 \\
\hline
\end{tabular}

system. We choose the model constants according to Table 5, and propose two computations, at first order, differing only by the initial conditions: the first begins with a diffusive turbulent mass fraction flux (i.e. of the same sign as the first-gradient closed flux in Eq. (2.14)), and the second with an anti-diffusive flux, here taken as the opposite of the previous one. Let us consider one material, denoted by $\alpha$, of a two-material configuration. The chosen initial conditions write

$$
\begin{aligned}
\bar{\rho}(x, t=0) & =1, \\
\widetilde{k}(x, t=0) & =\widetilde{k}_{o} P(x), \\
\widetilde{\varepsilon}(x, t=0) & =\widetilde{\varepsilon}_{o} P(x), \\
\widetilde{Y}_{\alpha}(x, t=0) & =\frac{1}{2}\left(1+\tanh \left(\pi \frac{x}{\Lambda_{o}}\right)\right), \\
\widetilde{Y_{\alpha}^{\prime \prime} u_{x}^{\prime \prime}}(x, t=0) & = \pm\left.\widetilde{Y_{\alpha}^{\prime \prime} u_{x}^{\prime \prime}}\right|_{o} P(x, t), \\
\widetilde{Y_{\alpha}^{\prime \prime 2}}(x, t=0) & =\left.\widetilde{Y_{\alpha}^{\prime \prime 2}}\right|_{o} P(x, t),
\end{aligned}
$$



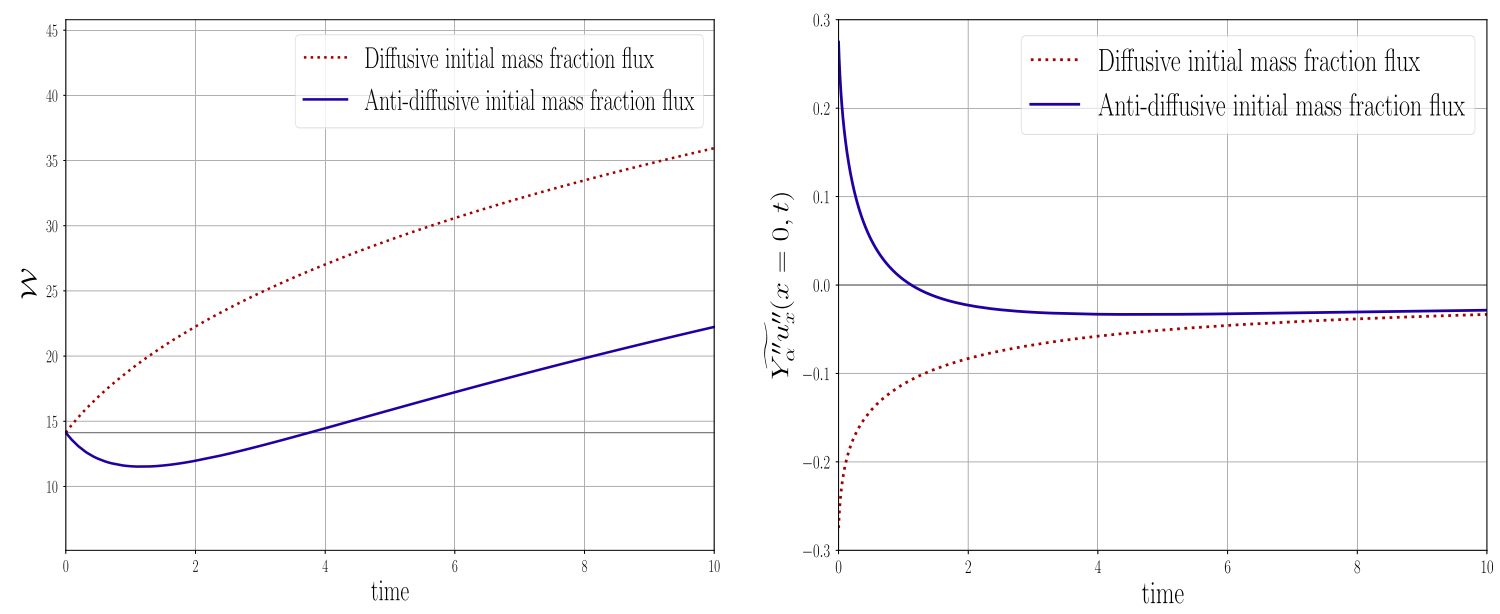

FiguRE 11. Test case with transient demixing: the evolution of $\mathcal{W}=\int \mathrm{d} x \widetilde{Y}_{\alpha}\left(1-\widetilde{Y}_{\alpha}\right)$ and $\widetilde{Y_{\alpha}^{\prime \prime} u_{x}^{\prime \prime}}(x=0, t)$ are shown for two identical initial conditions, only differing by mass fraction fluxes of opposite sign. The initial mass fraction flux, shown in the right-hand panel, is either diffusive (red dotted curve), that is oriented along the first-gradient closed flux of equation (2.14), or anti-diffusive (blue bold curve). In the latter case, a transient demixing induces a delay in the final growth of the TMZ with respect to the former case (left-hand panel).

where

$$
P(x)= \begin{cases}\frac{9}{8} \exp \left(-8 \pi \frac{x^{2}}{\Lambda_{o}^{2}}\right), & \text { if }|\mathrm{x}| \leq \Lambda_{\mathrm{o}} \\ 0, & \text { otherwise }\end{cases}
$$

$\widetilde{k}_{o}=1, \widetilde{\varepsilon}_{o}=1$, while $\Lambda_{o},\left.\widetilde{Y_{\alpha}^{\prime \prime} u_{x}^{\prime \prime}}\right|_{o}$ and $\left.\widetilde{Y_{\alpha}^{\prime \prime 2}}\right|_{o}$ are set so as to satisfy the compatibility relations given in Section 3.2.2. The resolution domain, $\Omega=[-10 ; 10]$, is splitted uniformly in $N=510$ cells. The time step is fixed at $\Delta t=10^{-4}$.

We show, in Figure 11, the evolution of the integral mixing width $\mathcal{W}$, together with the evolution of the turbulent mass fraction flux at the center of the TMZ, from both initial conditions. As expected, a transient demixing is observed for the counter-gradient (anti-diffusive) configuration: the TMZ extent is decreasing for small times, see Figure 11, left-hand panel. Next, due to turbulent production related to the gradient of the mean mass fraction, second term in equation (2.10), the diffusive nature of the turbulent flux is restored with time and both computations should tend to a similar asymptotic state, as made clear by Figure 11, right-hand panel. Since the turbulent flux drives the width of the TMZ, the latter reaches its minimal extent at the time when the sign of the mass fraction flux changes. This phenomenology cannot be accounted for when only diffusive closures are used.

\section{Conclusion}

The mathematical analysis of Reynolds Stress Models (RSM) dedicated to compressible turbulence is delicate: the wave-structure of wide-purpose RSMs, such as BHR [2] and GSG [19,20], is unknown, while they embed, due to the Reynolds and Favre averaging procedure, non-conservative products. We have proposed, in this article, a mathematical and numerical analysis of these models, and focused on a particular extension of them, though representative of a specific limit regime. This extension has been studied independently, but it can be used in the frame of a splitting of the comprehensive RSMs. We have justified, in terms of stability and accuracy, 
the use of an approximate, Godunov-type scheme, in 1D curvilinear coordinates. A broadband set of test-cases has been presented, that aims at covering all the identified relevant regimes. Hence, this work contributes to the mathematical and numerical analysis of RSMs, which deserves better understanding.

The multi-D extension of stable, high-order, and realizable 1D RSM discretization is a natural development, but it is not trivial. The proposed 1D scheme is a good candidate. It can indeed be interpreted as a scheme relying on nodal solvers. In an unstructured multi-D context, nodal solvers indeed enjoy a larger stencil than face-based solvers, encompassing neighbour meshes via nodes. In this respect, the resulting schemes can be considered closer to the genuine multi-D Riemann problem, especially for meshes having a large aspect ratio. We have paved the way towards a multi-D adaptation of the proposed scheme, into the GLACE or EUCCLHYD framework $[13,25]$, as we intend to show in a future companion paper. In this adaptation, the material mass fraction and their fluxes would play respectively the role of the mean pressure and velocity of the Euler equation, in the acoustic nodal solver. Further, the mathematical entropies would play the role of the Gibbs entropy. We have also introduced an a posteriori limitation at high order, that may also bring an essential, entropy-compliant backstop, in multi-D flows with highly distored meshes.

Among other possible perspectives and applications, this work could be pursued by introducing a molecular inter-diffusion operator for the material mass fraction equations, see [1,42]. Such operator can be stiff, depending on the specific stages of an Inertial Confinement Fusion (ICF) capsule implosion, for instance, as it was shown in [40]. In the numerical analysis point of view, the interplay between the turbulent hyperbolic and the nonturbulent, molecular inter-diffusion part of the model will then have to be considered.

\section{Appendix A. Low storage, high-Order Runge-Kutta schemes}

We recall the low storage Runge-Kutta discretizations of $[17,43]$ for the generic ODE

$$
y^{\prime}=f(y) .
$$

These make use of only Euler explicit schemes or convex combinations between the last stage and the initial stage. Let us denote by $y^{(n)}$ the initial stage, which is an approximation of $y(n \Delta t)$, and $y^{(k)}$, which is the $k$-th stage of the Runge-Kutta scheme. The second-order Runge-Kutta scheme writes

$$
\begin{aligned}
y^{(1)} & =y^{(n)}+\Delta t f\left(y^{(n)}\right), \\
y^{(2)} & =y^{(1)}+\Delta t f\left(y^{(1)}\right), \\
y^{(n+1)} & =\frac{1}{2}\left(y^{n}+y^{(2)}\right),
\end{aligned}
$$

while the third-order and fourth-order schemes write

$$
\begin{aligned}
y^{(1)} & =y^{(n)}+\Delta t f\left(y^{(n)}\right), \\
y^{(2)} & =y^{(1)}+\Delta t f\left(y^{(1)}\right), \\
y^{(3)} & =\frac{3}{4} y^{(n)}+\frac{1}{4} y^{(2)}, \\
y^{(4)} & =y^{(3)}+\Delta t f\left(y^{(3)}\right), \\
y^{(n+1)} & =\frac{1}{3} y^{(n)}+\frac{2}{3} y^{(4)},
\end{aligned}
$$

and

$$
y^{(1)}=y^{n}+\Delta t f\left(y^{(n)}\right)
$$




$$
\begin{aligned}
y^{(2)} & =y^{(1)}+\Delta t f\left(y^{(1)}\right), \\
y^{(3)} & =\frac{6}{7} y^{(n)}+\frac{1}{7} y^{(2)}, \\
y^{(4)} & =y^{(3)}+\Delta t f\left(y^{(3)}\right), \\
y^{(5)} & =\frac{8}{15} y^{(n)}+\frac{7}{15} y^{(4)}, \\
y^{(6)} & =y^{(5)}+\Delta t f\left(y^{(5)}\right), \\
y^{(n+1)} & =\frac{3}{8} y^{(n)}+\frac{5}{8} y^{(6)},
\end{aligned}
$$

respectively.

\section{Appendix B. Supporting Lemma 4.23}

Let $\mathcal{A}$ a be a symmetric matrix

$$
\mathcal{A}=\left(\begin{array}{ccccc}
a & b_{1} & b_{2} & \cdots & b_{N} \\
b_{1} & c_{11} & c_{12} & \cdots & c_{1 N} \\
b_{2} & c_{12} & c_{22} & \cdots & c_{2 N} \\
\vdots & \vdots & \vdots & & \vdots \\
b_{N} & c_{1 N} & c_{2 N} & \cdots & c_{N N}
\end{array}\right)
$$

and $D=\left(d_{i j}\right)_{1 \leq i, j \leq N}$ such as

$$
d_{i j}=\left|\begin{array}{ll}
a & b_{j} \\
b_{j} & c_{i j}
\end{array}\right|=a c_{i j}-b_{i} b_{j}=d_{j i} .
$$

Let us show that if $\mathcal{A} \geq 0$, then $D \geq 0$.

Proof. For any vector $X=\left(x_{1}, x_{2}, \cdots, x_{N}\right)^{t}$ :

$$
X^{t} D X=\sum_{i, j=1}^{N}\left(a c_{i j}-b_{i} b_{j}\right) x_{i} x_{j}=a X^{t} C X-\left(b^{t} X\right)^{2} .
$$

We introduce $Y=\left(y, x_{1}, x_{2}, \cdots, x_{N}\right)^{t}$ a vector of $\mathbb{R}^{N+1}$, where $y$ is an arbitrary scalar. Let us assume that $\mathcal{A}$ is a SDP matrix, thus $Y^{t} \mathcal{A} Y \geq 0$, which can be developed as

$$
Y^{t} \mathcal{A} Y=\left(\begin{array}{ll}
y & X^{t}
\end{array}\right)\left(\begin{array}{ll}
a & b^{t} \\
b & C
\end{array}\right)\left(\begin{array}{c}
y \\
X
\end{array}\right)=a y^{2}+2\left(b^{t} X\right) y+X^{t} C X \geq 0 .
$$

This is a polynomial expression in the $y$-variable. It is always non-negative, so its discriminant is non-positive

$$
\left(b^{t} X\right)^{2}-a X^{t} C X \leq 0,
$$

which can be rewritten as $X^{t} D X \geq 0$. Thus $D \geq 0$.

Acknowledgements. The authors wish to acknowledge Cédric Enaux for discussions about the stationary states of the hyperbolic model in curvilinear geometry, in relation with the stability condition. 


\section{REFERENCES}

[1] P. Arnault, Modeling viscosity and diffusion of plasma for pure elements and multicoponent mixtures from weakly to strongly coupled regimes. High Energy Density Phys. 9 (2013) 711-721.

[2] A. Banerjee, R.A. Gore and M.J. Andrews, Development and validation of a turbulent-mix model for variable-density and compressible flows. Phys. Rev. E 82 (2010) 046309.

[3] G.-I. Barenblatt, Self-similar turbulence propagation from an instantaneous plane source. Nonlinear Dyn. Turbul. (1983) 48-60.

[4] C. Berthon and V. Desveaux, An entropy preserving MOOD scheme for the Euler equations. Int. J. Finite 11 (2014) 39.

[5] C. Berthon and D. Reigner, An approximate nonlinear projection scheme for a combustion model. ESAIM: M2AN 37 (2003) 451-478. DOI: $10.1051 / \mathrm{m} 2 \mathrm{an}: 2003037$

[6] C. Berthon, F. Coquel, J.-M. Hérard and M. Uhlmann, An approximate solution of the Riemann problem for a realizable second-moment turbulent closure. Shock. Waves 11 (2002) 245-269.

[7] F. Bouchut, Nonlinear Stability of Finite Volume Methods for Hyperbolic Conservation Laws. Birkhäuser Basel (2004).

[8] Y. Bury, P. Graumer, S. Jamme and J. Griffond, Turbulent transition of a gaseous mixing zone induced by the RichtmyerMeshkov instability. Phys. Rev. Fluids 5 (2020) 024101.

[9] G. Carré, S. Del Pino, B. Després and E. Labourasse, A cell centered Lagrangian hydrodynamics scheme on general unstructured meshes in arbitrary dimension. J. Comput. Phys. 228 (2009) 5160-5183.

[10] C. Cherfils and A.K. Harrison, Comparison of different statistical models of turbulence by similarity methods. In: Presented at the 1994 ASME Fluids Engineering Summer Meeting (May 1994) 19.

[11] J.-F. Clouet, The Rosseland approximation for radiative transfer problems in heterogeneous media. J. Quant. Spectrom. Radiat. Transfer 58 (1997) 33-43.

[12] F. Delarue and F. Lagoutière, Probabilistic analysis of the upwind scheme for transport equations. Arch. Ration. Mech. Anal. 199 (2011) 229-268.

[13] B. Després and C. Mazeran, Lagrangian gaz dynamics in two dimensions and lagrangian schemes. Arch. Ration. Mech. Anal. 178 (2005) 327-371.

[14] C. Dopazo, Probability density function approach for a turbulent axisymmetric heated jet. Centreline evolution. Phys. Fluids 18 (1975) 397-404.

[15] C. Emako, V. Letizia, N. Petrova, R. Sainct, R. Duclous and O. Soulard, Diffusion limit of the simplified Langevin PDF model in weakly inhomogeneous turbulence. ESAIM: Proc. Surv. 48 (2015) 400-419.

[16] A. Favre, L.S.G. Kovasznay, R. Dumas, J. Gaviglio and M. Coantic, La turbulence en mécanique des fluides: bases théoriques et expérimentales, méthodes statistiques. Gauthier-Villars, Paris (1976).

[17] S. Gottlieb, C.-W. Shu and E. Tadmor, Strong stability-preserving high-order time discretization methods. SIAM Rev. 43 (2001) 89-112.

[18] B.-J. Gréa, The dynamics of the $k-\varepsilon$ mix model toward its self-similar Rayleigh-Taylor solution. J. Turbulence 16 (2015) 184-202.

[19] O. Grégoire, D. Souffland and S. Gauthier, A second-order turbulence model for gaseous mixtures induced by RichtmyerMeshkov instability. J. Turbul. 6 (2005) N29.

[20] J. Griffond and O. Soulard, Evaluation of augmented RSM for interaction of homogeneous turbulent mixture with shock and rarefaction waves. J. Turbul. 15 (2014) 569-595.

[21] J. Griffond, O. Soulard and D. Souffland, A turbulent mixing Reynolds stress model fitted to match linear interaction analysis predictions. Phys. Scr. 2010 (2010) 014059.

[22] J. Griffond, J.-F. Hass, D. Souffland, G. Bouzgarrou, Y. Bury and S. Jamme, Experimental and numerical investigation of the growth of an air/SF6 turbulent mixing zone in a shock tube. J. Fluid Eng. 139 (2017) 091205.

[23] J.O. Hinze, Turbulence, 2nd edition. McGraw-Hill, New York (1975).

[24] K.K. Mackay and J.E. Pino, Modeling gas-shell mixing in icf with separated reactants. Phys. Plasmas 27 (2020) 092704.

[25] P.-H. Maire, Contribution to the numerical modeling of Inertial Confinement Fusion. Habilitation à diriger des recherches, Université Bordeaux I (February 2011).

[26] B. Meltz, Analyse mathématiques et numérique de système hydrodynamique compressible et de photonique en coordonnées polaires. Ph.D. thesis, Université Paris-Saclay (2015).

[27] B. Merlet and J. Vovelle, Error estimate for finite volume scheme. Numer. Math. 106 (2007) 129-155.

[28] B. Morgan, B.Olson, W. Black and J. McFarland, Large-eddy simulation and Reynolds-averaged Navier-Stokes modeling of a reacting Rayleigh-Taylor mixing layer in a spherical geometry. Phys. Rev. E 98 (2018) 033111.

[29] F. Poggi, M.-H. Thorembey and R. Gérard, Velocity measurements in turbulent gaseous mixtures induced by RichtmyerMeshkov instability. Phys. Fluids 10 (1998) 2698-2700.

[30] S.B. Pope, PDF methods for turbulent reactive flows. Prog. Energy Combust. Sci. 11 (1985) 119-192.

[31] S.B. Pope, On the relationship between stochastic Lagrangian models of turbulence and second-moment closures. Phys. Fluids 6 (1994) 973-985.

[32] S.B. Pope, Turbulent Flows. Cambrige University Press (2000).

[33] J.R. Ristorcelli, Exact statistical results for binary mixing and reaction in variable density turbulence. Phys. Fluids 29 (2017) 020705.

[34] R. Schiestel, Méthodes de Modélisation et de Simulation des Ecoulements Turbulents. Hermès/Lavoisier (2006). 
[35] R. Schiestel, Modeling and Simulation of Turbulent Flows. John Wiley \& Sons, Ltd (2008).

[36] D. Souffland, O. Soulard and J. Griffond, Modeling of Reynolds stress models for diffusion fluxes inside shock waves. J. Fluids Eng. 136 (2014) 091102.

[37] O. Soulard, F. Guillois, J. Griffond, V. Sabelnikov and S. Simoëns, Permanence of large eddies in Richtmyer-Meshkov turbulence with a small Atwood number. Phys. Rev. Fluids 3 (2018) 104603.

[38] O. Soulard, F. Guillois, J. Griffond, V. Sabelnikov and S. Simoëns, A two-scale Langevin pdf model for Richtmyer-Meshkov turbulence with a small Atwood number. Phys. D: Nonlinear Phenom. 403 (2020) 132276.

[39] D. Veynante and L. Vervisch, Turbulent combustion modeling. Prog. Energy Combust. Sci. 28 (2002) $193-266$.

[40] G. Viciconte, B.-J. Gréa, F.S. Godefer, P. Arnault and J. Clérouin, Sudden diffusion of turbulent mixing layers in weakly coupled plasmas under compression. Phys. Rev E 100 (2019) 063205.

[41] J. Vides, B. Braconnier, E. Audit, C. Berthon and B. Nkonga, A Godunov-Type solver for the numerical approximation of gravitational flows. Commun. Comput. Phys. 15 (2014) 46-75.

[42] E.L. Vold, A.S. Joglekar, M.I. Ortega, R. Moll, D. Fenn and M. Kim, Plasma viscosity with mass transport in spherical inertial confinement fusion implosion simulations. Phys. Plasmas 22 (2015) 112708.

[43] J.H. Williamson, Low storage Runge-Kutta schemes. J. Comput. Phys. 35 (1980) 48-56.

[44] J.G. Wouchuk and K. Nishihara, Asymptotic growth in the linear Richtmyer-Meshkov instability. Phys. Plasmas 4 (1997) $1028-1038$.

\section{Subscribe to Open (S2O)}

\section{A fair and sustainable open access model}

This journal is currently published in open access under a Subscribe-to-Open model (S2O). S2O is a transformative model that aims to move subscription journals to open access. Open access is the free, immediate, online availability of research articles combined with the rights to use these articles fully in the digital environment. We are thankful to our subscribers and sponsors for making it possible to publish this journal in open access, free of charge for authors.

\section{Please help to maintain this journal in open access!}

Check that your library subscribes to the journal, or make a personal donation to the S2O programme, by contacting subscribers@edpsciences.org

More information, including a list of sponsors and a financial transparency report, available at: https://www. edpsciences.org/en/maths-s2o-programme 